University of Louisville

ThinkIR: The University of Louisville's Institutional Repository

$12-2011$

\title{
Cytotoxicity and cellular response to alpha, beta-unsaturated aldehydes : role of aldehyde dehydrogenases.
}

Ngome Llewellyn Makia

University of Louisville

Follow this and additional works at: https://ir.library.louisville.edu/etd

\section{Recommended Citation}

Makia, Ngome Llewellyn, "Cytotoxicity and cellular response to alpha, beta-unsaturated aldehydes : role of aldehyde dehydrogenases." (2011). Electronic Theses and Dissertations. Paper 892.

https://doi.org/10.18297/etd/892

This Doctoral Dissertation is brought to you for free and open access by ThinkIR: The University of Louisville's Institutional Repository. It has been accepted for inclusion in Electronic Theses and Dissertations by an authorized administrator of ThinkIR: The University of Louisville's Institutional Repository. This title appears here courtesy of the author, who has retained all other copyrights. For more information, please contact thinkir@louisville.edu. 
CYTOTOXICITY AND CELLULAR RESPONSE TO ALPHA, BETAUNSATURATED ALDEHYDES. ROLE OF ALDEHYDE DEHYDROGENASES

By

Ngome Llewellyn Makia

BSc., University of Buea, Cameroon, 2000

MSc., University of Nottingham, England, 2004

\begin{abstract}
A Dissertation
Submitted to the Graduate Faculty of the School of Medicine, University of Louisville in Partial Fulfillment of the requirement for the Degree of
\end{abstract}

Doctor of Philosophy

Department of Biochemistry and Molecular Biology

University of Louisville

Louisville, Kentucky

December 2011 

CYTOTOXICITY AND CELLULAR RESPONSE TO ALPHA, BETA-

UNSATURATED ALDEHYDES, ROLE OF ALDEHYDE DEHYDROGENASES

By

Ngome Llewellyn Makia

B.Sc., University of Buea, Cameroon, 2000

M.Sc., University of Nottingham, England, 2004

A Dissertation Approved on

August 24, 2011

by the following Dissertation Committee:

Russell A. Prough, Ph.D.

Thomas E. Geoghegan, Ph.D.

Barbara J. Clark, Ph.D.

David J. Samuelson, Ph.D.

Daniel J. Conklin, Ph.D.

Kenneth S. Ramos, Ph.D. 


\section{DEDICATION}

This Dissertation is dedicated to my wife,

Mrs Olive Ngalame, for her love and persistent confidence in me. I owe her for unselfishly allowing her aspirations and ambitions to develop with mine.

To my parents, Mr. Elias Mbi Makia (of blessed memory)

and

Mrs. Comfort Okole Makia

who taught me the importance of educational and intellectual pursuits, and for sincerely raising me up with caring and unconditional love. 


\section{ACKNOWLEDGEMENTS}

It is my pleasure to recognize all those who made this thesis a success. I am heartily grateful to my supervisor, Dr Russell A. Prough, for his encouragement, guidance and support from the beginning of my research to the writing of this thesis. His expertise in the area of molecular toxicology and drug metabolism enabled me to develop a better understanding of the subject necessary to complete my dissertation research. Thank you for giving me the opportunity to work in your lab. It was really an honor to work with you. Dr Prough took care of not only my academic needs but also every of my other needs enabling me to settle and excel as a graduate student. I would also like to appreciate Mrs Betty Prough for looking closely at the final version of my thesis for English styles and grammar, and offering useful suggestions for improvement.

I would like to express my gratitude to all my dissertation committee members:

Dr Thomas E. Geoghegan; Dr Daniel J. Conklin, Dr David J. Samuelson, Dr Kenneth S. Ramos and Dr Barbara J. Clark; for their advice, stimulating suggestions and comments on results and experimental designs. They also generated questions to aid in experimental design and development of the project to completion. Finally, they were always ready to provide materials and reagents whenever needed for the completion of the project.

I would also like to express my gratitude to Dr Keith C. Falkner for his support throughout the years with troubleshooting, experimental design, data analysis and 
interpretation of results. He was instrumental during the cloning, expression and purification of the recombinant mouse Aldhs.

Former members of the Prough Lab helped me tremendously in my research project. I am grateful for their support, encouragements and valuable hints to help in the success of my research. Mary Pendleton and Samira Hassan thought me how to perform transient transfections and also assisted me with transfection thereby making my work easier.

My appreciation also goes to the summer students: Zack Simpson, Luz E Guevara and Sara Heintzman for helping with the cloning, expression and characterization of various murine aldehyde dehydrogenases. I wish them well in their future endeavors.

I owe my deepest gratitude to my wife for her persistent confidence in me. She was always there; in my good, bad, low and high moments. I would also like to express my sincere gratitude to my mother-in-law who has been living with us for the past year and half since the birth of our daughter. She made us to have normal life even after having a baby.

I would also like to thank the entire students, staffs and faculty of the Department of Biochemistry and Molecular Biology for their continuous support and encouragements. The Integrated Programs in Biomedical Sciences (IPIBS) for giving me the opportunity to pursue my $\mathrm{PhD}$ in Biochemistry and Molecular Biology. 


\title{
ABSTRACT \\ CYTOTOXICITY AND CELLULAR RESPONSE TO $\alpha, \beta$-UNSATURATED ALDEHYDES. ROLE OF ALDEHYDE DEHYDROGENASES Ngome Makia
}

\begin{abstract}
August 24, 2011
The lipid aldehydes, 4-hydroxy-2-nonenal (4-HNE) and propene-2-al (acrolein) are reactive $\alpha, \beta$-unsaturated aldehydes generated during the peroxidation of lipids and are implicated in the pathogenesis of several oxidative-stress mediated diseases, including steatohepatitis and cancer. We established that mouse liver aldehyde dehydrogenase $1 \mathrm{al}$ (Aldhlal) efficiently metabolizes lipid aldehydes and protects a liver-derived cell line from the toxic effects of these aldehydes. Thus, mechanisms to induce the expression of Aldh1a1 might be a useful rationale for preventing oxidative stress-induced pathologies. We investigate whether well-known electrophiles, such as BHA or acrolein modulate the expression of Aldh1a1 and elucidate the signaling pathway involved. Microarray analyses were performed to examine whether acrolein or BHA up-regulate the expression of genes encoding enzymes involved in antioxidant or electrophile detoxification in mice liver. Mice were administered AIN76A (control) diet, diet containing $0.45 \%$ BHA or 5 $\mathrm{mg} / \mathrm{kg}$ acrolein by gavage for 7 days. The expression of genes encoding several electrophile detoxifying enzymes was specifically elevated, indicating a detoxification response. The elevation of Aldh1al was noticeable, with a 2- to 3-fold increase by both electrophiles. Quantitative real-time PCR analysis also showed $\approx 2.5$-fold and $\approx 3$ -
\end{abstract}


fold induction of Aldh1a1 gene expression by BHA and acrolein, respectively. Livers from BHA- and acrolein-treated mice also showed increased cytosolic Aldh activity compared to control. Acrolein and tert-butylhydroquinone (the metabolized products of BHA) are electrophiles that induce the expression of cytoprotective genes by direct activation of nuclear factor-E2-related factor-2 (Nrf2), activator protein 1 (AP-1) and nuclear factor kappa B (NF- $\mathrm{B}$ ) transcription factors or indirectly by activation of protein kinases, such as MAPKs.

To decipher the signaling pathways involved in Aldh1a1 induction by electrophiles, we analyzed the mRNA levels of Aldh1a1 in the liver of Nrf2+/+ and $\mathrm{Nrf}^{-}$ ${ }^{-}$mice on C57BL6 background exposed to BHA. Mice exposed to BHA showed $\approx 2$-fold increase in mRNA levels of Aldhlal in both Nrf2+/+ and $\mathrm{Nrf}^{-/-}$mice compared to control, indicating that electrophile-induced expression might be independent of $\mathrm{Nrf2}$. However, the mRNA and protein levels of AP-1 and the activity of c-Jun were significantly increased by BHA. We hypothesized that electrophile-induced expression of hepatic Aldh1a1 gene is mediated by activation of AP-1 transcription factor. Transient transfection experiments were conducted in HepG2 cells with Aldh1a1 5'-flanking luciferase reporter constructs. While co-transfection with Nrf2 expression plasmid alone or in the presence of tBHQ had no effect, over-expression of c-Jun/AP-1 resulted in $\approx 4$ fold induction in Aldh1a1 transcriptional activity. Moreover, c-Jun transactivates Aldhlal promoter activity as a homodimer and not c-Jun/c-fos heterodimer. We also established by promoter deletion and mutagenesis analysis that two AP-1 sites at position -758 and -1069 relative to Aldh1a1 transcription start site are responsible for c-Junmediated transactivation of Aldh1a1 luciferase activity. EMSA analysis using biotin- 
labeled probe and super shift with antibodies against c-Jun, c-fos and Nrf2 showed that cJun binds to the proximal AP-1 site at -758 but not at -1069 . The recruitment of c-Jun to this AP-1 site by BHA was confirmed by ChIP experiment, which showed $\approx 10$-fold enrichment to the proximal AP-1 site with c-Jun. These results indicate that electrophiles promote the recruitment of $\mathrm{c}-\mathrm{Jun} / \mathrm{AP}-1$ to the Aldhlal gene promoter, resulting in increased transcription of Aldh1al. 


\section{TABLE OF CONTENTS}

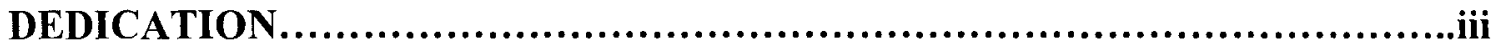

ACKNOWLEGEMENTS...........................................................

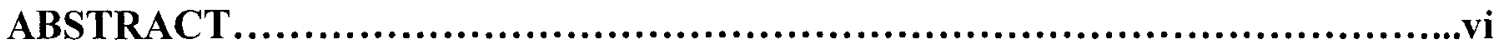

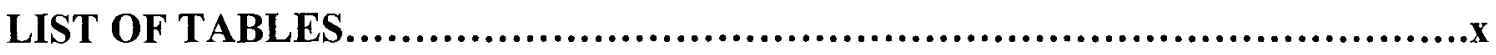

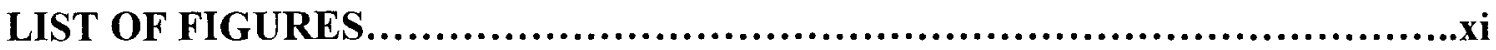

\section{CHAPTER}

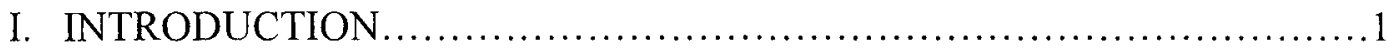

II. MURINE HEPATIC ALDEHYDE DEHYDROGENASE 1A1 IS A MAJOR CONTRIBUTOR TO OXIDATION OF ALDEHYDES

FORMED BY LIPID PEROXIDATION ..................................16

III. MOLECULAR MECHANISMS UNDERLYING TRANSCRIPTIONAL REGULATION OF MOUSE HEPATIC ALDEHYDE DEHYDROGENASE 1A1 BY ELECTROPHILES .............................................49

IV. CHARACTERIZATION OF CIS-REGULATORY ELEMENTS INVOLVED IN REDOX-PROTECTIVE TRANSCRIPTION OF ALDH1A1 GENE ...................................................... 99

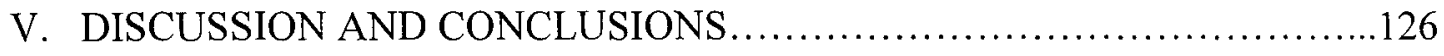

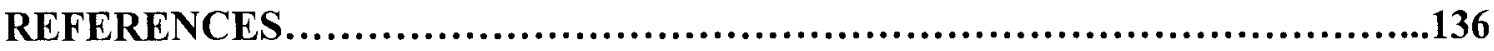

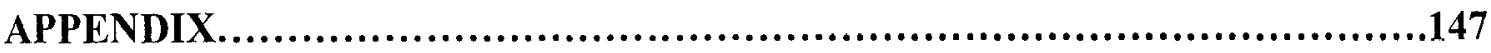

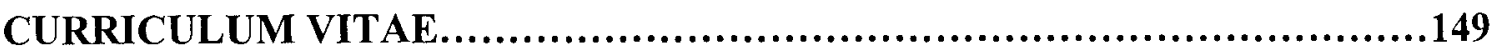




\section{LIST OF TABLES}

TABLE

PAGE

Table 1. Kinetic Properties of recombinant mouse Aldhs........................31

Table 2. Effects of Nrf2 genotype on changes in mRNA levels of antioxidant

and electrophile detoxification genes in response to dietary BHA........67 


\section{LIST OF FIGURES}

FIGURE

PAGE

1. The basic leucine zipper (bZIP) family proteins and their binding partners.

2. SDS-PAGE analyses of purified recombinant mouse Aldh.....

3. Endogenous levels of aldehyde dehydrogenase gene expression in mouse liver and mouse hepatoma cells...

4. Hepa-1c1c7 cells with knockdown of Aldh1al gene expression by stealth specific siRNA are highly sensitive to acrolein-induced cell death compared to control....

5. The levels of acrolein-protein adducts in response to acrolein treatment are increased in Hepa-1c1c7 cells with knockdown of Aldh1al gene compared to control siRNA transfected cells.

6. Transient knockdown of Aldhlal gene expression sensitizes Hepa-1c1c 7 cells to acrolein-induced caspase 3 activation and apoptosis.

7. QRT-PCR analysis of electrophile detoxification gene expression in the liver of mice treated with acrolein, BHA or SFN.

8. Acrolein induces the mRNA levels of cytoprotective genes encoding antioxidant and electrophile-detoxification enzymes in Hepa-1clc7.

9. Increased liver cytosolic ALDH activity by BHA or acrolein in mice.

10. Effect of BHA on $c$-jun and $c$-fos gene expression in mice liver.

11. Effect of acrolein on $c$-jun and $c$-fos transcriptional activity in mice liver and hepalc1c7 cells

12. The Aldh1al transcriptional activity is significantly increased by acrolein...73

13. Western blots indicating over-expression of c-Jun, JunD and $\mathrm{Nrf} 2$ in HepG 2 cells 
14. The Aldh1a1 promoter luciferase activity is not activated by Nrf2 ..........76

15. The Aldh1a1 promoter activity is transactivated by $\mathrm{c}-\mathrm{Jun}$ and $\mathrm{c}-\mathrm{Fos} \ldots \ldots \ldots . . .78$

16. Inhibition of c-Jun mediated transactivation of Aldh1al-luciferase activity by c-Jun dominant negative (TAM67) protein.

17. Effect of Jun family proteins on transactivation of Aldh1 a1-luciferase activity.

18. Over-expression of Jun family proteins in Hepa-1c1c7 increases the endogenous protein levels of Aldh1a1....

19. The effect of heterodimerization between c-jun and fos family proteins (c-fos, Fra-1 and Fra-2) on Aldh1a1 transcriptional activity...

20. Induction of Aldhla1 transcription is mediated by c-Jun homodimer and not by c-Jun/c-Fos heterodimer

21. There is no cross-talk between c-Jun and Nrf2 in tranactivation of Aldh1a1 promoter activity.

22. Aldh1al promoter activity is not responsive to activation of NF- $\mathrm{KB}$ signaling pathway by $\mathrm{TNF} \alpha$.

23. The role of MAPKs in electrophile-mediated transactivation of Aldhlal promoter activity.

24. The nucleotide sequence of mouse Aldhlal promoter depicting potential AP-1, Nrf2 (ARE) and NF-kB binding sites as well as other putative transcription factor $(\mathrm{TF})$ binding sites.

25. Deletion analysis to identify the AP-1-like responsive element in the proximal promoter Aldh1a1 gene.

26. Mutagenesis of the putative AP-1-like elements located at -1069 bp (C site) and $-758 \mathrm{bp}$ (B site) on Aldhlal promoter activity

27. Western blots indicating over-expression of c-Jun in the nuclear extracts of HepG2 cells.

28. EMSA analysis indicates the formation of nuclear protein complex with the AP-1-like element at site B (-758) but not with the AP-1-like element at site C (-1069).... 
29. The nuclear protein complexes with the AP-1-like element at site B $(-758)$ was competed by excess unlabeled wild type probe but not probe with mutation in the AP-1-like element.

30. EMSA analysis indicates no protein binding to the AP-1 site C $(-1069)$ at higher concentrations of nuclear extracts

31. Super shift analysis confirms that a protein in the nuclear extract that binds to the AP-1 site B is c-Jun and not c-fos or Nrf2

32. ChIP analysis to examine in vivo binding of $\mathrm{c}-\mathrm{Jun}$ and $\mathrm{c}-\mathrm{fos}$ to the AP-1 at sites B and C...

33. Proposed schematic model for the activation of Aldhlal gene by electrophiles 


\section{CHAPTER I}

\section{INTRODUCTION}

\section{Lipid and Environmental Aldehydes}

Propene-2-al (acrolein) is a highly reactive $\alpha, \beta$-unsaturated aldehyde ubiquitously present as an environmental pollutant in cigarette smoke, vehicle exhaust emission, drinking water and in effluents from industrial plants. It is produced by a variety of natural and synthetic processes that include the incomplete combustion of organic materials, such as fuels, plastic materials, wood, food, frying oils and tobacco (Stevens and Maier, 2008). The amount of acrolein in a single puff of cigarette smoke is estimated to range between 1.6 -22 $\mu \mathrm{g} /$ cigarette depending on the carbon filter of the cigarette (Thweatt et al., 2007). Acrolein is found at a concentration of 0.04 to $0.08 \mathrm{ppm}$ in ambient air (Beauchamp, Jr. et al., 1985). However, acrolein levels can be as high as 90 ppm in cigarette smoke. Inhalation studies demonstrate that the lethal dose of acrolein in mice is a 10 min exposure of 175 ppm (Beauchamp, Jr. et al., 1985). The main endogenous sources of acrolein are the biotransformation of allyl alcohol, allylamine, spermine, spermidine and the widely used anticancer drug cyclophosphamide. Acrolein is

a major end product formed from the peroxidation of the $\omega-6$ polyunsaturated fatty acids, such as linoleic acid and arachidonic acid along with 4-hydroxynonenal (4-HNE), crotonaldehyde, malondialdehyde (MDA), hexanal and trans-2-hexenal 
(Uchida K et al., 1998). Thus, humans are constantly exposed to acrolein and related aldehydes in the environment. It is estimated that the maximum daily human consumption of unsaturated aldehydes is $\approx 5 \mathrm{mg} / \mathrm{kg}$, while the total aldehyde consumption has been suggested to $b e \approx 7 \mathrm{mg} / \mathrm{kg}$ (Wang et al., 2008;Conklin et al., 2010).

\section{Mechanism of cytotoxicity of $\alpha, \beta$-unsaturated aldehydes}

The $\alpha, \beta$-unsaturated aldehydes, acrolein and $\mathrm{HNE}$, are highly reactive electrophiles and can undergo Michael addition reaction with nucleophilic groups on proteins and DNA, forming stable covalent adducts (Esterbauer, 1993). These aldehydes form adducts with proteins by reacting with cysteine sulfhydryl group, histidine imidazole group and lysine $\varepsilon$-amino group, resulting in alteration of several cellular processes. Enzymes containing amino and sulfhydryl groups in their active site can be inhibited by reactive aldehydes. For example, high levels of acrolein or HNE inhibit aldehyde dehydrogenases, cytochrome P450, GST $m u$ and $p i$ and the glycolytic enzymes, glyceraldehyde-3 phosphate and glucose- 6 phosphate dehydrogenase (Berhane and Mannervik, 1990;Kuo et al., 1997;Silva and O'Brien, 1989;Szweda et al., 1993;Uchida and Stadtman, 1993). The conjugation of $\alpha, \beta$-unsaturated aldehyde with reduced glutathione (GSH) can induce oxidative stress by depleting cells of their natural antioxidant, glutathione. HNE and acrolein are known to induce apoptotic and necrotic cell death by activation of death signaling pathways involving caspases and the mitochondrial permeability transition pore (mMTP) (Burcham and Fontaine, 2001).

Reactive aldehydes are implicated in the pathogenesis of a number of inflammatory diseases in humans. The accumulation of reactive aldehydes in the liver 
can promote the influx of inflammatory cells by stimulating the production of the proinflammatory cytokines, TNF $\alpha$, thereby amplifying inflammatory response (Browning and Horton, 2004;Esterbauer et al., 1991). In addition, reactive aldehydes are also known to activate hepatic stellate cells leading to collagen deposition (Browning and Horton, 2004), suggesting that lipid aldehydes are implicated in the transition from fatty liver (steatosis) to steatohepatitis and other end stage liver diseases, such as liver fibrosis and non-alcoholic steatohepatitis (NASH). HNE and acrolein have also been linked to the pathogenesis of atherosclerosis (Uchida et al., 1994). The low-density lipoprotein (LDL) becomes more atherogenic when modified by acrolein and HNE triggering the uptake of LDL by scavenger receptors located on vascular smooth muscle cells (Uchida et al., 1994;Uchida et al., 1998;Uchida, 2000). Thus, high levels of 4HNE- and acroleinmodified proteins have been detected in atherosclerotic lesions (Uchida et al., 1994;Uchida et al., 1998). Cytotoxic aldehydes play a major role in the progression of myocardial ischemia reperfusion injury by activation of the mMTP (Eaton et al., 1999). The opening of the mMTP is considered a primary event in necrotic cell death in myocardial ischemia-reperfusion injury. Thus, a small-molecule activator of ALDH2 (Alda-1), when administered to rats before an ischemic event, reduced infarct size by $60 \%$, most likely through its inhibitory effect on the formation of protein adducts by electrophilic aldehydes (Chen et al., 2008). Therefore, the metabolism of these toxic compounds to less reactive and excretable compounds is of great importance in ameliorating lipid aldehydes induced human diseases.

\section{The Aldehyde Dehydrogenase (ALDH) genes}


Although the mechanism of reactive aldehydes-induced cytoxicity has been extensively studied, little is known about how cells respond to toxic insults by $\alpha, \beta-$ unsaturated aldehydes. Cells have developed elaborate methods to resist cytotoxic aldehydes by the presence of cytoprotective genes encoding enzymes involved in aldehyde metabolism and detoxification. Multiple pathways are involved in the metabolism of acrolein and HNE including oxidation to carboxylic acid, reduction to an alcohol and the conjugation to GSH. The enzymes involved include glutathione $S$ transferase (GST), ALDH, aldo-keto reductases (AKR), alcohol dehydrogenase (ADH) and cytochromes P450 (CYP) (Amunom et al., 2007;Marchitti et al., 2008; Srivastava et al., 2001). The importance of these enzymes in acrolein and HNE detoxification is cell type- and species-dependent. Intravenous administration of acrolein into rats demonstrates that AKRs also play a major role in the metabolism of acrolein especially in the heart (Stevens and Maier, 2008). However, in vitro studies of HNE metabolism performed with isolated hepatocytes and perfused mice livers suggest that both Aldhs and GST are the main enzymes involved in lipid aldehyde metabolism in hepatic tissues (Hartley et al., 1995).

ALDHs gene superfamily encodes enzymes that catalyze the irreversible oxidation of endogenous and exogenous aldehydes to their corresponding non-toxic carboxylic acids using either $\mathrm{NAD}^{+}$or $\mathrm{NADP}^{+}$as cofactor. These enzymes play critical roles in the cellular protection against oxidative damage induced by cytotoxic aldehydes by metabolizing these aldehydes to a form that is easily excreted. The active ALDH enzyme is composed of subunits that form either a homodimer or homotetramer protein. Each subunit is characterized by a Rossmann fold and contains an NAD(P)-binding 
domain, a catalytic domain and an oligomerization domain (Marchitti et al., 2008). A funnel-shaped opening at the interface of these domains leads to a putative catalytic pocket and is essential for substrate specificity. The ALDH Rossmann fold contains the structural motif, (GxxxxG; $x$ represent any protein residue) which is necessary for cofactor binding. An invariant cysteine is found at the catalytic site of all ALDHs and is essential for the formation of the thiohemiacetal intermediate (Marchitti et al., 2008).

Members of the ALDH gene superfamily are composed of 555 genes in both eukaryotes and prokaryotes. To date, $172 \mathrm{ALDH}$ genes have been identified in eukaryotes (Sophos and Vasiliou, 2003) with 19 functional ALDH genes in the human genome (Alnouti and Klaassen, 2008;Ellis, 2007;Marchitti et al., 2008). Three isozymes, the cytosolic ALDH1A1 and ALDH3A1, and the mitochondrial ALDH2 are implicated in the detoxification of lipid-derived aldehydes. However, the role of Aldh isozymes in cellular protection against cytotoxic aldehydes especially in mice liver is still not completely understood.

ALDH2 is a homo- or heterotetramer highly expressed in the liver and extrahepatic tissues. The human ALDH2 is encoded in the nucleus and imported to the mitochondrial matrix with the help of a 17 amino acid N-terminal mitochondrial localization sequence. ALDH2 enzyme plays a major role in the alcohol metabolism pathway based on its low $\mathrm{K}_{\mathrm{m}}(<5 \mu \mathrm{M})$ for acetaldehyde oxidation (Klyosov et al., 1996). Asian individuals carrying a catalytically inactive form of the enzyme with a point mutation in ALDH2 (ALDH2*2) experience the facial flushing after alcohol ingestion, caused by elevated blood acetaldehyde (Goedde et al., 1992). Emerging evidence indicates that ALDH2 plays a crucial role in cytoprotection against myocardial ischemia- 
reperfusion injury probably by detoxifying reactive aldehydes such as HNE (Chen et al., 2008).

ALDH3A1 is a homodimer that oxidizes medium chain aliphatic and aromatic aldehydes, and utilizes either $\mathrm{NAD}^{+}$or $\mathrm{NADP}^{+}$as cofactor. Aldh3a1 is poorly expressed in normal murine liver but is constitively expressed in the cornea where it protects the eye from UV-induced oxidative damage by scavenging hydroxyl radicals and detoxification of lipid peroxidation-derived aldehydes, such as HNE (Alnouti and Klaassen, 2008;Lassen et al., 2007). ALDH3A1 efficiently metabolizes cytotoxic lipid aldehydes and protects cells against HNE-induced apoptosis (Townsend et al., 2001). ALDH3A1 plays a major role in the metabolism of the anticancer drug cyclophosphamide and increased expression of ALDH3A1 with ALDH1A1 is responsible for the resistant phenotype exhibited by most cells to cyclophosphamide toxicity (Marchitti et al., 2008).

The cytosolic ALDH1A1 enzyme is a homotetramer protein highly expressed in the liver, lung and retina. ALDH1A1 plays an important role in retinoic acid (RA) biosynthesis by efficiently catalyzing the oxidation of both all-trans- and 9-cis-retinal (Marchitti et al., 2008; Vasiliou et al., 2004). Thus, ALDH1A1 regulates normal growth, differentiation and development of adult epithelia by synthesizing RA, a ligand for the nuclear RA receptor (RAR) and retinoid X receptor (RXR). Aldh1a1 gene is expressed at higher levels in human hematopoietic stem cells (HSCs), and is critical for stem cell differentiation and function (Chute et al., 2006). The human ALDH1A1 utilizes either $\mathrm{NAD}^{+}$or $\mathrm{NADP}^{+}$as cofactor, but prefers $\mathrm{NAD}^{+}$over $\mathrm{NADP}^{+}$(Xiao et al., 2009). Previous studies revealed that the human ALDH1A1 provides only moderate protection 
against trans-2-nonenal and not against other lipid aldehydes, such as HNE, acrolein and trans-2-hexenal (Townsend et al., 2001). However, there is compelling evidence to suggest that ALDH1A1 plays a prominent role in the cellular defense against oxidative damage induced by cytotoxic aldehydes. Studies with Aldh $1 \mathrm{a} 1^{-/-}$mice indicate that Aldhla1 protects the eye lens and cornea from cataract formation due to ageing- or UV radiation-induced oxidative stress by detoxifying cytotoxic lipid aldehydes (Choudhary et al., 2005; Lassen et al., 2007). The human lens ALDH1A1 efficiently oxidizes lipidderived aldehydes, including HNE $\left(\mathrm{K}_{\mathrm{m}} 4.8 \mu \mathrm{M}\right)$, trans-2-heptenal $\left(\mathrm{K}_{\mathrm{m}} 177 \mu \mathrm{M}\right)$ and MDA $\left(\mathrm{K}_{\mathrm{m}} 3.5 \mu \mathrm{M}\right)$ (Marchitti et al., 2008;Xiao et al., 2009).

In addition to its role in detoxification of lipid aldehydes, Aldhlal plays an important role in drug and xenobiotic metabolism. Over-expression of Aldh1a1 causes resistance to the anticancer drug, cyclophosphamide (CP) by detoxifying its major active aldehyde metabolite, acrolein (Moreb et al., 2007). Aldh1a1 is one of the major enzymes involved in the metabolism of toxic ethanol metabolite, acetaldehyde $\left(\mathrm{K}_{\mathrm{m}} 50-180 \mu \mathrm{M}\right)$ (Browning and Horton, 2004;Marchitti et al., 2008). ALDH1A1 also possesses a $p$ nitrophenyl acetate esterase activity and is involved in the bioactivation of the antianginal drug, nitroglycerin (Beretta et al., 2008). Furthermore, recent studies indicate that ALDH1A1 is a major enzyme responsible for detoxification of 3-deoxyglucosone, which is produced by the process of glycoxidation, and 3-deoxyglucosone is implicated in the pathogenesis of diabetes (Collard et al., 2007). We hypothesized that Aldh1al is the major enzyme involved in acrolein and other lipid aldehyde detoxification in mouse liver. Thus, an understanding of the molecular mechanism regulating Aldh1a1 gene expression is of importance in potentially attenuating the toxic action of carbonyl compounds. 


\section{The Aldehyde dehydrogenase $1 \mathrm{a} 1$ gene regulation}

Even though Aldh1a1 is constitutively expressed in various tissues especially the liver, the expression of Aldhlal gene is known to be altered by a wide variety of endogenous and exogenous stimuli. The levels of Aldh1a1 mRNA were markedly lower in mice with elevated levels of hepatic RA, suggesting that elevated hepatic RA downregulates Aldh1a1 expression in a feedback pathway to control RA biosynthesis (Andreola et al., 1997). Aldh1a1 is a major enzyme involved in RA biosynthesis. Transient transfection studies revealed that RAR $\alpha$ and the CCAAT/enhancer-binding protein $\beta(\mathrm{C} / \mathrm{EBP} \beta)$ transactivate the Aldh1 gene. The activation of Aldh1al gene expression by RAR was further supported by the fact the $\mathrm{RXR} \alpha^{-/-}$mice display decreased liver Aldh1a1 levels (Gyamfi et al., 2006). The molecular mechanism of RA-induced down-regulation of Aldh1al was attributed to RA-mediated decrease in the mRNA expression of C/EBP $\beta$ (Elizondo et al., 2000;Elizondo et al., 2009).

Aldh1a1 was previously shown to be induced by phenobarbital (PB) treatment in rat (Pappas et al., 2001). Alnouti and Klaassen also reported induction of Aldh1a1 gene in mouse liver by constitutive androstane receptor (CAR) activators, presumably operating through a CAR binding site in the 5'-flanking region of Aldh1a1 gene (Alnouti and Klaassen, 2008). The 5'-flanking region of the mouse Aldh1a1 gene was previously characterized and is highly conserved to that of the rat and human ALDH1 (Hsu et al., 1999; Hsu et al., 2000). It contains putative cis-acting regulatory elements such as GATA, AP-1, Nrf2, SP-1 NF- $\mathrm{K}$ B, AhR-Arnt, CAR and Stat5 binding sites. There is also the presence of an Oct 1 and a CCAAT binding sites which are highly conserved in the rat 
and human gene, and are essential for basal promoter activity (Guimond et al., 2002; Hsu et al., 1999).

Aldhlal expression is reported to be enhanced by chemicals which are known to activate redox-sensitive transcription factors, such as Nuclear Factor-E2-related factor-2 (Nrf2). For example, oxidative and electrophilic stress were shown to modulate the expression of Aldh1al and other cytoprotective genes by activation of $\mathrm{Nrf} 2$ (Hu et al., 2006; Lamle et al., 2008;Leonard et al., 2006;Reisman et al., 2009; Thimmulappa et al., 2002). We also showed by microarray profiling in mice liver that Aldhlal gene expression was induced by electrophiles, such as BHA and acrolein (Amunom, Makia and Prough, unpublished report). However, the signaling pathway involved in electrophile-induced expression of Aldhlal gene is not completely understood. Electrophiles and oxidants modulate the expression of cytoprotective genes by activation of redox-sensitive transcription factors, such as Nrf2, AP-1 and NF-кB.

\section{The Nuclear Factor-E2-related factor-2 (Nrf2) transcription factor}

The transcription factor Nrf2 belongs to the cap-n-collar (CNC) family of basic leucine zipper (bZIP) proteins. In addition to oxidants, $\mathrm{Nrf2}$ is activated by chemical compounds that undergo redox cycling or be metabolized to reactive or electrophilic intermediate (Rushmore et al., 1990;Rushmore and Pickett, 1991). A wide variety of natural and synthetic compounds are known activators of $\mathrm{Nrf} 2$, such as BHA and its metabolite tert-butylhydroquinone (t-BHQ), sulforaphane (SFN), 1,2 dithiole-3-thione (D3T), phenethylisothiocyanate (PEITC) and reactive aldehydes. The mechanism of activation of Nrf2 by aldehydes and other electrophiles is not completely understood. 
However, the activation of $\mathrm{Nrf} 2$ by oxidative stress and electrophiles is proposed to occur by one of two mechanisms (Nguyen et al., 2003;Nguyen et al., 2004;Nguyen et al., 2009). In the absence of oxidative and electrophilic stress, $\mathrm{Nrf} 2$ is localized in the cytoplasm in a complex with Kelch-like ECH-associated protein 1 (Keap-1) that facilitates the degradation of $\mathrm{Nrf} 2$ via the proteosomal system. Thus, under basal conditions the levels of $\mathrm{Nrf} 2$ is kept relatively low. Oxidative and electrophilic stress causes modification of cysteine residues within the hinge region of Keap-1, disrupting its association with $\mathrm{Nrf} 2$ and leading to nuclear translocation of $\mathrm{Nrf} 2$.

Electrophiles, such as reactive aldehydes and BHA can also activate Nrf2 indirectly by activation of protein kinase signaling pathways (Huang et al., 2002;Huang et al., 2000). The depletion of GSH by reactive aldehydes and other electrophiles causes oxidative stress that activates stress-signaling pathways involving kinases. Electrophiles have been shown to directly activate Raf1 (a MAPK kinase kinase), which then phosphorylates and activates MEK (a MAPK kinase) (Yu et al., 1997). The involvement of mitogen-activated protein kinase (MAPK), such as p38, c-Jun N-terminal kinase (JNK) and extracellular signal-regulated kinases 1 and 2 (ERK1/2) in the activation of Nrf2 is well established (Wu et al., 2006;Yu et al., 1997;Yuan et al., 2006). Furthermore, acrolein-mediated activation of Nrf2 was dependent on the phosphatidylinositol-3-kinase (PI-3K) and protein kinase C (PKC) signal pathways (Zhang and Forman, 2008). The phosphorylation of Nrf2 at Ser 40 by PKC has been demonstrated to activate Nrf2 (Huang et al., 2002;Huang et al., 2000).

The activated Nrf2 heterodimerizes with small Maf (MafF, MafG and MafK) proteins and coordinates expression of antioxidant and electrophile detoxification genes 
by binding to the antioxidant/electrophile response element (ARE/EpRE) commonly found in the promoter or enhancer region of these genes (Rushmore et al., 1991). Gene expression profiling in the liver of $\mathrm{Nrf}^{+/+}$and $\mathrm{Nrf}^{-/-}$mice exposed to prooxidants revealed that $\mathrm{Nrf} 2$ regulates hundreds of genes, including several antioxidant and electrophile detoxification enzymes (Hu et al., 2006). The most notable of these genes are the glutathione $S$-transferases (GSTA2, GSTP, Gsta1 and Gstm1), $\gamma$-glutamyl synthetase $(\gamma$-GCS), NADPH: quinone oxidoreductases (NQO-1), heme oxygenase (HO-1), glutathione peroxidases (GPx) and glutathione reductases (GR). We and others have shown that the Nrf2 activators, BHA and acrolein, increased mRNA expression of Aldhlal. The signaling pathways involved in the transcriptional regulation of Aldhlal gene by BHA and acrolein have not been previously studied. Moreover, it is not yet known whether Aldhlal is a direct target of Nrf2. However, aside from Nrf2, BHA and reactive aldehydes are also known to induce the transcriptional activity of the AP-1 and NF-kB transcription factors (Choi and Moore, 1993; Iles and Liu, 2005).

\section{The Activator Protein 1 (AP-1) transcription factor}

The AP-1 transcription factors are basic leucine zipper (bZIP) proteins that include members of the fos (c-fos, Fos-B, Fra-1 and Fra-2), jun (c-Jun, Jun B and Jun D) and the activating transcription factors (ATF1-5 and B-ATF). Members of the $j$ un family are known to regulate gene expression as either homodimers or as heterodimers with members of the fos family. The dimeric AP-1 binds to cis-acting 12-Otetradecanoylphorbol 13-acetate (TPA)-responsive element (TRE) in the promoter of target genes (Eferl and Wagner, 2003) (Figure 1) (Eferl and Wagner, 2003). The TRE 
was originally described in the human collagenase gene promoter and has the sequence, 5'-ATGAC/GTCA-3'. C-Jun is also known to form heterodimer protein complexes with Nrf2 and ATF2. The heterodimers of c-Jun with ATF-2 and Nrf2 are known to bind to the cyclic AMP response element (CRE) and ARE, respectively (Figure 1). The c-Jun protein has a b-ZIP DNA-binding domain and a transactivation domain containing phosphorylation sites for stress-activated protein kinases (SAPKs) such as JNK, p38 and ERK1/2. In response to oxidative and electrophilic stress, the SAPKs phosphorylate cJun and enhance its transactivating capacity. In addition, reactive aldehydes can enhance the transcriptional activity of AP-1 by induction of AP-1gene expression (Pugazhenthi et al., 2006;Ranganna et al., 2002;Tirumalai et al., 2002; Wu et al., 2006;Zhang and Forman, 2008).

AP-1 proteins have been implicated in several signal transduction pathways associated with cellular growth, differentiation and cellular stress (Eferl and Wagner, 2003). AP-1 can either promote or antagonize cell survival in a tissue- and stressdependent manner. The mechanism that accounts for the dual role of AP-1 in apoptosis and survival signaling has not been established. Previous studies showed that c-Jun acts downstream of JNK to induce apoptosis in TNF $\alpha$-stimulated thymocytes (Hasselblatt et al., 2007). However, $c$-jun null mice die at midgestation and display increased apoptosis of fetal liver cells (Eferl and Wagner, 2003). A more recent study indicates that oxidative stress-mediated induction of c-Jun/AP-1 promotes hepatocyte survival during acute hepatitis by transcriptional regulation of inducible nitric oxide synthase (Nos 2) gene (Hasselblatt et al., 2007). These results indicate that c-Jun has hepatoprotective functions 

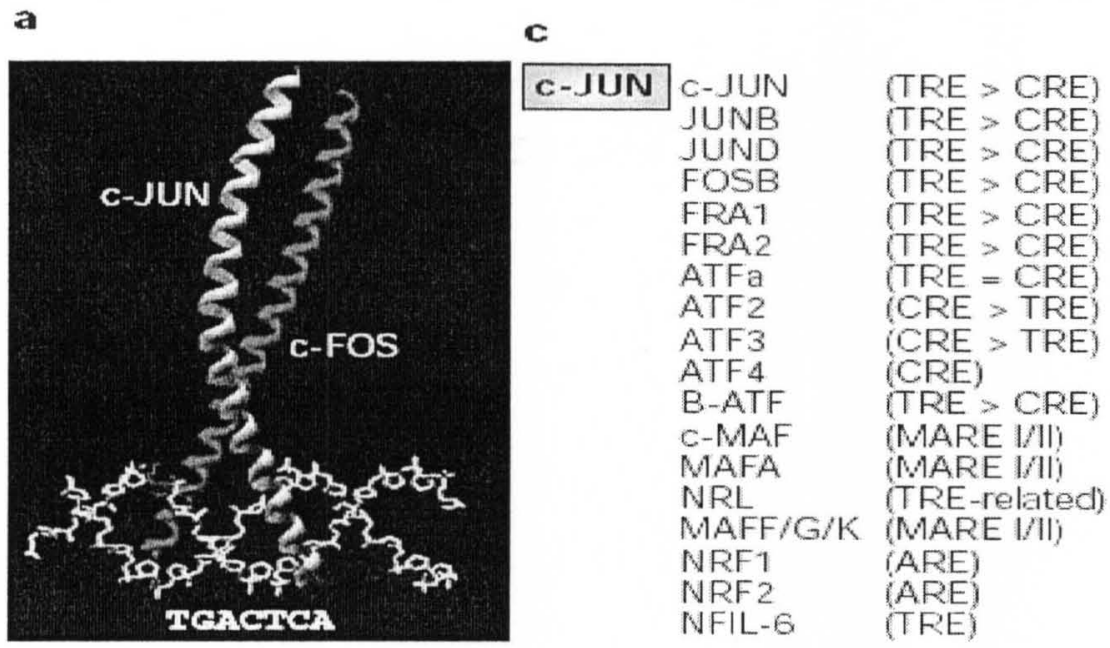

\begin{tabular}{|c|c|c|c|c|}
\hline b & & $d$ & & \\
\hline TRE: & TGACTCA & c-FOS & $c-J \cup N$ & $(\mathrm{TRE}>\mathrm{CRE})$ \\
\hline & ACTGAGT & & $\begin{array}{l}\text { JUNB } \\
\text { JUND }\end{array}$ & $\begin{array}{l}\text { (TRE }>\text { CRE }) \\
(T R E>C R E)\end{array}$ \\
\hline SRE: & $\overrightarrow{T G A C G T C A}$ & & ATFa & (No bincling) \\
\hline & ACTGCAGT & & $\begin{array}{l}\text { ATF2 } \\
\text { ATF4 }\end{array}$ & $\underset{(\mathrm{CRE})}{(\mathrm{CRE})}>\mathrm{TRE})$ \\
\hline MARE I: & 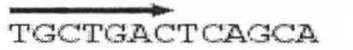 & & C-MAF & (MARE VII) \\
\hline & ACGACTGAGTCGT & & $\begin{array}{l}\text { MAFB } \\
\text { NRL }\end{array}$ & $\begin{array}{l}\text { (MARE VII) } \\
\text { (TRE-reliated) }\end{array}$ \\
\hline MARE II: & TGCTGACGTCAGCA & & MAFF/G/K & (MARE $/ / \| j$ \\
\hline & ACGACTGCAGTCGT & & $\begin{array}{l}\text { NRF2 } \\
\text { NFIL6 }\end{array}$ & $\begin{array}{l}\text { (ARE) } \\
\text { (TRE) }\end{array}$ \\
\hline ARE: & $\begin{array}{l}a / \text { GTGACnnnGC } \\
\text { t/CACTGnnnCG }\end{array}$ & & & \\
\hline
\end{tabular}

Figure 1. The basic leucine zipper (bZIP) family proteins and their binding partners. The jun family members can form either homodimer or heterodimers with members of the fos family. The dimeric AP-1 binds to cis-acting 12-O-tetradecanoylphorbol 13-acetate (TPA)-responsive elements (TRE) in the promoter of target genes (Eferl and Wagner, 2003). c-Jun also form heterodimers with ATF-2 and Nrf2 which binds to the cyclic AMP response element (CRE) and antioxidant response element (ARE), respectively. 
especially against oxidative stress (Eferl and Wagner, 2003;Hasselblatt et al., 2007;Marden et al., 2008; Tsuji, 2005). We propose that increased transcriptional activation of the aldehyde-metabolizing gene, Aldhlal, by reactive aldehydes is mediated by activation of c-Jun/AP-1 proteins.

\section{The nuclear factor kappa $B(\mathrm{NF}-\mathrm{kB})$}

Nuclear Factor kappa B (NF-kB) proteins are redox-sensitive transcription factors, which play key roles in immune and inflammatory responses. There are five NF$\kappa \mathrm{B}$ proteins in mammals: RelA (p65), RelB (p68), c-Rel (p75), p50 and p52 (Karin, 1999). They modulate gene expression by forming a variety of heterodimers and homodimers with each other. In most tissues, the predominant NF-kB dimer is a $\mathrm{p} 50 / \mathrm{p} 65$ heterodimer. NF-KB proteins are also known to modulate gene expression by forming active heterodimers with AP-1 proteins (Rahmani et al., 2001). NF- $\mathrm{kB}$ proteins are held in an inactive state in the cytoplasm by inhibitory proteins known as IкB. Following exposure to oxidative stimuli and electrophiles, IкB undergoes phosphorylation, ubiquitination and subsequent degradation of IאB (Karin, 1999). The degradation of IאB leads to the release of the $\mathrm{p} 50 / \mathrm{p} 65$ complexes, resulting in nuclear translocation and transcriptional activation of specific target genes. These target genes are involved in inflammatory responses to infection and stess.

We hypothesize that mouse liver displays a protective response to toxic aldehydes by enhanced metabolic inactivation of these toxic species, mediated by induction of Aldh1a1 enzymes. Moreover, electrophile-induced expression of Aldh1al gene is mediated by c-Jun/AP-1.The goal of these studies is two-fold. 1. To examine whether 
lipid aldehydes and their GSH conjugates are substrates for Aldh1a1. 2. To decipher the signaling pathways involved in electrophile-mediated induction of Aldhlal gene expression in mouse liver. 


\section{CHAPTER II}

\section{MURINE HEPATIC ALDEHYDE DEHYDROGENASE 1A1 (Aldh1a1) IS A MAJOR CONTRIBUTOR TO OXIDATION OF ALDEHYDES FORMED BY LIPID PEROXIDATION}

\section{INTRODUCTION}

The liver, as the major site for metabolism and biotransformation of drugs and foreign compounds, is constantly exposed to reactive oxygen species (ROS), resulting in oxidative stress. During oxidative stress, these ROS, which include hydrogen peroxide $\left(\mathrm{H}_{2} \mathrm{O}_{2}\right)$, superoxide radicals $\left(\mathrm{O}_{2}\right)$ and hydroxyl radicals $(\mathrm{OH}$.$) , can covalently modify$ proteins, lipids and DNA. The peroxidation of polyunsaturated fatty acids in membrane lipid by ROS produces unstable lipid hydroperoxides that decompose into aldehydes such as malondialdehyde (MDA), hexanal, trans-2-hexenal, propen-2 al (acrolein) and 4hydroxy-2-nonenal (HNE) (Esterbauer et al., 1991;Esterbauer, 1993). Among these aldehydes, HNE and acrolein are highly electrophilic $\alpha, \beta$-unsaturated aldehyde that can undergo Schiff base and Michael addition reactions with nucleophilic groups on proteins and DNA (Esterbauer, 1993). There is increasing evidence that the pathophysiological effects of ROS in cells is mediated by these cytotoxic aldehydes because they are more chemically stable than ROS and thus, can diffuse to distant sites from where they are formed (Ellis, 2007; Uchida et al., 1994). 
In addition, humans are exposed to these aldehydes as environmental pollutants and by endogenous processes generating reactive aldehydes in the liver. For example, chronic alcohol consumption, high fat diet or exposure to foreign compounds such as carbon tetrachloride $\left(\mathrm{CCl}_{4}\right)$, allyl alcohol and the widely used anticancer drug, cyclophosphamide, markedly elevate the intracellular concentrations of cytotoxic aldehydes (Dwivedi et al., 2006;Nanji et al., 1994;Sampey et al., 2007;Stevens and Maier, 2008). Reactive $\alpha, \beta$-unsaturated aldehydes, such as acrolein and crotonaldehyde are also present in cigarette smoke, vehicle exhaust emission, overheated foods and oil, drinking water and in effluents from industrial plants (Feron et al., 1991;Stevens and Maier, 2008). The maximum daily human consumption of unsaturated aldehydes is estimated at $\approx 5 \mathrm{mg} / \mathrm{kg}$ whereas the total aldehyde (saturated and unsaturated) consumption is estimated at $\approx 7 \mathrm{mg} / \mathrm{kg}$ (Wang et al., 2008;Conklin et al., 2010).

The levels of reactive lipid aldehydes are elevated in various oxidative stressmediated diseases, including steatohepatitis (Chen et al., 1997), atherosclerosis (Uchida et al., 1994), Alzheimer's disease (Sayre et al., 1997), cataractogenesis (Srivastata et al., 1996), diabetes (Toyokuni et al., 2000) and cancer (Hammer et al., 1997). In fact, accumulation of reactive aldehydes is associated with the pathogenesis of these diseases. The toxicity of $\alpha, \beta$-unsaturated aldehyde lies in their ability to form Michael adducts with thiol and amino groups of proteins resulting in alteration of several cellular processes. For example, enzymes such as glyceraldehyde-3-phosphate, glucose-6-phosphate dehydrogenases and cytochrome $\mathrm{c}$ oxidase containing lysine and cysteine residues in their active sites are readily inactivated by conjugation with these reactive aldehydes (Chen et al., 2001; Szweda et al., 1993;Uchida and Stadtman, 1993). In addition, $\alpha, \beta-$ 
unsaturated aldehydes can induce oxidative stress in cells by depleting cellular reduced glutathione, thereby altering signal transduction pathways in cells. At low concentrations, acrolein is known to trigger apoptotic cell death by mechanisms that involve activation of mitochondrial death pathways and caspases (Burcham and Fontaine, 2001;Stevens and Maier, 2008;Tanel and Averill-Bates, 2007). Caspases, particularly caspase 3, which can cleave substrates, such as poly(ADP-ribose) polymerase (PARP), actin and laminin, are widely used as markers for apoptosis in different cell types. However, at high concentrations acrolein causes necrotic cell death. This phenomenon has also been observed with HNE (Cheng et al., 2001; Thornberry and Lazebnik, 1998; Zhang et al., 2010).

Despite their toxicity, many cytotoxic lipid-derived aldehydes can be successfully metabolized to less toxic compounds by the action of oxidative, reductive and conjugative enzymes. These enzymes include glutathione $S$-transferases (GST), aldehyde dehydrogenases (ALDH), aldo-keto reductases (AKR), alcohol dehydrogenases (ADH) and cytochrome P450 (CYP) (Amunom et al., 2007; Conklin et al., 2007; Marchitti et al., 2008; Srivastava et al., 1998; Srivastava et al., 2001). The relative importance of these enzymes in reactive aldehyde metabolism is cell type- and species-dependent. It is now known that the conjugation of acrolein with glutathione may not be a true detoxification process because acrolein-glutathione conjugates can undergo renal processing to form reactive species (Ramu et al., 1995). In addition, the conjugation process is compromised when GSH concentrations are depleted during oxidative stress. ALDH oxidizes a range of toxic aldehydes to their corresponding non-toxic carboxylic acids using either $\mathrm{NAD}^{+}$ or $\mathrm{NADP}^{+}$as cofactors. ALDHs play a critical role in the cellular protection against these 
toxic species. There are 19 ALDH genes in the human genome (Marchitti et al., 2008). To date, three isozymes, the cytosolic ALDH1A1 and ALDH3A1, and the mitochondrial ALDH2 are suggested to be the main lipid aldehyde-oxidizing enzymes expressed in the mouse liver.

The role of these enzymes in the cellular defense against oxidative damage induced by cytotoxic aldehydes is controversial. Previous studies by Townsend et al revealed that ALDH1 A1 over-expression provides only moderate protection against trans-2-nonenal and not against other lipid aldehydes (Townsend et al., 2001). However, ALDH3A1 could protect RAW 264.7 murine macrophage cell line against HNE-induced apoptosis, consistent with the prevention of HNE-protein adduct formation (Townsend et al., 2001). Mouse Aldh3al is poorly expressed in normal liver and highly expressed in cancerous cells (Alnouti and Klaassen, 2008). It is also abundantly expressed in the cornea and protects the cornea against cytotoxic lipid peroxidation-derived aldehydes (Lassen et al., 2007). However, recent experiments with Aldh $1 \mathrm{a} 1^{/ /}$mice indicate that Aldh1al also protects the eye from cataract formation induced by oxidative stress by detoxifying cytotoxic lipid aldehydes (Choudhary et al., 2003; Choudhary et al., 2005). Moreover, the human lens ALDH1A1 efficiently oxidizes lipid-derived aldehydes, including HNE $\left(\mathrm{K}_{\mathrm{m}} 4.8 \mu \mathrm{M}\right)$, trans-2-heptenal $\left(\mathrm{K}_{\mathrm{m}} 177 \mu \mathrm{M}\right)$ and MDA $\left(\mathrm{K}_{\mathrm{m}} 3.5 \mu \mathrm{M}\right)$ (Xiao et al., 2009). In addition, over-expression of ALDH1 in neuroblastoma cells reduces both production of protein-HNE adducts and activation of caspase-3 (Zhang et al., 2010). Aldh1al is known to decrease the effectiveness of the anticancer drugs cyclophosphamide by detoxifying its major active metabolites acrolein (Moreb et al., 2007). These results indicate that Aldh1a1 has the potential to protect against aldehydes 
produced as a result of lipid peroxidation. However, it is unknown whether Aldhlal can protect against acrolein-induced toxicity in mouse liver. We hypothesized that Aldh1a1 is the major enzyme involved in acrolein and other lipid-derived aldehyde metabolism and detoxification in mouse liver.

Although considerable characterization of the rat ALDHs in cytosol and mitochondria has been published, the relative contribution of different Aldh isozymes in metabolism and detoxification of reactive aldehydes in mouse liver is unknown.

Moreover, the kinetic properties of murine Aldh orthologs in oxidation of reactive lipid aldehydes especially acrolein, have not been biochemically measured. Mouse is a more common laboratory model for research in medicine because of the availability of transgenic and until recently, the only source for gene-targeted and knockout mice. In the present study, we examined the role of murine hepatic Aldh isozymes in metabolism and detoxification of lipid-derived aldehydes, including acrolein and HNE, by enzyme kinetic and gene expression studies. In this chapter, we show by substrate preferences, gene expression patterns and in vitro knockdown experiments in Hepa-1c1c7 that murine Aldh1a1 is the major cytosolic Aldh in mouse liver involved in cellular defense against reactive aldehydes-induced toxicity.

\section{MATERIALS AND METHODS}

\section{Chemicals and Reagents}

Propionaldehyde, benzaldehyde, trans-2-hexenal, acetaldehyde, dithiothreitol (DTT), 3-(4,5-dimethylthiazol-2-yl)-2, 5-diphenyltetrazolium bromide (MTT), isopropyl $\beta$-D-1-thiogalactopyranoside (IPTG), N-ethylmaleimide (NEM), propen-2-al (acrolein) 
and malonaldehyde bis-(dimethyl acetal, MDA) were purchased from Sigma-Aldrich Company, Inc. (St Louis, MO). 4-hydroxy-2-nonenal (HNE) was obtained from Cayman Chemical Co (Ann Arbor, MI). Oxidized $\beta-\mathrm{NAD}^{+}$and $\beta-\mathrm{NADP}^{+}$were purchased from Codexis (Redwood City, CA). Anti-glyceraldehyde-3-phosphate dehydrogenase (GAPDH) antibody (clone 6C5, MAB374) was purchased from Millipore (Temecula, CA). Rabbit polyclonal antibody against caspase 3 (H-277; sc-7148) was purchased from Santa Cruz Biotechnology, Inc (Santa Cruz, CA). Cleaved caspase 3 (Asp175) antibodies (\#9661), and cytochrome $c$ treated and untreated Jurkat cell extracts (\#9663) were from Cell Signaling Technology (Danvers, MA). ALDH1 antibody against purified rat ALDH1 was produced in rabbits (Tweedie et al., 1991). Rabbit polyclonal antibody against acrolein-protein adducts was provided by Aruni Bhatnagar, Department of Medicine/Cardiovascular Medicine, University of Louisville. Stealth ${ }^{\mathrm{TM}}$ RNAi was synthesized by Invitrogen (Carlsbad, CA).

\section{Cloning, Expression and Purification of Recombinant Aldh1a1, Aldh2 and Aldh3a1}

The pCMV6-Aldh1al plasmid (MC202273) and pCMV6-Aldh2 (MR208315) containing the full length of mouse Aldh1al cDNA (NM_013467) and Myc-DDK-tagged mouse Aldh2 (NM_009656.2) Open Reading Frame (ORF), respectively, were purchased from OriGene (Rockville, MD). The ORF of Aldh1a1 was amplified by PCR using the following primers (forward: 5'-CATATGTCTTCGCCTGCACAACCTGCA-3'; reverse: 5'-GGTACCGGAGTTCTTCTGAGATATCTTCA-3'). On the other hand, the ORF for Aldh2 was obtained using the following primers (forward: 5'CATATGTCCGCCGCCGCCACCAGCGC-3'; reverse: 5'- 
GGTACCCGAGTTCTTCTGTGGCACTTTGA-3'). The forward primer for amplification of Aldh2 was designed to remove the mitochondrial signal sequence by PCR. Both forward primers contain an Nde1 site for cloning into the start codon (ATG) of pET30b vector (Novagen, Inc., Madison, WI) to ensure correct initiation of translation in $E$ coli. The reverse primer was designed to contain a Kpn1 site with deletion of the stop codon (TAA) to express a full length Aldh1a1 with a C-terminal his-tag. The $1.5 \mathrm{~kb}$ PCR product was then cloned into $\mathrm{pCR} 2.1$ vector using a TA cloning vector kit (Invitrogen, Carlsbad, CA). The sequence of the mouse Aldh1a1 and Aldh2 ORF was confirmed by sequence analysis at the University of Louisville, Center for Genetics and Molecular Medicine Nucleic Acid Core Facility. The PCR product was then digested with Nde1 and Kpnl, and subcloned into the pET30b expression plasmid (Novagen, Inc., Madison, WI). To generate the full length of mouse Aldh3al cDNA (NM_007436), total RNA was isolated from mouse primary hepatocytes treated with $50 \mu \mathrm{M} 1,2-$ benzanthracene (BA) for $24 \mathrm{~h}$. Total RNA was reverse transcribed to cDNA with OligodT primers using Advantage RT-for-PCR kit (Clontech, BD Bioscience, Palo Alto, CA). The ORF of Aldh3al was generated by PCR using as forward primer, 5'GGTACCAGCAATATCAGTAGCATCG-3' and as reverse primer, 5'CTCGAGTGAAGTAGCCCTCTCAATGC-3'.

The sequence of the mouse Aldh3a1 ORF PCR product was confirmed by sequence analysis. The $1.5 \mathrm{~kb}$ PCR product was ligated into pCR2.1 vector and subsequently digested with Kpn1 and Xhol, and subcloned into pET30a (Novagen) expression vector allowing synthesis of a full-length Aldh3a1 with an $\mathrm{N}$-terminal his-tag. One Shot ${ }^{\circ}$ BL21(DE3)pLysS competent E. coli (Invitrogen, Carlsbad, CA) were 
subsequently transformed with pET30b-Aldh1a1 or pET30a-Aldh3a1 plasmid and were grown until the $\mathrm{OD}_{600} \approx 0.6$. The recombinant protein expression was induced by addition of $0.5 \mathrm{mM}$ IPTG overnight. Cells were lysed with Bugbuster ${ }^{\circ}$ Protein extraction reagent (Novagen, Inc., Madison, WI) containing 2 mM DTT and 10\% glycerol, and purified using nickel-nitrilotriacetic acid (Ni-NTA) column (Qiagen, Valencia, CA). Proteins were eluted with $400 \mathrm{mM}$ imidazole and concentration was determined by Pierce ${ }^{8}$ BCA Protein Assay (Pierce, Rockford, IL). The purity of the recombinant protein was determined by SDS-PAGE electrophoresis stained with coomassie blue (Figure 2). We noted a single protein band at $\approx 55$ and $50 \mathrm{KDa}$ in the eluate fractions of the pET30b-Aldh1a1 and pET30-Aldh3a1 transformed E. coli, respectively (Figure 2). To further confirm the identity of Aldh1a1, extracts from BL21(DE3)pLysS cells transformed with pET30b-Aldh1a1, and purified rat ALDH1 (Tweedie et al., 1991) were resolved on SDS-PAGE gel, transferred onto nitrocellulose membrane and probed with antibody against purified rat ALDH1A1.

\section{Aldehyde Dehydrogenase Enzymatic Assay}

The enzymatic activities of Aldhs were measured spectrophotometrically using recombinant his-tag proteins by monitoring the reduction of $\mathrm{NAD}^{+}$or $\mathrm{NADP}^{+}$at $340 \mathrm{~nm}$ as previously described by Lindahl and Petersen (Lindahl and Petersen, 1991) using the molar absorptivity value of $6,220 \mathrm{M}^{-1} \mathrm{~cm}^{-1}$. Enzyme activity was assayed at $25^{\circ} \mathrm{C}$ in 50 mM sodium phosphate buffer ( $\mathrm{pH} 7.4$ ), containing $1 \mathrm{mM}$ EDTA and $0.01 \%$ BME with either $1 \mathrm{mM} \mathrm{NAD}^{+}$or $\mathrm{NADP}^{+}$as cofactor. The reaction was initiated by addition of varying concentrations of aldehyde substrate into a $1 \mathrm{ml}$ cuvette. The kinetic constants 


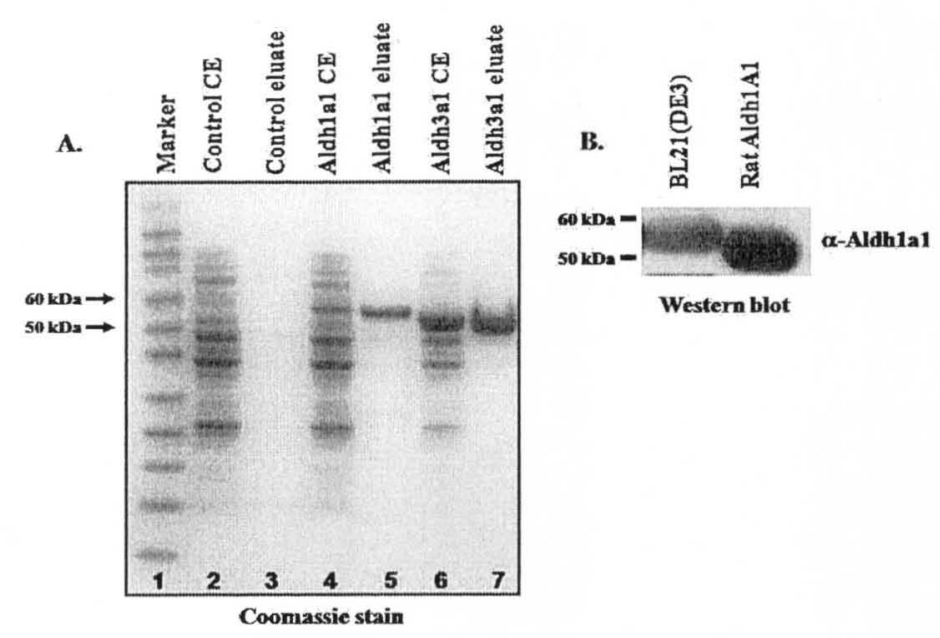

Figure 2. SDS-PAGE analyses of purified recombinant mouse Aldh. (A) Cell extracts (CE) from pET30a, pET30b-Aldh1a1 or pET30a-Aldh3a1 transformed BL21(DE3)pLysS E coli and eluate fractions purified using Ni-NTA resin were resolved on 4-12\% SDSPAGE and stained with coomassie blue. Lane 1: Prestained Marker; Lane 2: CE from pET30a transformed $E$ coli; Lane 3: eluate fractions from pET30a vector transformed $E$ coli; Lane 4: Lysates from pET30b-Aldh1a1 transformed E coli; Lane 5: eluate fractions from pET30b-Aldh1a1 transformed E coli; Lane 6: CE from pET30a-Aldh3a1 transformed $E$ coli; Lane 7: eluate fractions from pET30b-Aldh1a1 transformed $E$ coli. (B) Western blot analysis of recombinant Aldh1a1. Extracts from BL21(DE3)pLysS transformed with pET30b-Aldh1a1 and purified rat liver ALDH1A1 immunoblotted with antibody against rat ALDH1. Due to the presence of his-tag the migration of the recombinant mouse Aldh1a1 is slower than that of purified rat Aldh1. Lane 1: Eluate fractions from BL21(DE3)pLysS transformed with pET30b-Aldh1a1 plasmid; Lane 2: ALDH1A1 purified from rat liver. 
$\left(\mathrm{K}_{\mathrm{m}}, \mathrm{V}_{\max }\right.$ and $\mathrm{V}_{\max } / \mathrm{K}_{\mathrm{m}}$ ) of recombinant mouse Aldh1a1 and Aldh3a1 for oxidative metabolism of various aldehydes were examined. All reactions were performed in triplicate. Malondialdehyde (MDA) was prepared by acid hydrolysis of malonaldehyde bis-(dimethyl acetal) (Sigma-Aldrich Co, St. Louis, MO) by adding $17 \mu \mathrm{L}$ to $900 \mu \mathrm{L}$ of $0.1 \mathrm{~N} \mathrm{HCl}$ and incubating the solution at room temperature for $2 \mathrm{~h}$ (vortexing every 30 $\min$ ). The $\mathrm{pH}$ was neutralized with $1 \mathrm{~N} \mathrm{NaOH}$ prior to use to achieve a $100 \mathrm{mM}$ concentration. The cofactor preference of recombinant mouse ALDH was measured using varying concentrations of either $\mathrm{NAD}^{+}$or $\mathrm{NADP}^{+}$. The $\mathrm{K}_{\mathrm{m}}$ of Aldh1al for $\mathrm{NAD}^{+}$ and $\mathrm{NADP}^{+}$was defined using $1 \mathrm{mM}$ propionaldehyde while the cofactor preference of Aldh3al was assessed by measuring its $\mathrm{K}_{\mathrm{m}}$ for either $\mathrm{NAD}^{+}$or $\mathrm{NADP}^{+}$using $2.5 \mathrm{mM}$ benzaldehyde as substrate.

\section{Stealth $^{\mathrm{TM}}$ siRNA knockdown of mouse Aldh1a1}

Stealth ${ }^{\mathrm{TM}}$ RNA oligonucleotides are 25 bp double-stranded RNA oligonucleotides and were designed using BLOCK-i $\mathrm{T}^{\mathrm{TM}}$ RNAi Designer (Invitrogen, Carlsbad, CA). The Stealth siRNA designed to target mouse Aldhla1 (NM_013467) was synthesized by Invitrogen (sense strand, 5'-UAAAGAUGCCAGGUGAAGAGCCGUG-3'; antisense strand, 5'-CACGGCUCUUCACCUGGCAUCUUUA-3'). The sequences of the Stealth siRNA control are: sense strand, 5'-CACUCUCCUCAUCGGACCUUGGUUA-3'; antisense strand, 5'-UAACCAAGGUCCGAUGAGGAGAGUG-3'). Before transfection, mouse hepatoma (Hepa-1c1c7) cells (American Type Culture Collection, Rockville, MD) were maintained in Dulbellco Modified Eagle's Media (DMEM) supplemented with 10\%

fetal bovine serum and 1\% Antibiotic-Antimycotic (Invitrogen; Carlsbad CA). Cells were 
transfected with either 150 pmol $(50 \mathrm{nM})$ Stealth $^{\mathrm{TM}}$ siRNA control (siControl) or Stealth ${ }^{\mathrm{TM}}$ siRNA specific to Aldh1al (siAldh1a1) using Lipofectamine ${ }^{\mathrm{TM}}$ RNAiMAX (Invitrogen) according to manufacturer's instructions. To test the effect of stealth siRNA on Aldh1a1 gene expression, the mRNA levels and protein levels were analyzed $48 \mathrm{~h}$ after transfections by qRT-PCR and Western blot, respectively.

\section{Measurement of Cell Viability by MTT assay}

Hepa-1c1c7 cell viability was evaluated using an MTT assay according to manufacturer's instruction. Hepa-lc1c7 cells $(5,000$ cells/ $100 \mu \mathrm{l})$ were plated in a $96-$ well plate. Cells were treated with increasing concentrations of acrolein $(0$ to $100 \mu \mathrm{M})$ in serum free media for $24 \mathrm{~h}$. Cells were then incubated with MTT $(0.2 \mathrm{mg} / \mathrm{ml})$ for $2 \mathrm{~h}$ and cell viability was assessed by measuring the absorbance at $570 \mathrm{~nm}$.

\section{RNA Isolation and Quantitative Real Time PCR (QRT-PCR).}

RNA was isolated from Hepa-1c1c7 cells or mouse liver using TRI reagent (Molecular Research Center, Inc., Cincinnati, OH). The mRNA levels of Aldh1a1, Aldh1b1, Aldh2 and Aldh3al were assessed by qRT-PCR. Total RNA isolated from cells was reverse transcribed to cDNA with random hexamer primers using the Advantage RTfor-PCR kit (Clontech, BD Bioscience, Palo Alto, CA). RNase $\mathrm{H}$ was then used to degrade any residual RNA in the CDNA mix. QRT-PCR was performed using the ABI 7900HT Sequence Detector System (Applied Biosystems, Foster City, CA) with gene specific FAM-labeled LUX primers synthesized by Invitrogen (Carlsbad, CA). A plot of the CT versus quantity of RNA was generated to verify linearity of amplification. All 
qRT-PCR experiments were performed in triplicate using cDNA sample from independent RNA set and analyzed by the absolute quantitation standard curve method. The gene expression levels were normalized to $18 \mathrm{~S}$ rRNA as endogenous control and data were expressed as the mean \pm SD. Samples were analyzed by Student's $t$ test and values of $\mathrm{p}<0.05$ were considered to be statistically significant.

\section{Western Immunoblotting.}

Whole cell extracts were prepared from Hepa-1c1c7 cells using 1X RIPA buffer (20 mM Tris- $\mathrm{HCl}$, pH 7.5; 150 mM NaCl, 1 mM Na 2 EDTA, 1 mM EGTA, 1\% NP-40, $1 \%$ sodium deoxycholate, $2.5 \mathrm{mM}$ sodium pyrophosphate, $1 \mathrm{mM} \beta$-glycerophosphate, 1 $\mathrm{mM} \mathrm{Na}{ }_{3} \mathrm{VO} 4,1 \mu \mathrm{g} / \mathrm{ml}$ leupeptin; Cell Signaling Technology, Beverly, MA) containing protease inhibitors (Protease Inhibitor Cocktail Set III, EDTA free; Calbiochem, La Jolla, CA). For determination of acrolein-protein adducts in cells, a modified 1X RIPA Lysis buffer containing $50 \mathrm{mM}$ NEM was used. Protein concentration was measured using Pierce ${ }^{\circledR}$ BCA Protein Assay (Pierce, Rockford, IL). Lysates were resolved on 4-12\% NuPAGE ${ }^{\circledR}$ Novex ${ }^{\circledR}$ Bis-Tris mini gels and transferred onto Amersham Hybond ${ }^{\mathrm{TM}}$-ECL nitrocellulose membranes (GE Amersham Pharmacia Biotech, Piscataway, NJ). Membranes were probed with antibodies against rat ALDH1 (1:2000), Caspase 3 $(1: 1000)$ or GAPDH $(1: 10000)$ at room temperature for $2 \mathrm{~h}$ or acrolein-protein adducts (1:2000) and cleaved Caspase $3(1: 1000)$ at $4^{\circ} \mathrm{C}$ overnight. The membrane was incubated with horseradish peroxidase-conjugated goat anti-rabbit (Sc-2004, Santa Cruz Biotechnology) or goat anti-mouse (Sc-2005, Santa Cruz Biotechnology, Santa Cruz, 
CA) secondary antibody and the transferred proteins were visualized with Amersham ECL Plus western blotting detection system.

\section{RESULTS}

\section{Expression, Purification and Biochemical Characterization of Recombinant}

\section{Aldh1a1, Aldh2 and Aldh3a1}

We examined the kinetic parameters $\left(\mathrm{K}_{\mathrm{m}}, \mathrm{V}_{\max }\right.$ and $\left.\mathrm{V}_{\max } / \mathrm{K}_{\mathrm{m}}\right)$ of recombinant mouse Aldh1a1, Aldh2 and Aldh3a1 (Table 1) for oxidative metabolism of a wide range of aldehyde substrates and the GS conjugates of $\mathrm{HNE}$ using either $\mathrm{NAD}^{+}$or $\mathrm{NADP}^{+}$as pyridine nucleotide cofactor. The cofactor preference of recombinant mouse ALDH was also assessed using varying concentrations of either $\mathrm{NAD}^{+}$or $\mathrm{NADP}^{+}$. Kinetic parameters indicate that mouse Aldh1a1 exhibited high affinity for short chain aldehydes, such as propionaldehyde $\left(\mathrm{K}_{\mathrm{m}}=141 \mu \mathrm{M}\right)$ and acetaldehyde $\left(\mathrm{K}_{\mathrm{m}}=202 \mu \mathrm{M}\right)$ and the lipid aldehydes; acrolein $\left(\mathrm{K}_{\mathrm{m}}=23.2 \mu \mathrm{M}\right)$, trans-2-hexenal $\left(\mathrm{K}_{\mathrm{m}}=31.2 \mu \mathrm{M}\right), \mathrm{HNE}\left(\mathrm{K}_{\mathrm{m}}=2.4\right.$ $\mu \mathrm{M})$ and MDA $\left(\mathrm{K}_{\mathrm{m}}=7.5 \mu \mathrm{M}\right)$ as shown in Table 1. The catalytic efficiency for Aldh1a1 was highest for HNE $\left(\mathrm{V}_{\max } / \mathrm{K}_{\mathrm{m}}=218\right)$ and lowest for benzaldehyde $\left(\mathrm{V}_{\max } / \mathrm{K}_{\mathrm{m}}=0.3\right)$. Mouse Aldhlal had an apparent $\mathrm{K}_{\mathrm{m}}$ of $752 \mu \mathrm{M}$ for benzaldehyde, indicating that the aromatic aldehydes, benzaldehyde is a poor substrate for Aldh1a1. Aldh1a1 preferred $\mathrm{NAD}^{+}\left(\mathrm{K}_{\mathrm{m}}=50.2 \mu \mathrm{M}\right)$ as cofactor, and displayed no catalytic activity with $\mathrm{NADP}^{+}$. The order of the catalytic efficiency of mouse Aldhla1 for aldehyde substrates, as reflected by $\mathrm{V}_{\max } / \mathrm{K}_{\mathrm{m}}$ was $\mathrm{HNE}>\mathrm{MDA}>$ acrolein $>$ trans-2-hexenal $>$ propionaldehyde $>$ acetaldehyde $>$ benzaldehyde. The catalytic efficiency for Aldh2 was highest for acetaldehyde $\left(\mathrm{V}_{\max } / \mathrm{K}_{\mathrm{m}}=22\right)$ followed by propionaldehyde $\left(\mathrm{V}_{\max } / \mathrm{K}_{\mathrm{m}}=13\right)$. This result is 
consistent with the human ALDH2. In Table 1, we observed that benzaldehyde is also a poor substrate for Aldh2. The affinity of Aldh2 for lipid aldehydes was considerably lower than that of Aldh1 a1, acrolein $\left(\mathrm{K}_{\mathrm{m}}=72.5 \mu \mathrm{M}\right)$ and $\mathrm{HNE}\left(\mathrm{K}_{\mathrm{m}}=161 \mu \mathrm{M}\right)$. The rate of metabolism of GSH conjugate of HNE (GS-HNE) by Aldh1a1 and Aldh2 were lower compared with free HNE. Again, Aldhlal showed higher catalytic efficiencies $(\approx 3.6$ fold) for metabolism of GS-HNE compared with Aldh2.

Due to the extremely high $\mathrm{V}_{\max }$ of Aldh3al $(\mu \mathrm{mol} / \mathrm{min} / \mathrm{mg})$ for most aldehyde substrates compared with that of Aldh1al and Aldh2 (nmol/min $/ \mathrm{mg})$, the catalytic efficiency of Aldh3al was higher for most aldehyde substrates except for the lipid aldehydes. In contrast to Aldhla1, mouse Aldh3al exhibited high affinity for aromatic aldehyde, such as benzaldehyde $\left(\mathrm{K}_{\mathrm{m}}=37 \mu \mathrm{M}\right)$ followed by the lipid aldehydes and showed poor affinity for short chain aldehydes, such as propionaldehyde $\left(\mathrm{K}_{\mathrm{m}}=3380 \mu \mathrm{M}\right)$ and acetaldehyde $\left(\mathrm{K}_{\mathrm{m}}=1000 \mu \mathrm{M}\right)$. Aldh3al was capable of metabolizing lipid aldehydes, such as acrolein, $\mathrm{HNE}$ and MDA with low affinity for substrate binding $\left(\mathrm{K}_{\mathrm{m}}\right.$ $>300 \mu \mathrm{M}$ ) compared with Aldh1a1. In addition, both Aldh2 and Aldh3a1 used NAD ${ }^{+}$and $\mathrm{NADP}^{+}$as cofactor for oxidative metabolism of aldehydes, but preferred NAD ${ }^{+}$ $\left(\right.$ Aldh3a1, $\mathrm{V}_{\text {max }} / \mathrm{K}_{\mathrm{m}}=484 ;$ Aldh $\left.2, \mathrm{~V}_{\max } / \mathrm{K}_{\mathrm{m}}=14\right)$ over $\mathrm{NADP}^{+}\left(\mathrm{Aldh} 3 \mathrm{a} 1, \mathrm{~V}_{\max } / \mathrm{K}_{\mathrm{m}}=87\right.$; Aldh2, $\mathrm{V}_{\max } / \mathrm{K}_{\mathrm{m}}=0.6$ ). The catalytic efficiencies for metabolism of acrolein were comparable between Aldh1a1 and Aldh3al $\left(\mathrm{V}_{\max } / \mathrm{K}_{\mathrm{m}}=23\right)$. However, Aldh1a1 exhibited far higher relative affinity for acrolein $\left(\mathrm{K}_{\mathrm{m}}=23.2 \mu \mathrm{M}\right)$ compared with Aldh3a1 $\left(\mathrm{K}_{\mathrm{m}}=\right.$ $464 \mu \mathrm{M})$. Furthermore, the Aldhlal displayed high catalytic efficiency for HNE compared Aldh3al, suggesting that reactive lipid-derived aldehydes were preferred substrates for Aldh1a1. 
Table 1. Kinetic properties of recombinant mouse Aldhs.

Aldh enzyme activity was determined by monitoring the formation of NADH at $340 \mathrm{~nm}$ as previously described by Lindahl and coworkers (Lindahl and Petersen, 1991). Enzyme activities were measured spectrophotometrically at $25^{\circ} \mathrm{C}$ in a reaction mixture containing $1 \mathrm{mMNAD}^{+}$at $\mathrm{pH}$ 7.4. The kinetic parameters were determined using non-linear regression software Kineti77. The values represent the means \pm SD from 3-4 assays. The kinetic parameters for $\mathrm{NAD}^{+}$and $\mathrm{NADP}^{+}$were determined using $1 \mathrm{mM}$ propionaldehyde (Aldh1a1 and Aldh2) or $2.5 \mathrm{mM}$ benzaldehyde (Aldh3a1) as the substrate.

${ }^{\text {a } U n i t ~ i s ~} \mathrm{nmol} / \mathrm{min} / \mathrm{mg}$;

${ }^{\mathrm{b}}$ Unit is $\mu \mathrm{M}$;

${ }^{\mathrm{c}}$ Unit is $\mathrm{ml} / \mathrm{min} / \mathrm{mg}$; and,

${ }^{d}$ Unit is $\mu \mathrm{mol} / \mathrm{min} / \mathrm{mg}$. 
Table 1

\begin{tabular}{|c|c|c|c|c|c|c|c|c|c|}
\hline \multirow[b]{2}{*}{ Substrate } & \multicolumn{3}{|c|}{ Aldh1a1 } & \multicolumn{3}{|c|}{ Aldlo } & \multicolumn{3}{|c|}{ Aldili3a1 } \\
\hline & $V_{\max }{ }^{2}$ & $\mathbf{K}_{\mathbf{m}^{\mathbf{b}}}^{\mathbf{b}}$ & $V_{\max } k_{\mathbf{m}^{c}}$ & $V_{\max }{ }^{a}$ & $\mathbf{K}_{\mathbf{m}} \mathbf{b}$ & $V_{\max } / k_{m}{ }^{c}$ & $V_{\max } d^{d}$ & $\mathbf{K}_{\mathbf{m}} \mathbf{h}$ & $\max ^{\prime} \mathbf{k}_{\mathbf{m}}{ }^{\mathbf{c}}$ \\
\hline Propionaldellyde & $799 \pm 22$ & $141 \pm 30$ & 5.6 & $619 \pm 129$ & $49.4 \pm 13$ & 13 & $24.7 \pm 1.2$ & $3380 \pm 38$ & $?$ \\
\hline Acetaldehyde & $910 \pm 36$ & $202 \pm 17$ & 4.5 & $751 \pm 116$ & $35.7 \pm 14$ & 22 & $32.4 \pm 1.0$ & $1000 \pm 20$ & 32 \\
\hline Benzaldehyde & $256 \pm 14$ & $752 \pm 90$ & 0.3 & $220 \pm 24$ & $1911 \pm 33$ & 0.1 & $28.6 \pm 1.6$ & $37 \pm 6$ & 773 \\
\hline Trans-2-hexenal & $390 \pm 37$ & $31.2 \pm 4.1$ & 12.5 & ND & ND & $N D$ & $28.1 \pm 1.1$ & $236 \pm 40$ & 11 ? \\
\hline HNE & $522 \pm 26$ & $2.4 \pm 0.9$ & 218 & $489 \pm 58$ & $161 \pm 41$ & 3 & $20.5 \pm 3.7$ & $425 \pm 94$ & 69 \\
\hline Acrolein & $527 \pm 22$ & $23.2 \pm 4.3$ & 23 & $543 \pm 36$ & $72.5 \pm 15$ & 7.4 & $10.8 \pm 1.1$ & $464 \pm 85$ & 23 \\
\hline Malondialdelyyde & $408 \pm 15$ & $7 \times 5 \pm 1.2$ & 54 & $\mathrm{ND}$ & ND & ND & $8.3 \pm 0.7$ & $302 \pm 52$ & 27 \\
\hline $\mathrm{NAD}^{+}$ & $751 \pm 7$ & $50.2 \pm 4$ & 15 & $764 \pm 49$ & $56 \pm 6.8$ & 13,6 & $26.9 \pm 59$ & $55.5 \pm 2.3$ & 484 \\
\hline NADP & ND & ND & ND) & $177 \pm 16$ & $281 \pm 37$ & 0.6 & $28 \pm 75$ & $320 \pm 18$ & 87 \\
\hline GS-HNE & $112 \pm 3$ & $32.5 \pm 4.4$ & 3.6 & $161 \pm 26$ & $184 \pm 13$ & 1.0 & $\mathrm{ND}$ & ND & ND) \\
\hline
\end{tabular}




\section{Expression of Aldehyde dehydrogenases in mouse liver and mouse hepatoma cell}

lines

To assess which Aldh isoform plays a prominent role in oxidative metabolism and detoxification of reactive lipid aldehydes in mouse liver, we assessed the mRNA levels of the major Aldhs expressed in mouse liver and Hepa-1c1c7 cells by qRT-PCR. The Aldhla1 gene was highly expressed in mouse liver and Hepa-1c1c7 cells compared with Aldh2, Aldh1b1 and Aldh3a1 (Figure 3). The mRNA levels of Aldh2 were expressed at comparable levels in both mice liver and Hepa-1c1c7 cells. However, Aldh1b1 mRNA expression was higher in Hepa-1c1c7 compared with mouse liver $(\approx 2$-fold). As previously demonstrated, Aldh3a1 mRNA expression was extremely low in mouse liver, but was moderately expressed in Hepa-1c1c7 cells compared with Aldh1a1. Aldh1a1 mRNA levels were 34-fold and 73-fold higher than the Aldh2 mRNA levels in mouse liver and Hepa-1c1c7 cells, respectively. The order of mRNA expression of the major Aldhs involved in lipid aldehyde metabolism in mice liver and Hepa-1c1c7 was Aldhla1 $>$ Aldh2 $>$ Aldh $1 \mathrm{~b} 1>$ Aldh3a1. We concluded from enzyme kinetics and gene expression data that Aldh1al most likely plays a prominent role in the cellular defense against oxidative damage induced by cytotoxic aldehydes in mouse liver. 


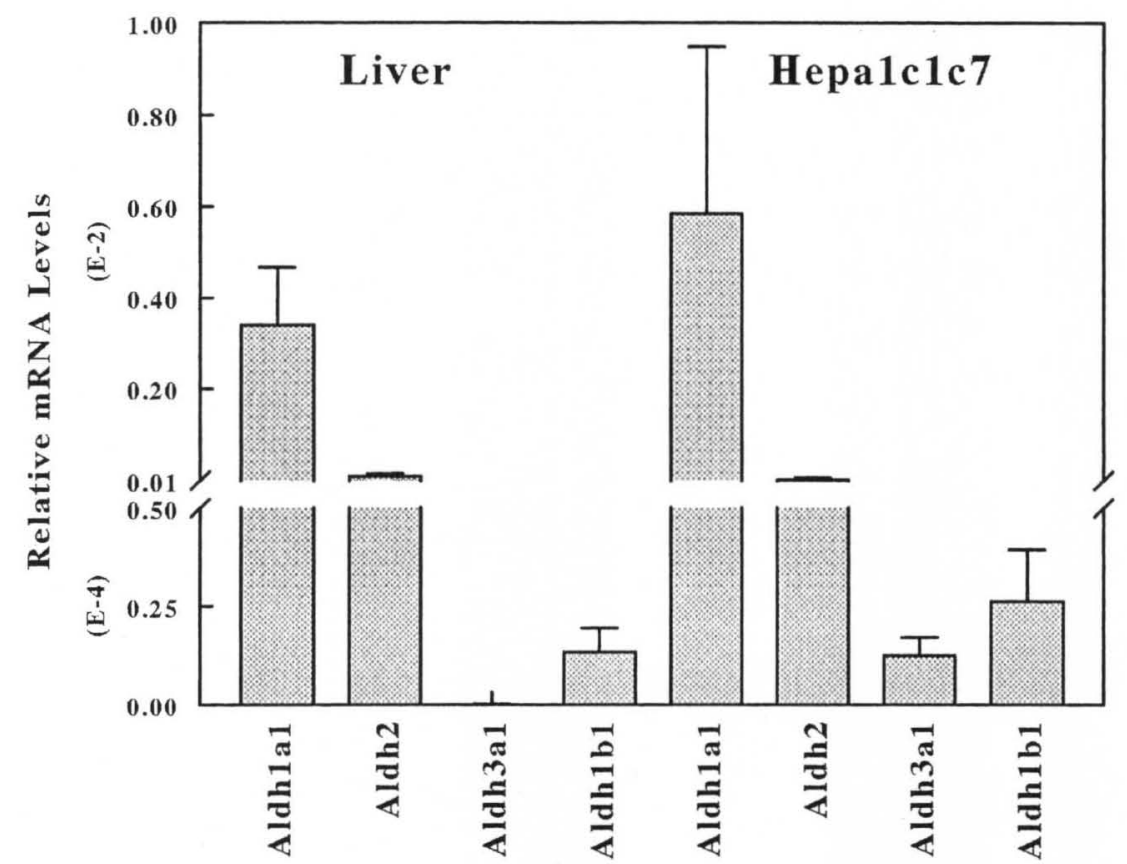

Figure 3. Endogenous levels of aldehyde dehydrogenase gene expression in mouse liver and mouse hepatoma cells. Total RNA extracted from the liver of C57BL/6 mice $(n=5)$ or Hepa-1c1c7 cells was reverse transcribed to cDNA with random hexamer primers using Advantage RT-for-PCR kit. The cDNA was used for analysis of gene expression of Aldh1a1, Aldh2, Aldh1b1 and Aldh3a1 by qRT-PCR. The qRT-PCR experiments were performed in triplicate using cDNA from 5 separate murine liver sample sets. The gene expression levels were normalized to $18 \mathrm{~S}$ rRNA as endogenous control and data were expressed as the mean $\pm \mathrm{SD}$. 


\section{Knockdown of Aldh1a1 gene expression sensitized liver-derived cell lines to acrolein-induced cell death}

To specifically examine the role of Aldh1a1 in cellular protection against reactive lipid-derived aldehydes such as acrolein, we assessed whether knockdown of Aldhla1 gene expression sensitized Hepa-1c1c7 cells to acrolein-induced cell death. Hepa-1c1c7 cells were transfected with either Stealth control or Stealth Aldh1a1-specific siRNA. The effect of knockdown of Aldh1a1 gene expression was assessed by qRT-PCR and Western blot. RNA was isolated from cells $48 \mathrm{~h}$ after transfection and was reverse transcribed to cDNA. The cDNA was then used to assess Aldh1al mRNA expression normalized to $18 \mathrm{~S}$ as endogenous control by qR'T-PCR. In Figure $4 \mathrm{~A}$, we observed $>80 \%$ reduction in Aldh1a1 mRNA levels in stealth Aldhla1-specific siRNA transfected cells compared with untransfected control.

Cell extracts $(40 \mu \mathrm{g})$ prepared from Hepa-1c1c7 cells transfected with Stealth control siRNA or Stealth Aldh1a1-specific siRNA were separated on SDS-PAGE gels and transferred onto nitrocellulose membrane. The membranes were probed with antibodies against rat ALDH1 A1 and GAPDH as endogenous control (Figure 4B). Densitometry analysis of Aldh1al protein levels corrected for GADPH showed $\approx 85 \%$ reduction in Aldh1al protein level in cells transfected with Stealth Aldhla1-specific siRNA compared with untransfected control (Figure 4C). Subsequently, we assessed whether Hepa-1c1c7 cells with knockdown of Aldh1a1 gene exhibit higher sensitivity to acrolein-induced cell death. Twenty four hours after transfection of Hepa-1clc7 cells with either stealth control or Stealth Aldh1al siRNA, cells were exposed to a range of acrolein concentrations $(0-100 \mu \mathrm{M})$ for $24 \mathrm{~h}$ and cell viability was examined by MTT 
assay. Figure 3D showed that Hepa-1clc7 cells exhibit higher sensitivity to acrolein, with $25 \mu \mathrm{M}$ and higher concentrations producing concentration-dependent decrease in cell viability. We also observed a comparable dose-dependent decrease in viability between untransfected and control siRNA transfected cells. However, knockdown of Aldh1al gene expression resulted in marked decreased in cell viability compared with WT cells. The half maximal effective concentrations $\left(\mathrm{EC}_{50}\right)$ of acrolein for cells with low expression of Aldh1a1 ( $\left.\mathrm{EC}_{50} \approx 22 \mu \mathrm{M}\right)$ was significantly reduced compared with that of the untransfected and control siRNA transfected cells $\left(\mathrm{EC}_{50} \approx 35 \mu \mathrm{M}\right)$. These results indicate that cells with low Aldhlal expression are highly vulnerable to acrolein-induced cell death, suggesting that Aldh1a1 protects cells from reactive aldehydes-induced cell death by enhanced metabolism of these reactive species. 
Figure 4. Hepa-1c1c7 cells with knockdown of Aldh1a1 gene expression by stealth specific siRNA are highly sensitive to acrolein-induced cell death compared with control cells. (A-C) Stealth specific siRNA mediated knockdown of Aldh1a1 gene in Hepa-1c1c7 cells. Hepa-1c1c7 cells were transfected with either $150 \mathrm{pmol}(50 \mathrm{nM})$ Stealth control (siControl) or Stealth Aldh1a1-specific siRNA (siAldh1a1) using Lipofectamine RNAiMAX. (A) qRT-PCR analysis of Aldh1a1 gene expression in various Hepa-1c1c7 cells. The mRNA expression of Aldh1a1 was examined by qRT-PCR and experiments were performed in triplicate using cDNA sample from independent RNA set. The gene expression levels were normalized to $18 \mathrm{~S}$ rRNA as an endogenous control and data were expressed as fold over WT. (B) Western blot analysis of Aldh1a1 expression in Hepa$1 \mathrm{c} 1 \mathrm{c} 7$ cells. Cell extracts prepared from WT, siControl and siAldh1a1 cells were separated on 4-12\% SDS-PAGE gel and transferred onto nitrocellulose membrane. Membranes were probed with antibodies against ALDH1A1. Membranes were stripped and then reprobed with antibodies against GAPDH. The Western blot experiment was performed in triplicate and the figure is a representative blot. (C) Densitometry analysis of Aldh1a1 protein levels normalized to GAPDH in WT, siControl and siAldh1al Hepa$1 \mathrm{c} 1 \mathrm{c} 7$ cells. (D) Knockdown of Aldh1al sensitizes Hepa-1c1c7 cells to acrolein treatment. Twenty four $\mathrm{h}$ after transfection with Stealth siRNA control or Stealth Aldh1a1-specific siRNA, cells were treated with increasing concentrations of acrolein for $24 \mathrm{~h}$ and cell viability assessed by MTT $(0.2 \mathrm{mg} / \mathrm{ml}$ for $2 \mathrm{~h})$ at $570 \mathrm{~nm}$ as described in Materials and Methods. The half maximal effective concentrations $\left(\mathrm{EC}_{50}\right)$ were as follows: WT untransfected cells $(35.61 \pm 1.64 \mu \mathrm{M})$, Stealth control siRNA transfected cells $(35.72 \pm 1.62 \mu \mathrm{M})$ and Stealth Aldh1a1-specific siRNA transfected cells $(22.92 \pm$ 
$0.32 \mu \mathrm{M})$. The values are expressed as the mean \pm S.E. and the $\mathrm{EC}_{50}$ for SiAldh1a1 ablated cells were statistically different that of control or SiControl cells ( $\mathrm{p} \leq 0.05$ ).

Figure 4

A.

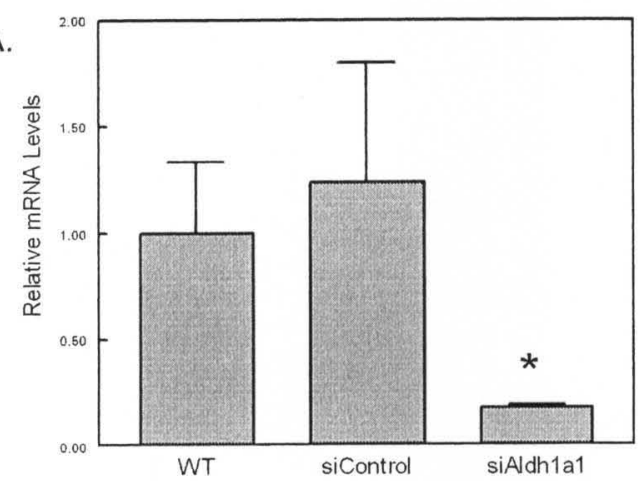

B.

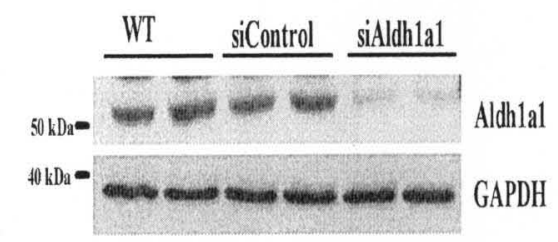

C.

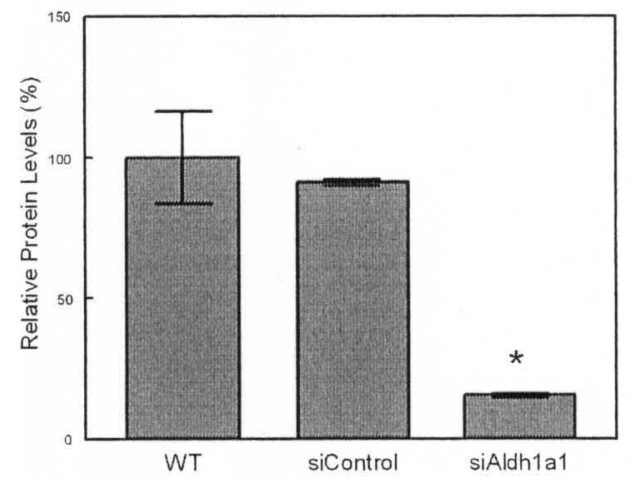

D.

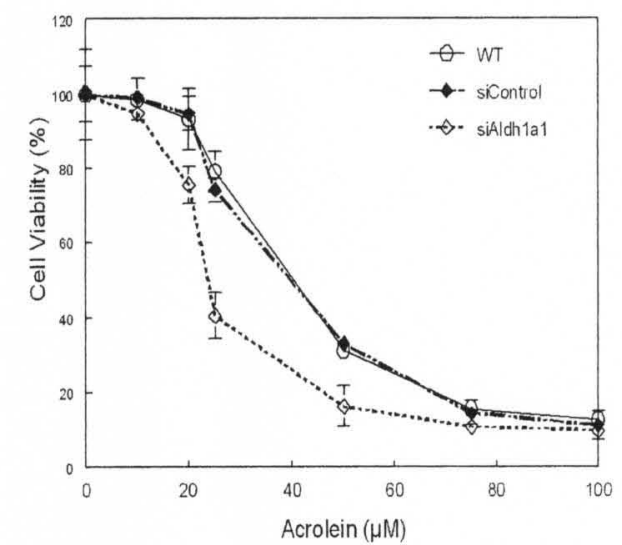




\section{Increased acrolein-protein adducts in Hepa-1c1c7 cells with siRNA silencing of}

\section{Aldh1a1 gene expression}

Acrolein and other highly reactive $\alpha, \beta$-unsaturated aldehydes readily alkylate proteins forming Michael addition adducts with a free carbonyl group. It is now known that protein carbonylation by reactive aldehydes precedes the toxic process leading to cell death (Burcham and Fontaine, 2001). We examined whether acrolein-protein adducts are increased in cells with knockdown of Aldh1a1 after acrolein treatment. Hepa-1c1c7 cells were transfected with either stealth control siRNA (siControl) or Aldh1al specific stealth siRNA (siAldh1a1). After transfection, cells were exposed to either $10 \mu \mathrm{M}$ or $25 \mu \mathrm{M}$ acrolein for $24 \mathrm{~h}$. Lysates prepared from cells were separated on SDS-PAGE and transferred onto nitrocellulose membrane. The membrane was probed with antibodies against acrolein-protein adducts and GAPDH (Figure 5A). The level of acrolein-protein adducts of individual proteins was analyzed by densitometry corrected for GADPH using UN-SCAN-IT gel 6.1 software (Silk Scientific Inc., Orem, Utah). Three protein bands labeled P1, P2 and P3 were chosen for analysis of acrolein-protein adducts accumulation in the siControl and siAldh1al cells following exposure to acrolein $(10 \mu \mathrm{M}$ and $25 \mu \mathrm{M})$ (Figure 5B). Densitometry analysis showed that acrolein-protein adducts content of P1 and P3 continued to increase in both siControl and siAldhal-treated cells up to $25 \mu \mathrm{M}$ acrolein. We also noticed accumulation of endogenous acrolein-protein adducts for protein P1, P2 and P3 in siAldhlal-treated cells compared with siControl cells. In protein 1 and 3 (P1 and P3), the level of acrolein-protein adducts were increased in Aldh1a1specific Stealth siRNA transfected cells compared with control siRNA-transfected cells after treatment with $10 \mu \mathrm{M}$ acrolein. 
Figure 5. The levels of acrolein-protein adducts in response to acrolein treatment were increased in Hepa-1c1c7 cells with knockdown of Aldh1a1 gene compared with control siRNA-transfected control cells (A) Western blot analysis of acrolein-protein adducts in Hepa-1c1c7 cells transfected with either stealth control siRNA or Aldh1a1-specific siRNA and exposed to acrolein. Lanes 1-2: untreated cells, Lanes 3-4: cells were treated for $24 \mathrm{~h}$ with $10 \mu \mathrm{M}$ acrolein. Lanes 5-6: cells exposed to $25 \mu \mathrm{M}$ acrolein for $24 \mathrm{~h}$. Western blot experiments were performed in triplicate and the figure is a representative blot. (B) Densitometry analysis of the levels of individual protein-acrolein adducts in siControl (Stealth control siRNA transfected cells) and siAldhla1 (Stealth Aldhla1 specific siRNA-transfected cells) in response to $10 \mu \mathrm{M}$ and $25 \mu \mathrm{M}$ acrolein. Samples were analyzed by 2 -tailed $t$-test and values of $\mathrm{p}<0.05$ were considered to be statistically significant. *, significantly different from siControls; and \#, indicates significantly different from untreated siControl. 
Figure 5

A.

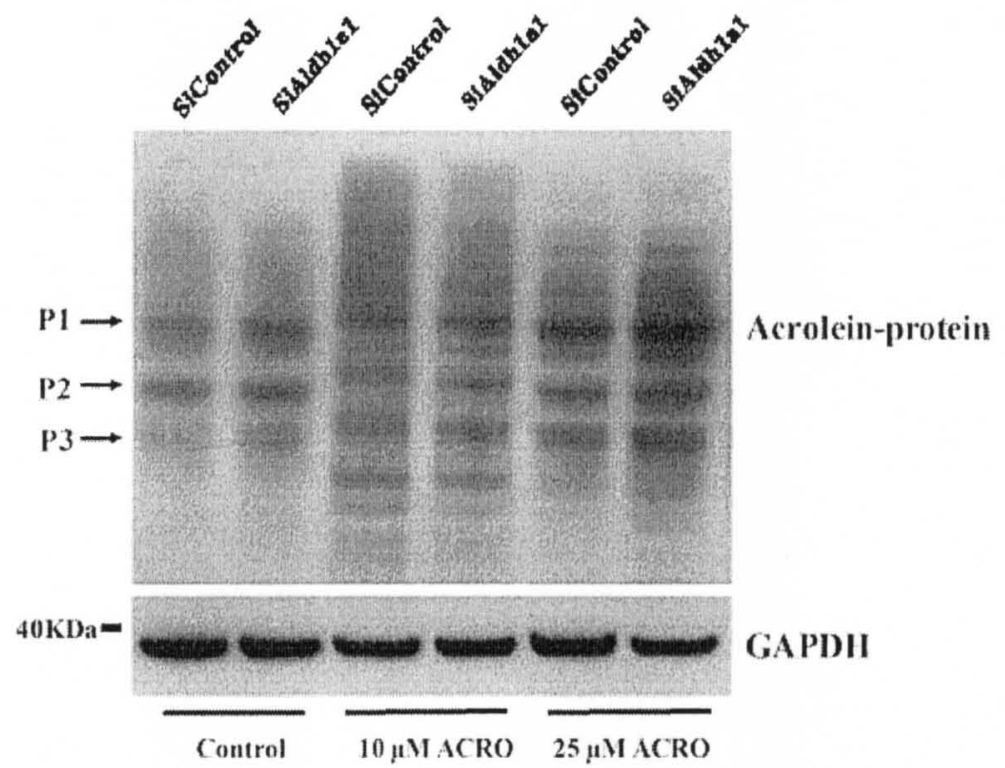

B.

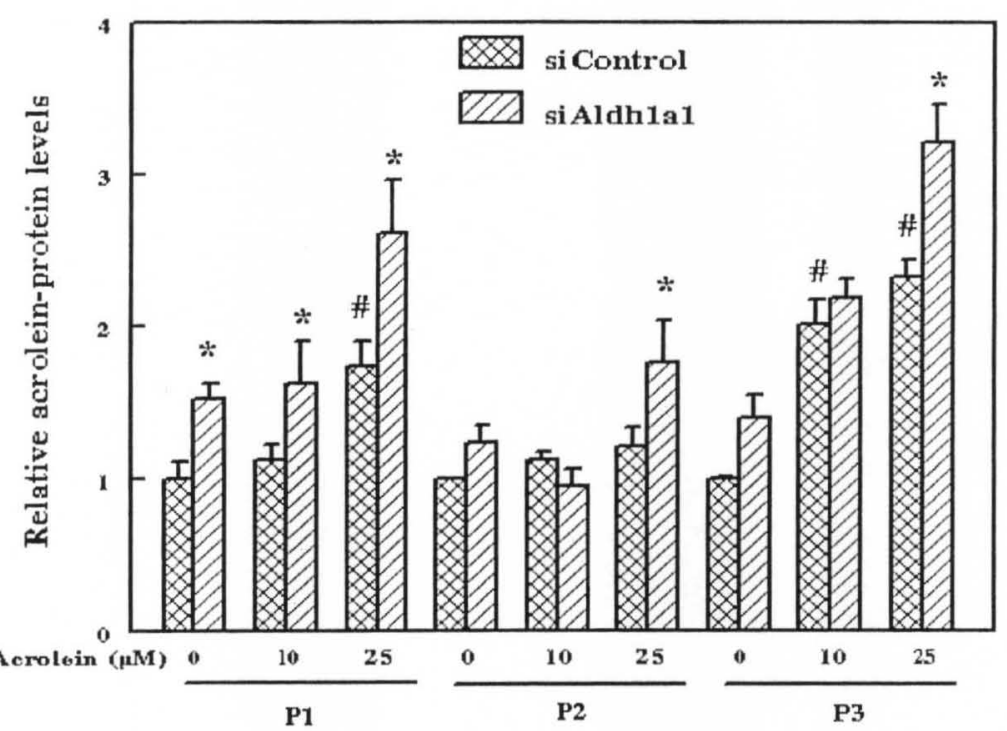


However, for P1, P2 and P3, we observed elevated levels of acrolein-protein adducts in siAldh1a1 cells compared to siControl cells at $25 \mu \mathrm{M}$ acrolein treatment. These results indicate that when Aldh1al protein levels are low, the impaired acrolein metabolism and detoxification led to accumulation of acrolein-protein adducts.

\section{Increased acrolein-induced Caspase 3 activation and apoptosis in Hepa1c1c7 cells after knockdown of Aldh1a1 gene expression}

In addition to protein-acrolein adducts accumulation and cell viability, we investigated whether knockdown of Aldh1a1 sensitized cells to acrolein-induced caspase 3 activation, a marker of apoptosis. Reactive lipid aldehydes are known to induce oxidative stress by depletion of GSH (Ellis, 2007). Oxidative stress and acrolein-protein or HNE-protein adducts have been implicated in induction of apoptosis (Tanel and Averill-Bates, 2007; Xiao et al., 2003). Previous studies demonstrated that HNE induces activation of caspase 3 in human lens epithelia cells (HLECs), human leukemia cells (HL-60) and neuroblastoma cell line (SH-SY5Y) (Cheng et al., 2001; Choudhary et al., 2002;Zhang et al., 2010). Inactive procaspase 3 (32 KDa) zymogen is activated by proteolytic cleavage adjacent to Asp175 into activated 17- and 19-KDa fragments. Thus, the activation of caspase 3 is monitored by Western blot analysis of the cleaved fragments. To examine whether Aldh1a1 can protect cells against acrolein-induced apoptosis, we transfected Hepa-1c1c7 cells with Stealth Aldh1a1-specific siRNA (siAldhlal) or Stealth control siRNA (siControl) for $24 \mathrm{~h}$ and cells were subsequently exposed to $25 \mu \mathrm{M}$ acrolein for $24 \mathrm{~h}$. Cell extracts separated by SDS-PAGE gels were transferred onto nitrocellulose membrane and probed for cleaved caspase 3 fragments, 
Figure 6. Transient knockdown of Aldhlal gene expression sensitizes Hepa-1clc7 cells to acrolein-induced caspase 3 activation and apoptosis. (A) Western blot analysis of acrolein-induced caspase 3 activation. Hepa-1clc 7 cells were transfected with Stealth Aldh1a1-specific siRNA (siAldh1a1) or Stealth control siRNA (siControl) for $24 \mathrm{~h}$ and cells were subsequently exposed to $25 \mu \mathrm{M}$ acrolein for $24 \mathrm{~h}$. Cell extracts prepared with 1X RIPA buffer were separated on SDS-PAGE gel and transferred onto nitrocellulose membranes. The membranes were probed for cleaved caspase 3 with anti-cleaved caspase 3 antibodies and caspase 3 with anti-caspase 3 antibodies. Anti-cleaved caspase 3 (Asp175) antibody detects levels of large fragment (17/19 KDa) of activated caspase 3 resulting from cleavage adjacent to Asp175. Western blot experiment was performed in triplicate and the figure is a representative blot. Densitometry was normalized to GAPDH of procaspase 3 (B) and cleaved caspase 3 (17 and $19 \mathrm{KDa}$ fragments) (C) in WT, siControl- or siAldhlal-treated Hepa-1c1c7 cells exposed to $25 \mu \mathrm{M}$ acrolein. Positive $(+)$ control for caspase 3 cleavage was extracts from cytochrome $c$-treated Jurkat cells (\#9663) and negative (-) control was extract from untreated Jurkat cells. The results were analyzed by Student's - test and values of $\mathrm{p}<0.05$ were considered to be statistically significant. *, significantly different from WT ( $\mathrm{p}<0.05 ; 2$-tailed t-test). 
Figure 6
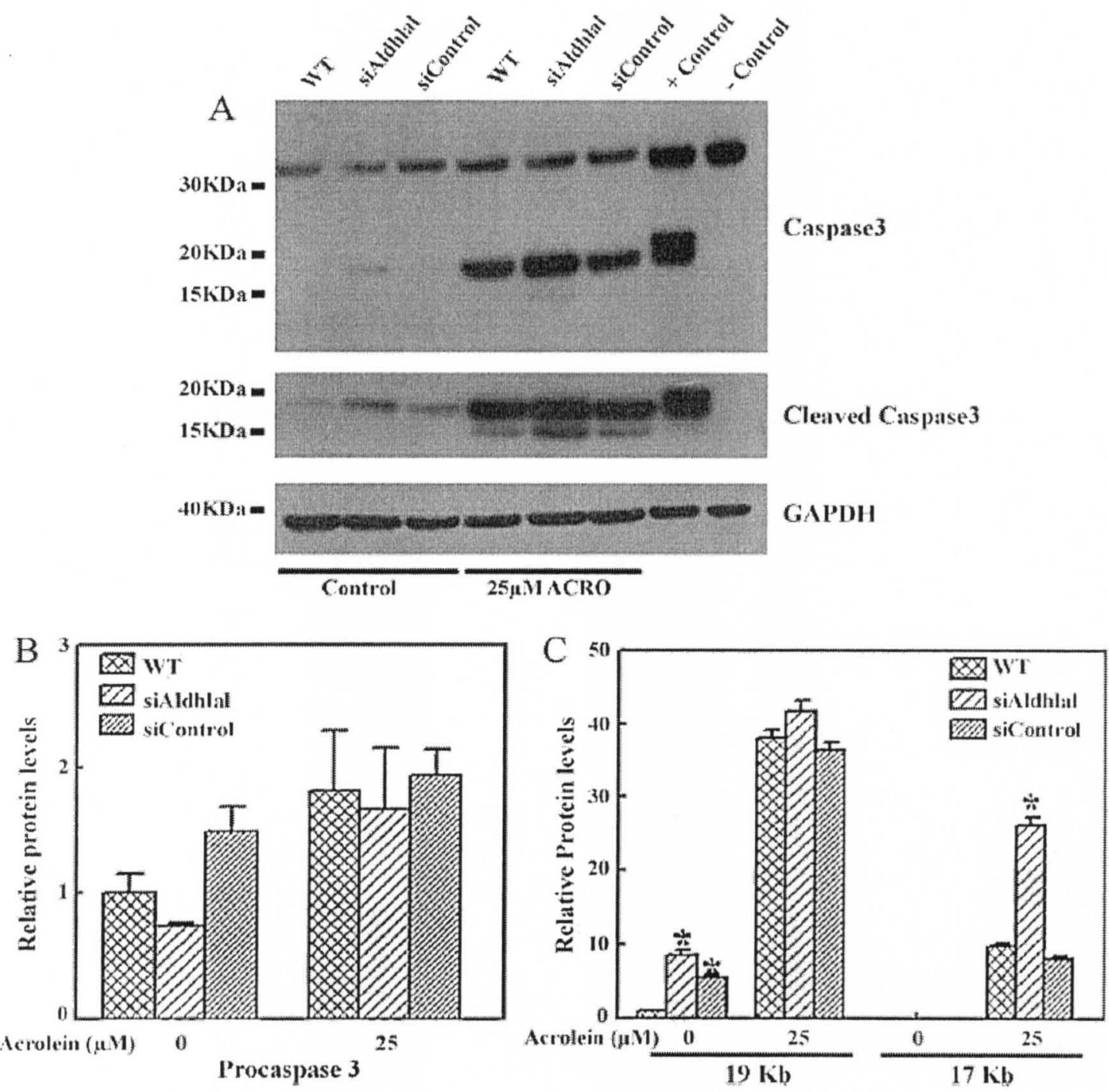
caspase 3 and GADPH as endogenous control (Figure 6A). The levels of procaspase 3 (Figure 6B) and cleaved caspase 3 (17 and $19 \mathrm{KDa}$ fragments) (Figure 6C) in untransfected cell (WT), siControl- and siAldh1a1-treated Hepalc1c7 cells exposed to 25 $\mu \mathrm{M}$ acrolein were assessed by densitometry corrected for GADPH using UN-SCAN-IT gel 6.1 software. The levels of procaspase 3 were moderately increased in WT and siAldh1a1 cells after acrolein treatment. However, procaspase 3 protein levels were slightly decreased in siAldh1a1 cells in the absence of acrolein treatment. As expected, the levels of the 17 and $19 \mathrm{KDa}$ fragments of caspase 3 (active) were robustly increased $(\approx 40$-fold $)$ when cells were exposed to acrolein compared to untreated WT cells or control siRNA transfected cells. The $19 \mathrm{KDa}$ fragment was moderately increased in siAldh1al cells compared with WT and siControl cells after acrolein $(25 \mu \mathrm{M})$ exposure (Figure 6C). However, we observed elevated levels ( $\approx 3$-fold) of the $17 \mathrm{KDa}$ fragment in siAldh1al cells compared with WT and siControl cells after acrolein $(25 \mu \mathrm{M})$ treatment. These results indicate that Aldh1al knockdown cells exhibit high sensitivity to acroleininduced caspase 3 initiated apoptosis. We can conclude from these data that Aldhlal by enhanced detoxification and metabolism of acrolein protects cells against apoptosis induced by acrolein.

\section{DISCUSSION}

\section{Comparison of Substrate Specificity for murine Aldhs}

The relative contribution of Aldh isozymes in protection against aldehyde toxicity is controversial. While earlier studies indicated that ALDH3A1 but not ALDH1A1 protects against cytotoxic lipid derived-aldehydes, most recent studies in eye lens of 
Aldh 1 a ${ }^{-1 /}$ mice showed that ALDH1A1 can protect against these reactive aldehydes (Choudhary et al., 2005; Townsend et al., 2001). The contribution of the different isozymes of Aldh to oxidation of lipid derived-aldehydes has not been examined in the liver, even though the liver is the major site for metabolism and biotransformation of foreign compounds. In this study, we sought to clearly define which Aldh isoforms play a major role in oxidation and detoxification of cytototic lipid-derived aldehydes in mouse liver by enzyme kinetics and gene expression pattern. Having obtained purified recombinant murine Aldhs expressed in E. coli, we performed enzyme kinetic analysis to compare their relative capacity for clearance of various aldehydes. As seen in Table 1, the catalytic efficiencies can best be compared using the $\mathrm{V}_{\max } / \mathrm{K}_{\mathrm{m}}$ parameters. Aldh3al displays highest preference for aromatic aldehyde substrates and utilizes both $\mathrm{NAD}^{+}$and $\mathrm{NADP}^{+}$as oxidizing pyridine nucleotide cofactors with preference for $\mathrm{NAD}^{+}$. Although Aldh3A1 displays 20-112-fold higher $\mathrm{V}_{\max }$ values for most aldehyde substrates, the $\mathrm{V}_{\max } / \mathrm{K}_{\mathrm{m}}$ parameters demonstrate that Aldh1al is a better catalyst than Aldh3a1 for oxidative clearance of $\alpha, \beta$-unsaturated aldehydes. The human and murine Aldh1a1 proteins exhibit similar $\mathrm{V}_{\max } / \mathrm{K}_{\mathrm{m}}$ parameters with some differences (Xiao et al., 2009). The human ALDH2 (Marchitti et al., 2008) and ALDH1B1 (Stagos et al., 2010) proteins displayed significantly lower $V_{\max } / K_{m}$ parameters for the lipid-derived aldehydes than Aldh1a1. The substrate specificity of recombinant Aldhs for GS-conjugates of HNE indicates that GS-HNE is a good substrate for Aldh1a1 and Aldh2. However, both enzymes display a $\mathrm{K}_{\mathrm{m}}$ value for GS-HNE that is much higher than the free HNE itself, suggesting that free HNE is a better substrate for these enzymes than its GSH conjugate. Aldh1a1 showed higher catalytic efficiencies $(\approx 3.6$ fold) for metabolism of GS-HNE 
compared with Aldh2. Kinetic studies also demonstrate that GS-HNE is not a substrate of Aldh3al. The conclusion taken from the data in this report and the literature is that the catalytic efficiency for lipid aldehyde oxidation by Aldh1a1 is the highest reported, suggesting its potential importance for metabolism of these toxic compounds.

\section{Aldh1a1 is highly expressed in mouse liver and Hepa-1c1c7 cells}

In Figure 3, we showed that Aldh1a1 is highly expressed in the livers of male C57BL/6 mice and in mouse hepatoma Hepa-1c1c cells relative to other Aldh enzymes. The levels of Aldh1a1 mRNA were about 34-fold higher than that of the next more abundant mRNA, Aldh2. There was much less Aldh1b1 and Aldh3A1 mRNA expression in either liver or Hepa-1c1c cells. This observation is supported by the data in Chapter III, which demonstrate that acrolein caused a 3-fold induction of Aldh1a1 mRNA in male mouse livers, while other forms of Aldh were not induced by treatment with acrolein, providing a mechanism for the liver cell to respond to toxic aldehydes. In addition, it is known that hepatic Aldh1a1 is also induced in animals treated with butylated hydroxyanisole (Alnouti and Klaassen, 2008) or phenobarbital (Deitrich et al., 1977). These compounds are known activators of transcription factors such as activator protein 1 (AP1), nuclear factor-erythroid 2 related factor 2 (Nrf2), and constitutive androstane receptor (CAR). These observations clearly demonstrate that mouse Aldh1a1 and probably the human ortholog (ALDH1A1) are major protective enzymes against the products of lipid peroxidation and other toxic aldehydes in the environment. 


\section{Ablation of Aldh1a1 gene expression increases acrolein-induced cell death, acrolein}

protein adducts, and caspase cleavage

As a method of assessing the role of specific gene products in toxicological processes, an in vitro method using gene ablation allows one to test the role of specific proteins in response to toxic agents. Because Hepa-1c1c7 cells appeared to recapitulate the spectrum of Aldhs expressed in mouse liver, we chose this cell system to test whether ablation of Aldh1a1 protein and enzyme activity increases the sensitivity of these cells to acrolein (Figure 4). Using Stealth ${ }^{\mathrm{TM}}$ siRNA knockdown technology, we documented that transfection of Aldh1a1-specific siRNA suppresses the levels of Aldh1al mRNA and protein in Hepalclc cells (Figure 4A-C). We then challenged these cells with increasing concentrations of acrolein to assess the relative sensitivity to acrolein toxicity (Figure 4D). While the control siRNA had no effect on acrolein toxicity curve as measured with MTT-dependent cell viability, transfection of Hepa-1c1c7 cells with Aldh1a1-specific siRNA caused a shift to the left in the acrolein toxicity curve, clearly demonstrating that cells with Aldhla1 ablation were statistically more sensitive to acrolein toxicity than control siRNA transfected or untransfected cells. These results provide evidence of an important protective role for Aldh1a1 against $\alpha, \beta$-unsaturated aldehydes in mouse liver.

Previous experiments demonstrate that treatment of cells with acrolein led to increased acrolein-protein adducts and enhanced caspase 3 activation resulting in proteolytic cleavage of PARP (Burcham and Fontaine, 2001;Tanel and Averill-Bates, 2007). We measured the levels of acrolein-protein adduct in cells transfected with Aldh1a1-specific siRNA and noted that the levels of acrolein-protein adducts (P1, P2, P3) were significantly increased relative to control siRNA transfected cells (Figures 5A, B). 
In addition to $\mathrm{P} 1, \mathrm{P} 2$, and $\mathrm{P} 3$, low concentration $(10 \mu \mathrm{M})$ of acrolein resulted in a larger amount of lower molecular weight acrolein-linked proteins that was not seen at higher concentrations, most likely due to less extensive cross-linking present in P1, P2, and P3. This could be due to the use of non-reducing Western blot At higher acrolein concentrations, these lower molecular weight protein-adduct species decreased in amount, while the higher molecular weight forms increased significantly as a function of acrolein concentration. In addition, caspase 3 cleavage to a $17 \mathrm{KDa}$ fragment was strikingly increased in siAldhlal transfected Hepa-1clc7 cells exposed to $25 \mu \mathrm{M}$ acrolein, relative to untransfected or siControl transfected cells (Figure 6). These results clearly demonstrate that the pro-apoptotic changes caused by acrolein and levels of acrolein-protein adducts are increased under conditions of Aldhlal depletion. In summary, we presented evidence that Aldh1a1 plays a major role in cellular defense against oxidative damage induced by reactive lipid aldehydes in mouse liver. Aldh1al exhibits far higher affinity for acrolein and HNE compared with Aldh2 or Aldh3a1. The endogenous Aldh1al gene was highly expressed in mouse liver and liverderived cell lines compared with Aldh2 and Aldh3a1. The knockdown of Aldh1al expression by siRNA caused Hepa-1c1c7 cells to be more sensitive to acrolein-induced cell death and resulted in increased accumulation of acrolein-protein adducts and caspase 3 activation. Thus, mechanisms to induce Aldhla1 gene expression may provide a useful rationale for therapeutic protection against oxidative stress-induced pathologies. 


\section{CHAPTER III}

\section{MOLECULAR MECHANISMS UNDERLYING TRANSCRIPTIONAL REGULATION OF MOUSE HEPATIC ALDEHYDE DEHYDROGENASE 1A1 BY ELECTROPHILES}

\section{INTRODUCTION}

The ALDH gene superfamily encodes enzymes that catalyze the NAD $(P)^{+}-$ dependent oxidation of aldehydes generated from a wide variety of endogenous and exogenous processes to their corresponding carboxylic acids. There are 555 genes encoding ALDH proteins with 172 of these genes found in eukaryotes (Sophos and Vasiliou, 2003). At present, 19 functional ALDH genes have been identified in the human genome. Among the Aldh isoforms, the cytosolic mouse Aldh1a1 plays a critical role in oxidative metabolism of highly toxic lipid aldehydes and protects liver-derived cells from the toxic effects of lipid aldehydes (Makia et al., 2011). Lipid peroxidation generates many aldehydes as by products, among which acrolein and HNE are presumably the most toxic and harmful. These aldehydes are implicated in the pathogenesis of oxidative stress-induced pathologies, such steatohepatitis, atherosclerosis, myocardial ischemia/reperfusion injury, cataract and cancer.

Murine Aldhlal gene, previously known as Retinaldehyde dehydrogenase 1 (Raldh1 or Ahd-2) is found on chromosome 19 and spans $45 \mathrm{~kb}$ in length (Hsu et al., 1999; Hsu et al., 2000). It contains 13 exons and encodes a protein of $\approx 501$ amino acids 
with a molecular weight of $\approx 55 \mathrm{KDa}$. Mouse Aldh1 gene shares $96 \%$ and $87 \%$ of its amino acid sequence with rat ALDH1 and the human ALDH1 respectively, indicating that it is the mouse homologue of the human ALDH1 gene (Hsu et al., 1999). The tissue distribution of mouse Aldhlal gene is similar to that of human $A L D H I$ gene. It is highly expressed in the liver, lung, kidney, eye lens, gonads and retina (Marchitti et al., 2008; Vasiliou V et al., 2004). Increased expression of Aldhlal gene in the liver and lens has been postulated as a mechanism to protect these organs against cytotoxic aldehydesinduced cellular damage. Thus, an understanding of the molecular regulation of Aldh1a1 gene expression is of importance in ameliorating the toxic effects of carbonyl compounds and in preventing oxidative stress-induced pathologies.

Although the human and mouse Aldhlal gene have been cloned and the promoter region characterized, little is known about the molecular mechanisms that regulate the expression of this gene in mouse liver (Elizondo et al., 2009;Hsu et al., 1999). An understanding of the factors controlling Aldhlal gene expression may assist efforts to control the progression of oxidative stress-mediated diseases. The expression of Aldh1al gene is regulated by a wide variety of endogenous and exogenous stimuli. Aldh1a1 expression is reported to be enhanced by activators of $\mathrm{Nrf} 2$ (Hu et al., 2006; Lee et al., 2003;Reddy et al., 2007; Thimmulappa et al., 2002). Recent studies indicate that oxidative stress-mediated activation of Nrf2 induces the expression of antioxidant and electrophiles detoxifying genes including Aldhlal in an in vivo mice model of renal ischemia/reperfusion injury (Leonard et al., 2006). Furthermore, the basal expression of Aldhlal gene was significantly reduced in $\mathrm{Nrf}^{-1-}$ mice compared with wild type (WT) mice (Lamle et al., 2008), suggesting that the basal expression of Aldhlal may be 
regulated by Nrf2. However, the signaling pathway involved in electrophile-induced expression of Aldhlal gene is not completely understood. It is not yet known whether Aldh1a1 gene is a direct target of $\mathrm{Nrf2}$, since most activators of $\mathrm{Nrf} 2$ are also known inducers of the AP-1 proteins.

There is increasing evidence that acrolein and related aldehydes, such as HNE and crotonaldehyde at non-toxic concentrations, are important signaling molecules and can induce the expression of cytoprotective genes encoding antioxidant and electrophile detoxification enzymes (Liu et al., 2010; Ranganna et al., 2002;Tirumalai et al., 2002). Interestingly, many of these cytoprotective genes are involved in metabolism and detoxification of reactive aldehydes. The signaling pathways involved in reactive aldehydes-induced expression of cytoprotective genes are not completely understood. However, reactive aldehydes are known activators of redox-responsive transcription factors, namely nuclear factor-erythroid 2 related factor 2 (Nrf2), nuclear factor kappa B (NF-kB) and members of the activator protein 1 (AP-1) family (Kwak et al., 2003;Park et al., 2005;Pugazhenthi et al., 2006;Ranganna et al., 2002;Tirumalai et al., 2002;Wu et al., 2006; Zhang and Forman, 2008).

Nrf2 and AP-1 transcription factors are hepatoprotective against oxidative and electrophilic stress by transcriptional up-regulation of antioxidant and electrophile detoxifying genes. The activation of $\mathrm{Nrf2}$ by electrophiles is known to disrupt its association with Keap-1 leading to Nrf2 translocation and nuclear accumulation. In the nucleus, Nrf2 heterodimerizes with the small Maf proteins and binding to specific response elements termed $\mathrm{ARE} / \mathrm{EpRE}$ coordinates expression of hepatoprotective genes. AP-1 proteins are also a binding partner for Nrf2. However, AP-1 transcription factors 
preferably modulate gene expression as either homodimers of $j u n$ family or heterodimers with members of the fos family. There is now compelling evidence to indicate that AP-1 proteins are hepatoprotective especially against oxidative and electrophilic stress. AP-1 proteins were shown to be cytoprotective against apoptosis induced by UV irradiation or TNF $\alpha$ exposure (Weitzman et al., 2000;Lamb et al., 2003). Moreover, JunD regulates the expression of human ferritin $\mathrm{H}$ genes which is protective against oxidative stressmediated cytotoxicity (Tsuji, 2005). Hasselblatt et al. demonstrate that hepatocyte survival during acute hepatitis was dependent on c-Jun/AP-1-induced expression of inducible nitric oxide synthase (nos2) gene (Hasselblatt et al., 2007). However, there has been no report on the role of the redox-sensitive transcription factors, AP-1 and Nrf2 in the regulation of Aldhs and detoxification of lipid peroxidation.

The purpose of this study was to investigate whether well-known electrophiles modulate the expression of Aldh1a1 in mouse liver and to elucidate the signaling pathways involved. Gene expression profiles in the liver by acrolein and BHA were analyzed by microarray to examine whether acrolein or BHA exposure up-regulates the expression of genes encoding antioxidant or electrophile detoxification enzymes in mice liver (Amunom, Makia and Prough, unpublished). Mice were administered AIN76A (control) diet, diet containing $0.45 \% \mathrm{BHA}$ or $5 \mathrm{mg} / \mathrm{kg}$ acrolein by gavage for 7 days. The expression of genes encoding several electrophile detoxifying enzymes such as Gstm1, Gsta3, Gstp, Gstz1, Akr1c13, Akr1c19, Akr1c20 and Akrla4 was specifically elevated ( $\geq 2$-fold), indicating a detoxification and antioxidant response. The elevation of Aldh1a1 gene was noticeable with a 2- to 3-fold increase by both electrophiles. These results 
support a model in which high acrolein levels in cells stimulate Aldh1a1 gene expression, thereby enhancing acrolein metabolism.

In vivo, BHA is oxidatively demethylated by cytochrome P450 to tertbutylhydroquinone ( $t$-BHQ) with an oxidizable 1, 4-diphenolic structure. The resultant $t$ BHQ can undergo redox cycling by two-electron oxidation to tert-butylbenzoquinone (tBQ) generating semiquinone radicals and ROS (Abiko et al., 2011;Nakamura et al., 2003; Pinkus et al., 1996). Even though, BHA is a known activator of Nrf2, studies by Pinkus et al demonstrated that BHA-induced expression of GST-Ya gene is mediated by induction of AP-1 activity (Pinkus et al., 1996). Compelling evidence was presented showing that the induction of AP- 1 transcriptional activity by BHA or $t$-BHQ is due to quinone-mediated generation of oxygen radicals and oxidative stress. However, the metabolite of $t$-BHQ, similarly to acrolein, can act as an electrophile and reacts with intracellular nucleophiles including protein thiol and GSH by Michael addition reactions, resulting in protein alkylation and GSH depletion. GSH-tBHQ conjugates have been detected in cells exposed to $t$-BHQ (Nakamura et al., 2003). As an electrophile, $t$-BHQ can also cause dissociation of the Keap1-Nrf2 complex through covalent modifications of cysteine residues $\left(\mathrm{C}^{257}, \mathrm{C}^{273}, \mathrm{C}^{288}\right.$ and $\mathrm{C}^{297}$ ) on Keap-1, resulting in nuclear accumulation of Nrf2 (Nguyen et al., 2004;Rushmore and Pickett, 1991).

In this chapter, we focus on electrophile-mediated regulation of Aldhlal in mouse liver. The mRNA expression and activity of Aldh1a1 in liver from mice treated with BHA or acrolein were measured by qRT-PCR and Aldh activity assay, respectively. Second, we examined the signaling pathway involved in BHA- or acrolein-mediated induction of Aldhla1 gene expression. Since the major function of Aldhla1 is to detoxify 
highly toxic lipid aldehydes, the induction of Aldh1 a1 gene in mice liver might be a mechanism to alleviate toxicity associated with lipid peroxidation and could represent a useful strategy for preventing oxidative stress-associated diseases.

\section{EXPERIMENTAL DESIGN and METHODS}

\section{Chemicals}

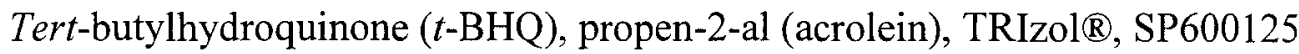
(JNK inhibitor), PD98059 (MEK inhibitor) and okadaic acid (phosphatase inhibitor) were purchased from Sigma-Aldrich Company, Inc. (St Louis, MO). AIN-76A diet and AIN76A diet containing butylated hydroxyanisole (BHA) were obtained from Purina Laboratories. Anti-glyceraldehyde-3-phosphate dehydrogenase (GAPDH) antibody, clone 6C5 (MAB374) was purchased from Millipore (Temecula, CA). Rabbit polyclonal antibodies against c-Jun (N; sc-45), Jun D (329; sc-74), Nrf2 (C-20; sc-722), JNK (FL; sc-572) and pJNK (G-7; sc-6254), horseradish peroxidase-conjugated goat anti-rabbit (Sc-2004) and goat anti-mouse (Sc-2005) were purchased from Santa Cruz Biotechnology, Inc (Santa Cruz, CA). Phospho c-Jun (Ser63 II) antibody (\#9261) was obtained from Cell Signaling Technology (Danvers, MA).

\section{Animals Genotyping and Treatment}

Wild type (WT) and Nrf2 knockout $\left(\mathrm{Nrf}^{\circ /}\right)$ male mice (C57BL/6, $22 \mathrm{~g}$ to $27 \mathrm{~g}$ ) were obtained from the Breeding Colony of Roberto Bolli, University of Louisville. In order to ensure pure genetic lines, genotypes were examined by PCR analysis of genomic DNA isolated from tails. Mice were maintained on AIN76A chow (Purina) diet or pairedfed AIN-76 diet containing 0.45\% BHA (Purina Test Laboratories) for 7 days. Acrolein 
( $5 \mathrm{mg} / \mathrm{kg}$ body weight) was administered by gavage for 7 consecutive days while on AIN-76A diet. Control mice were either fed AIN76A diet or chow diet with water administration by gavage for the BHA and acrolein experiments, respectively. The mice were sacrificed $24 \mathrm{~h}$ after the last treatment and the livers were harvested and stored at $80^{\circ} \mathrm{C}$ until used. Procedures were performed in accordance with approval by UofL Institutional Animal Care and Use Committee (IACUC).

\section{RNA Isolation and Quantitative RT-PCR.}

RNA was isolated from mice liver or Hepa-1c1c7 using TRI reagent (Molecular Research Center, Inc., Cincinnati, OH) following standard protocol. Briefly, $\approx 0.1 \mathrm{~g}$ of frozen liver tissue was homogenized in $1 \mathrm{ml}$ of TRI reagent (Invitrogen, Carlsbad, CA). The RNA was purified using silica membrane spin columns (RNeasy reagent; Qiagen, Valencia, CA). The RNA integrity was assessed by running a $1 \%$ agarose gel and quantified using the Nano Drop spectrophotometer. The RNA was aliquoted and stored at $-80^{\circ} \mathrm{C}$ until used.

The mRNA levels of Aldh1a1, Aldh1a7, Aldh1b1, Aldh2, and the Nrf2 regulated genes (Ho-1, Nqo-1 and Gstm1) were assessed by qRT-PCR according manufacturer's instructions for absolute quantitation standard curve method. Briefly, total RNA isolated from mice liver or Hepa-1c1c7 were reverse transcribed to cDNA using the Advantage RT-for-PCR kit (Clontech, BD Bioscience, Palo Alto, CA) with random hexamer primers. RNase $\mathrm{H}$ was then used to degrade any residual RNA in the cDNA mix. QRTPCR was performed using the ABI 7900HT Sequence Detector System (Applied Biosystems, Foster City, CA) using gene specific FAM-labeled LUX primers (Invitrogen). The primer specificity was determined by BLAST analysis of predicted 
PCR products and the confirmation of a single PCR product by the melting curve analysis. A plot of the CT versus quantity of RNA was generated to verify linearity of amplification. All qRT-PCR experiments were performed in triplicate using cDNA sample from independent RNA sets and analyzed by the absolute quantitation standard curve method. All gene expression levels were normalized with 18S rRNA as endogenous control. QRT-PCR experiments were performed in triplicate using cDNA sample from independent RNA sets and data were expressed as the mean \pm SD and analyzed by Student's $t$ test. Values of $p<0.05$ were considered to be statistically significant.

\section{Cytosolic ALDH Activity}

The hepatic Aldh activity was examined in the cytosolic fractions isolated from the liver of male C57BL6 mice (Jackson Laboratories, Bar Harbor, MA) treated daily with acrolein ( $5 \mathrm{mg} / \mathrm{kg}$ by gavage) or sulforaphane ( $10 \mathrm{mg} / \mathrm{kg}$ by gavage) for 7 consecutive days or placed on BHA diet (0.45\% BHA in AIN76 diet) for 7 days and sacrificed $24 \mathrm{~h}$ after the last treatment. All procedures were performed following IACUC guidelines. The liver cytosolic fraction was isolated following protocol adapted in the Prough laboratory for preparation of liver microsomes (Remmer et al., 1966). Briefly, the livers were excised and cut into small pieces with scissors in a beaker of saline and homogenized in $0.25 \mathrm{M}$ sucrose buffered with $50 \mathrm{mM}$ potassium phosphate buffer $(\mathrm{pH}$ 7.4). The homogenized liver was then centrifuged at $1000 \mathrm{rpm}$ for $10 \mathrm{~min}$. Further sedimentation at $12000 \mathrm{rpm}$ for $20 \mathrm{~min}$ was then done in the same tube. The supernatant was then decanted leaving $5-10 \%$ of the supernatant with the pellet. The supernatant was sedimented at $108,000 \mathrm{~g}$ for $1 \mathrm{~h}$. To stabilize the enzymes, $20 \%$ glycerol was then added 
to the supernatant or cytosolic fractions and stored at $-80^{\circ} \mathrm{C}$. The Aldh activity was measured spectrophotometrically as described in Chapter II using $1 \mathrm{mM}$ propionaldehyde (substrate specific for Aldh1a1) as substrate.

\section{Western blotting}

Hepa-1c1c7 cells were treated with increasing doses of acrolein as described in the figure legends. Cells were harvested at different times and Western blot was essentially performed as described in the Method of Chapter II. HepG2 cells were also transfected with c-Jun or Nrf2 expression plasmids. Membranes were probed with antibodies against rat ALDH1 (1:2000) and GAPDH (1:10000) at room temperature for $2 \mathrm{~h}$ or c-Jun (1:1000), Jun D (1:1000), Nrf2 (1:1000) and phospho c-Jun (1:1000), JNK (1:1000) and phospho JNK $(1: 1000)$ at $4^{\circ} \mathrm{C}$ overnight. The membrane was incubated with horseradish peroxidase-conjugated goat anti-rabbit $(1: 10000)$ or goat anti-mouse secondary $(1: 10000)$ antibody and the transferred proteins were visualized with Amersham ECL Plus western blotting detection system.

\section{Transcription Factor Binding Sites Analysis}

The putative transcription factor binding sites of the murine Aldh1a1 5'-flanking region $(-8000 /+27 \mathrm{bp})$ was analyzed by Genomatrix MatInspector and Transfac software with emphasis on the binding sites for Nrf2 (A/GTGACNNNGC) and AP-1

(ATGAC/GTCA). Putative AREs and TRE were identified in the proximal promoter of Aldh1a1, suggesting that electrophile-mediated transcription of Aldh1a1 may be regulated by either $\mathrm{Nrf} 2$ or $\mathrm{AP}-1$. We cloned an $\approx 2.0 \mathrm{~kb}$ fragment of mouse Aldhlal proximal promoter that contains these putative ARE and TRE, upstream of pGL3 basic 
luciferase reporter vector using mouse genomic DNA (Promega, Madison, WI) as our template in PCR reaction.

\section{Plasmids and Cloning of Aldh1a1 Promoter Region}

Mouse genomic DNA was purchased from Promega (Madison, WI) and used in PCR experiment to generate 2,002 bp of mouse 5'-flanking sequence. This region contains $-1963 /+23$ bp relative to the transcription start site (TSS) of mouse Aldhla1 gene. The upstream primer (5'-GGTACCAAATGGGCAGGCATGGTAAC-3') was designed to introduce a Kpnl site while the downstream primer (5'AGATCTTGGTTTGGCTCCTGGAACAC-3') introduced a HindIII site. The PCR product was recovered into a pCR2.1 vector (Invitrogen, Carlsbad CA). The identity of the sequences of the PCR products was confirmed by sequence analysis in the University of Louisville, Center for Genetics and Molecular Medicine Nucleic Acid core facility. Kpn1 and HindIII enzymes were used to subclone the 2002 bp Aldh1a1 promoter region into the pGL3-Basic vector to generate -1963/+27Aldh1a1-Luc constructs.

The expression plasmid for c-fos (pRSV-cfos) and c-jun (pRSV-cjun) were provided by Pickett C.B and Nguyen T (Schering-Plough Research Institute, Kenilworth, New Jersey). The expression plasmid for Jun D (pcDNA3.1-JunD), Jun B (pcDNA3.1JunB), Fra-1 (pcDNA3.1-Fra1) and Fra2 (pcDNA3.1-Fra2) were kind gifts from Sunita K. Agarwal (NIDDK, NIH). The Nrf2 expression plasmid (pCI-Nrf2neo) was donated by K.S. Ramos (University of Louisville). The Plasmid 0.164GSTYa-ARELuc (0.164YaARELuc) containing 164 bases of the rat GST-Ya minimal promoter with a consensus ARE cloned upstream of the luciferase reporter gene was used as a positive control for Nrf2-dependent regulation of gene expression (Falkner et al., 1998). A construct 
containing the human collagenase TRE (pColTRELuc) was generated by Immaculate Amunom at the Prough laboratory. This plasmid was previously used as a positive control for AP-1 transcription activity.

\section{Transfection of HepG2 Cells and Luciferase Reporter Assays}

The human hepatoma cell line HepG2 (HB8065, American Type Culture Collection, Rockville, MD) was maintained in Eagle's minimal essential media with 10\% fetal bovine serum (HyClone, Logan UT), 1\% antibiotic-antimycotic solution (containing penicillin, streptomycin and fungizone; Invitrogen; Carlsbad CA) and non-essential amino acids (1X; Mediatech Inc. Herndon VA). Cells were plated at a density of approximately 150,000 cells $/ \mathrm{ml}$ per well in 12-well plate. After plating, cells were transfected using $1 \mu \mathrm{g} / 2.5 \mu \mathrm{L}$ of Lipofectamine LTX and $1 \mu \mathrm{L}$ of Plus (Invitrogen, Carlsbad, CA) with 250 ng/well of pGL0.5-1684Aldh1a1, pGL1.5-1684Aldh1a1, 1963/+27Aldh1a1, -1496/+27Aldh1a1-Luc, -1005/+27Aldh1a1-Luc, -480/+27Aldh1a1Luc, p0.164GSTYa-ARELuc or pColTRELuc. The cells were co-transfected with varying amounts of pRSV-cfos (0-80 ng), pRSV-cjun (0-80ng) or pCI-Nrf2neo (0-100 ng). After incubation at $37^{\circ} \mathrm{C}$ for $24 \mathrm{~h}$, the transfection media was replaced with $1 \mathrm{ml}$ media containing $10 \%$ FBS and then cells were treated when necessary with $0.1 \%$ DMSO or t-BHQ. The cells were harvested after overnight incubation $(\sim 17-24 \mathrm{~h})$ with $100 \mu \mathrm{L}$ of Luciferase cell lysis buffer (Promega, Madison, WI). The $\beta$-galactosidase and luciferase activities were determined as described by Falkner et al (1998). The data were expressed as luciferase activity relative to $\beta$-galactosidase activity to normalize for transfection efficiency. All transient transfection experiments were performed in triplicate and experiments were repeated at least twice for confirmation. Statistical comparisons among 
treatment groups were determined using a two-tailed t-test, with $\mathrm{p}<0.05$ as a criterion for significance.

\section{RESULTS}

\section{Induction of Aldh1a1 gene expression by acrolein and BHA in mouse liver}

Kinetic studies with recombinant Aldhla1 enzyme showed that reactive lipidderived aldehydes, such as acrolein and HNE are good substrates of Aldh1a1. These aldehydes are implicated in the pathogenesis of many oxidative stress-induced diseases. Thus mechanisms to induce the expression of Aldh1al might be beneficial against these pathologies. Previous studies demonstrate that the expression of Aldhla1 gene is modulated by a wide variety of endogenous and exogenous stimuli (Alnouti and Klaassen, 2008; Leonard et al., 2006). Microarray studies in the Prough laboratory indicated that acrolein feeding up-regulated Aldh1a1 $(\approx 2$-fold $)$ gene expression in mouse liver (Amunom, Makia and Prough, unpublished report). Hepatic Aldh1a1 mRNA levels were also induced $(\approx 3$-fold) by treatment of mice with other electrophiles, BHA. To confirm these microarray data, we examined whether acrolein or BHA can induce the expression of Aldhla1 gene in mice liver. C57BL/6 mice on laboratory chow diet were treated with water (control) or $5 \mathrm{mg} / \mathrm{kg}$ acrolein by gavage daily for 7 days. The daily human consumption of unsaturated aldehydes is estimated to be $\approx 5 \mathrm{mg} / \mathrm{kg}$ and no hepatocellular damage was observed in mice exposed to this dose of acrolein (Conklin et al., 2010), showing that $5 \mathrm{mg} / \mathrm{kg}$ is a sublethal concentration of acrolein. However, significant damage to the liver was observed when mice were administered an acrolein dose of $10 \mathrm{mg} / \mathrm{kg}$. Mice on AIN76 diet were also administered SFN (10 mg/kg) daily by gavage or fed AIN-76 diet containing $0.45 \%$ BHA for 7 days. Total RNA extracted from 
the liver was used for qRT-PCR analysis of gene expression of Aldhlal, Gstm I, Nqo-1 and $\mathrm{Ho}-1$ (Figure 7). Aldh1al mRNA levels were significantly increased in mice liver by acrolein $(\approx 3$-fold) or BHA (2.5-fold) treatment compared with control. The mRNA expressions of Aldh1a1 were also significantly increased when Hepa-1c1c7 cells were treated with $20 \mu \mathrm{M}$ acrolein for $6 \mathrm{~h}$ compared with control (Figure 8). As expected, acrolein treatment caused induction of antioxidant and electrophile detoxification genes, such as Gstm1, Nqo-1 and Ho-1 gene in mice liver. The mRNA expression of Gstm1, Nqo-1 and Ho-1 were also increased by BHA treatment in mice (Figure 7).

Even though, Ho-1 and Nqo-1 genes were significantly induced by SFN in mice liver, no induction of Aldh1a1 and Gstm1 gene expression was observed with SFN (Figure 7). These results suggest that the mechanism of induction of Ho-1 and Nqo-1 gene expression by electrophiles differs from that of Aldh1a1 or Gstm1. The isothiocyanate, SFN is a known activator of $\mathrm{Nrf2}$ (Nguyen et al., 2009), while BHA and acrolein are known to induce gene expression by activation of either Nrf2 or AP-1 (Choi and Moore, 1993;Lee and Murray, 2010). We conclude from Figures 7 and 8 that the signaling pathway involved in electrophile-mediated induction of Aldhla1 gene differs from that of Nqo1 and Ho-1. 


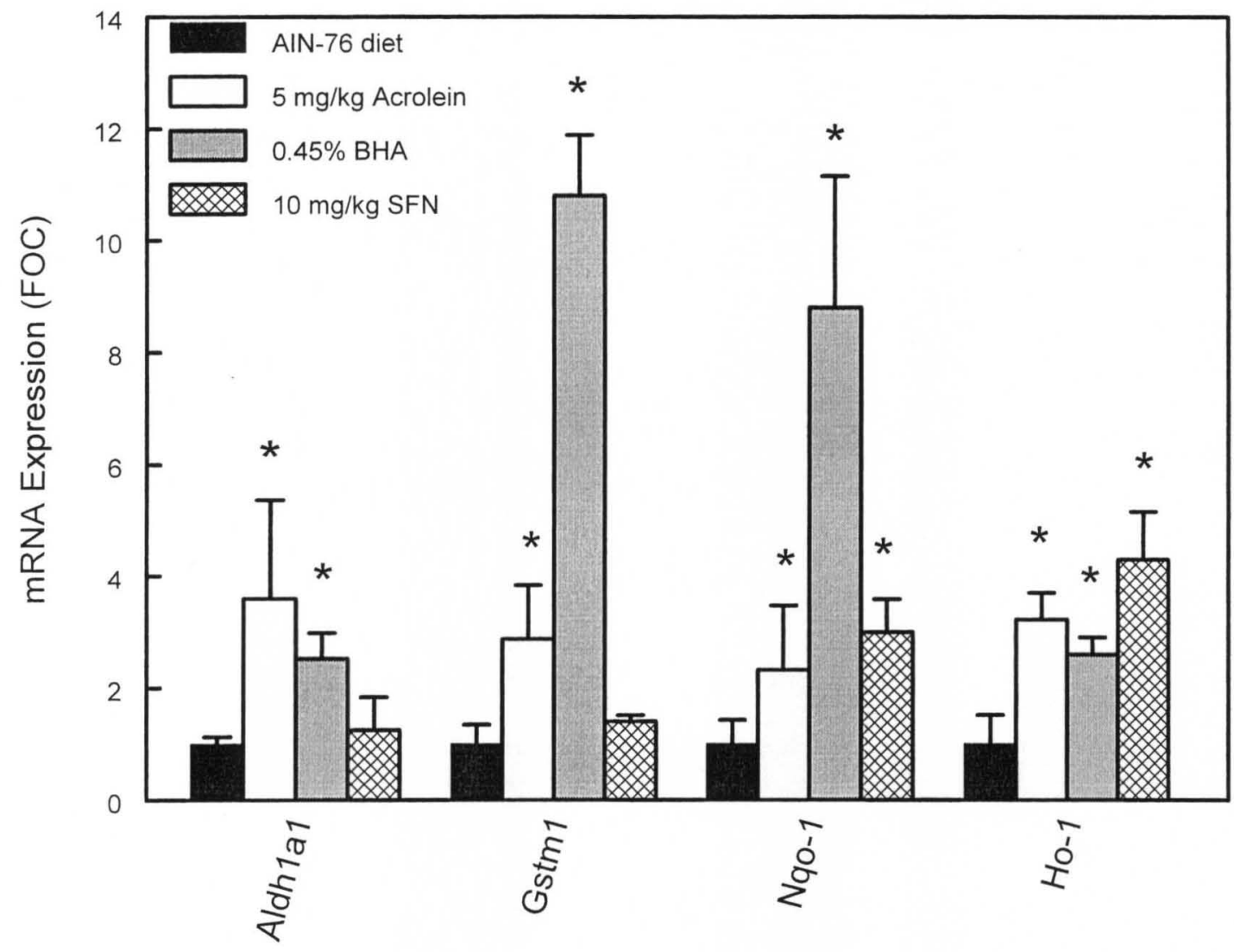

Figure 7. QRT-PCR analysis of electrophile detoxification gene expression in the liver of mice treated with acrolein, BHA or SFN. For the acrolein experiment, C57BL/6 mice on laboratory chow diet were treated with water (control) or $5 \mathrm{mg} / \mathrm{kg}$ acrolein by gavage daily for 7 days. For BHA and SFN experiment, C57BL/6 mice on AIN-76 diet were placed on diet containing $0.45 \%$ BHA for 7 days or administered $10 \mathrm{mg} / \mathrm{kg}$ SFN daily for 7 days by gavage $(n=4-5)$. Total RNA was extracted from the liver, reverse-transcribed to cDNA and qRT-PCR analysis of Aldhlal, Gstml, Nqo-1 and Ho-l gene expression was performed as described in Materials and Methods. ${ }^{*}$ is indicative of statistical significance compared to controls $(\mathrm{p}<0.05)$. 


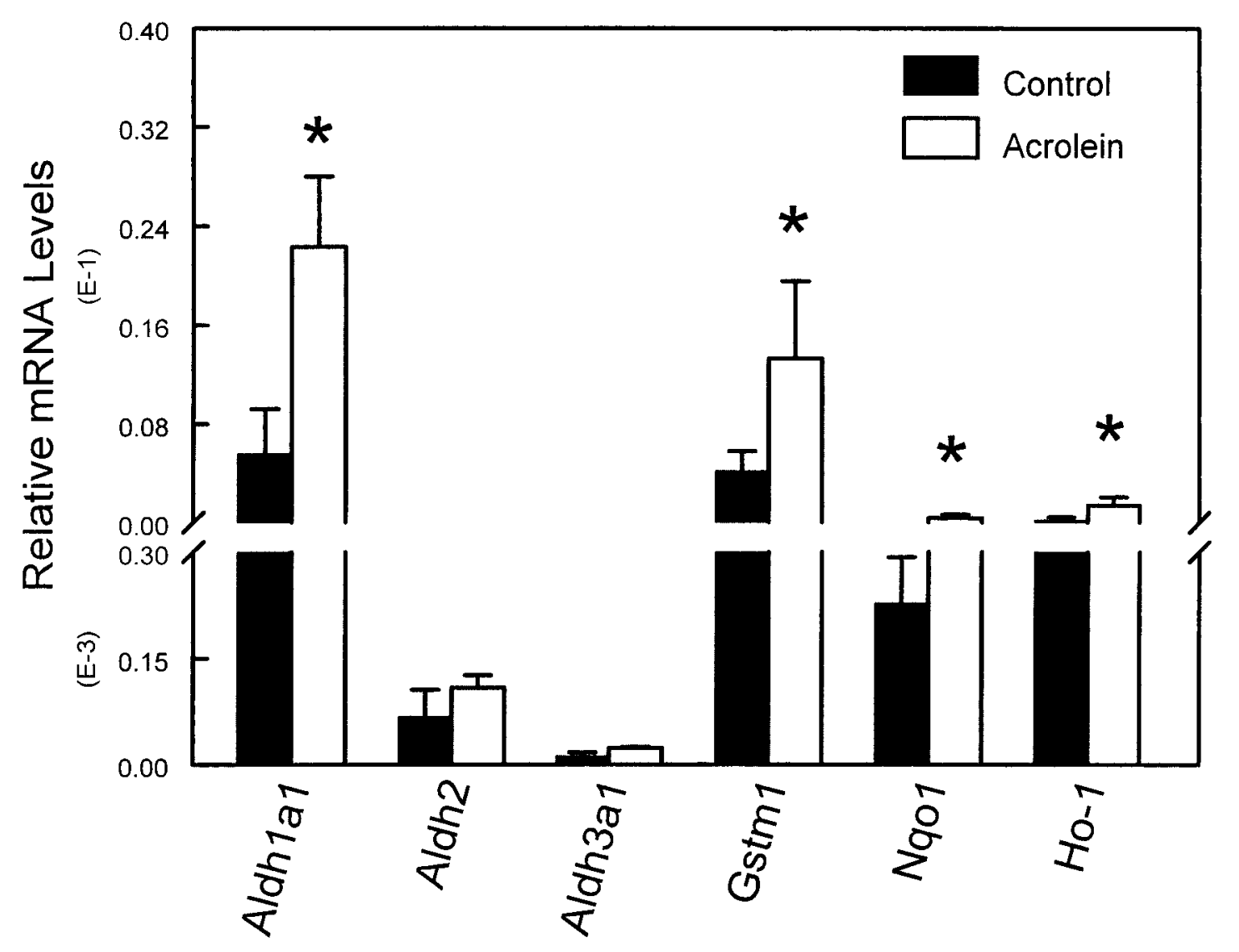

Figure 8. Acrolein induces the mRNA levels of cytoprotective genes encoding antioxidant and electrophile-detoxification enzymes in Hepa-1clc7 cells. Cells were untreated (control) or treated for $6 \mathrm{~h}$ with $20 \mu \mathrm{M}$ acrolein. Total RNA was extracted from the cells, reverse-transcribed to cDNA and gene expression in relation to $18 \mathrm{~S}$ was measured by qRT-PCR in triplicate using cDNA from independent sample sets. * indicates statistical difference compared with controls $(\mathrm{p}<0.05 ; 2$-tailed $\mathrm{t}$-test). 
Hepatic cytosolic Aldh activity was induced in mice treated with acrolein $(\approx 4-$ fold) or BHA ( $\approx 4.5$-fold) when $1 \mathrm{mM}$ propionaldehyde and $\mathrm{NAD}^{+}$was used as substrate and cofactor, respectively (Figure 9). However, the acrolein- or BHA-induced Aldh activity was nearly undetected when the assay was performed with $\mathrm{NADP}^{+}$as pyridine nucleotide cofactor. The major cytosolic Aldh isozymes are Aldh1a1 and Aldh3a1. From kinetic studies, we showed that propionaldehyde is a good substrate for Aldhlal and that Aldh1al utilized solely $\mathrm{NAD}^{+}$as cofactor. Moreover, since the mRNA levels of endogenous Aldh3al gene in normal mice liver is negligible, the major isoform that contributes to acrolein- or BHA-induced Aldh activity in mice liver is likely Aldhlal. This is the first report to demonstrate induction of Aldh gene expression by acrolein in mouse liver. Consistent with the mRNA expression, cytosolic Aldh activity was not induced in liver of mice by SFN even at a dose of $10 \mathrm{mg} / \mathrm{kg}$. These results validate our microarray data which demonstrate the induction of hepatic Aldhla1 gene expression and other electrophile detoxification gene by BHA or acrolein. We postulate from the SFN treatment results that Nrf2 may not be involved in electrophile-mediated induction of Aldhla1 gene. 


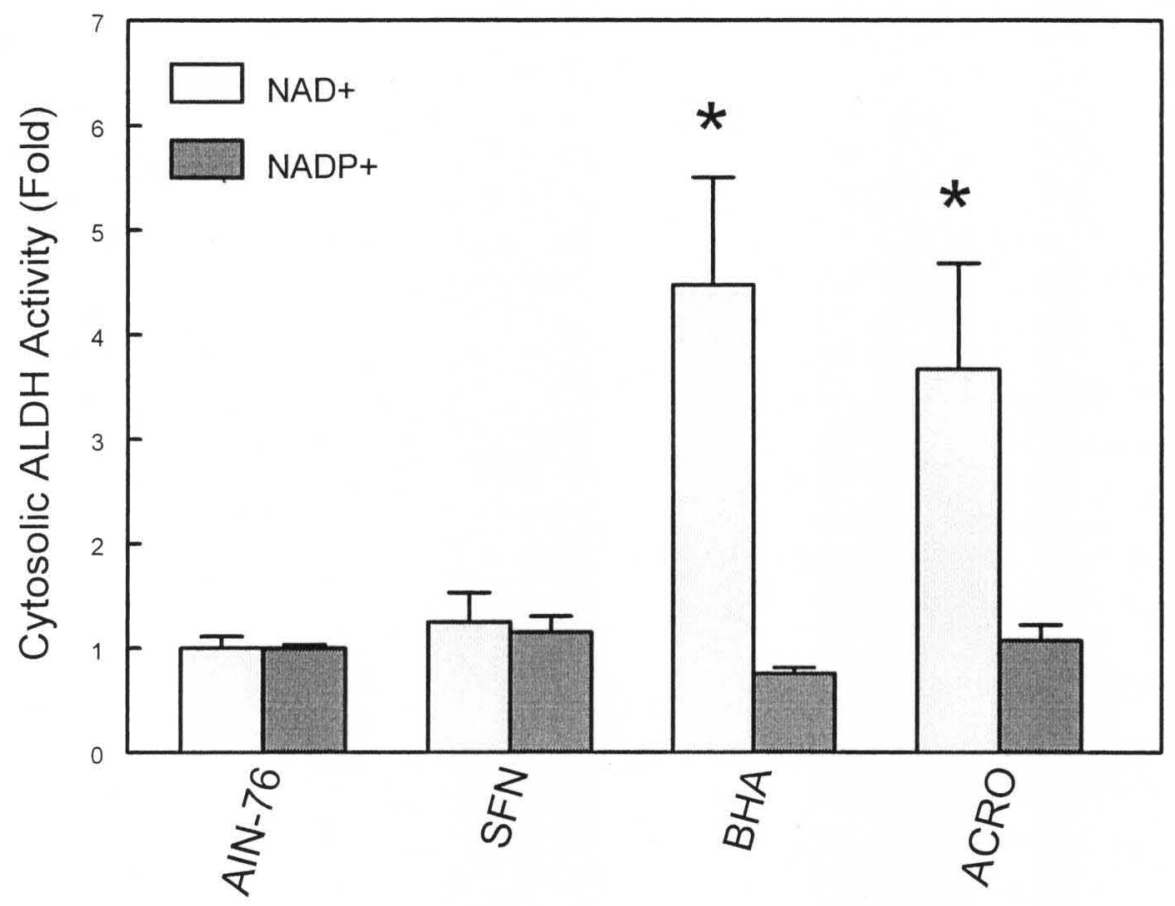

Figure 9. Increased liver cytosolic ALDH activity by BHA or acrolein in mice. C57BL/6 mice on normal chow diet were treated with water (control) or $5 \mathrm{mg} / \mathrm{kg}$ acrolein by gavage daily for 7 days. For BHA and SFN experiment, C57BL/6 mice on AIN-76 diet were placed on diet containing $0.45 \%$ BHA for 7 days or administered SFN $(10 \mathrm{mg} / \mathrm{kg})$ daily for 7 days by gavage $(n=4-5)$. The liver was extracted and the cytosol obtained by centrifugation at 108,000 $\mathrm{xg}$ for $1 \mathrm{~h}$. Aldh activity was assessed by monitoring the formation of $\mathrm{NAD}(\mathrm{P}) \mathrm{H}$ at $340 \mathrm{~nm}$ as previously described by Lindahl and coworkers (Lindahl and Petersen, 1991). The reactions rates were assayed spectrophotometrically at $37^{\circ} \mathrm{C}$ in a reaction mixture containing $1 \mathrm{mM}$ propionaldehyde with either $1 \mathrm{mM} \mathrm{NAD}^{+}$or $\mathrm{NADP}^{+}$as cofactor at $\mathrm{pH}$ 7.4. Specific activities (nmol/min $/ \mathrm{mg}$ of protein) for cytosols were $2.22 \pm 0.24\left(\mathrm{NAD}^{+}\right)$and $15.8 \pm 0.51\left(\mathrm{NADP}^{+}\right)$for controls; $9.97 \pm 2.28\left(\mathrm{NAD}^{+}\right)$and $11.95 \pm 0.88\left(\mathrm{NADP}^{+}\right)$for BHA-treated animals. * Significantly different from controls $(\mathrm{p}<0.05 ; 2$ tailed $\mathrm{t}$ test $)$. 


\section{Induction of Aldh1a1 gene expression by BHA in C57BL6 WT and Nrf2 ${ }^{-/-}$mouse liver}

The mechanism of BHA- or acrolein-induced transcription of Aldhlal is not completely understood. Previous studies have shown that the basal expression of Aldh1a1 was significantly reduced in $\mathrm{Nrf}^{-/-}$mice compared with WT. However, the mRNA levels of Aldh1a1 gene were not up-regulated in mice with liver-specific knockout of the negative regulator Keap-1, which showed constitutive activation of Nrf2. Our previous results also indicated that Aldh1a1 gene expression was not induced by SFN, a known activator of Nrf2 (Figure 7 and 9). However, the induction of gene expression by acrolein and $\mathrm{BHA}$ is dependent on activation of redox-sensitive transcription factors, such as $\mathrm{Nrf} 2$ and AP-1. Thus, in order to assess the role of Nrf2 in electrophile-induced transcription of Aldh1a1, C57BL/6 WT and Nrf2 ${ }^{-/}$mice were exposed to $0.45 \%$ BHA for 7 days. RNA was isolated from the mouse liver and the mRNA levels of Aldhlal and known Nrf2 target genes, such as Gstm1, Nqo1 and Ho-1, were examined by qRT-PCR.

The exposure of mice to BHA resulted in $\approx 2$-fold increase in mRNA levels of hepatic Aldh1al in both WT and $\mathrm{Nrf2}^{-/}$mice compared with untreated control of the same genotype (Table 2). However, the basal expression of Aldh1a1 was significantly reduced in $\mathrm{Nrf}^{-/}$mice compared with WT control mice of the same genotype. The upregulation of Nqo-1, Gstm1 and Ho-1 mRNA expression by BHA in WT mice was significantly reduced in $\mathrm{Nrf}^{-/-}$mice, suggesting that BHA-induced expression of these genes is dependent on Nrf2. However, the induction of Aldhlal gene by BHA in WT mice was not significantly different in $\mathrm{Nrf}^{-/-}$mice. These results indicate that BHAinduced expression of Aldhla1 is not mediated by Nrf2. 
Table 2

Effects of Nrf2 genotype on changes in mRNA levels of antioxidant and electrophile detoxification genes in response to dietary BHA

\begin{tabular}{|c|c|c|c|c|c|c|}
\hline \multirow{2}{*}{ Gene } & \multicolumn{2}{|c|}{ Constitutive Levels } & \multicolumn{2}{c|}{ Inducible Levels } & \multicolumn{2}{c|}{ Fold Induction } \\
\cline { 2 - 7 } & $\mathrm{Nrf2}^{+/+}$ & $\mathrm{Nrf2}^{-/}$ & $\mathrm{Nrf2}^{+/+}$ & $\mathrm{Nrf2}^{-/}$ & $\mathrm{Nrf2}^{+/+}$ & $\mathrm{Nrf2}^{-/}$ \\
\hline Aldh1a1 & 1.0 & 0.22 & 2.51 & 0.43 & $2.5^{*}$ & 2.0 \\
\hline Aldh1a7 & 1.0 & 0.38 & 2.07 & 0.66 & $2.1^{*}$ & 2.2 \\
\hline Aldh2 & 1.0 & 0.23 & 1.28 & 0.31 & 1.3 & 1.4 \\
\hline Aldh1b1 & 1.0 & 0.53 & 1.06 & 1.29 & 1.1 & $2.4^{* *}$ \\
\hline Gstm1 & 1.0 & 0.1 & 10.8 & 0.27 & $10.8^{*}$ & $2.6^{* *}$ \\
\hline Nqo1 & 1.0 & 0.11 & 8.8 & 0.48 & $8.8^{*}$ & $4.6^{* *}$ \\
\hline Ho-1 & 1.0 & 0.77 & 2.84 & 1.09 & $2.6^{*}$ & $1.4^{* *}$ \\
\hline
\end{tabular}

Values are the average fold change per genotype in the liver of WT and $\mathrm{Nrf}^{-/-}$mice. QRT-PCR analysis of gene expression in the liver of C57BL/6 $\mathrm{Nrf}^{+/+}$and $\mathrm{Nrf2}^{-/-}$mice in response to dietary administration of the phenolic antioxidant, BHA. Mice were fed either AIN-76A diet (control, $n=4$ ) or diet supplemented with $0.45 \%$ BHA (treated, $n=4$ ) for 7 days. The $\mathrm{Nrf2}^{-/-}$mice were fed chow diet only (control, $\mathrm{n}=3$ ) or diet supplemented with $0.45 \%$ BHA (treated, $n=3$ ). RNA extracted from the liver was reversed-transcribed to form cDNA. The mRNA levels of Aldh1a1, Aldh1a7, Aldh2, Aldh1b1, Ho-1, Nqo-1 and Gstm1 genes were analyzed by qRT-PCR. The fold induction is shown. For each point, a single mRNA was analyzed in triplicate and the average is shown. * indicates significantly different from WT control ( $\mathrm{p}<0.05 ; 2$-tailed t test). ** indicates significantly different from BHA-treated WT ( $\mathrm{p}<0.05 ; 2$-tailed t test). 


\section{Induction of AP-1 gene expression by acrolein or BHA in mouse liver}

In order to examine whether electrophile-induced expression of Aldhlal gene is dependent on AP-1, we assessed the mRNA levels of $c$-jun and $c$-fos in the liver of acrolein- and BHA-treated mice. We observed significant induction of $c$-jun and $c$-fos $(\approx 2.0$ fold $)$ in WT mice after BHA treatment (Figure 10A). This induction was significantly decreased in $\mathrm{Nrf}^{-/-}$mice, suggesting that c-Jun and c-fos genes expression are modulated by Nrf2. The expression of AP-1 genes in $\mathrm{WT}$ and $\mathrm{Nrf2} 2^{-/-}$mice correlates with that of Aldh1a1 gene (Figure 10A). Thus, the low basal expression of Aldh1a1 in $\mathrm{Nrf}^{-/-}$mice might be due to low levels of AP-1 genes in these mice. We also observed $\approx 4.0$-fold and 2.0 -fold induction in the mRNA levels of $c$-jun and $c$-fos genes respectively in the liver of mice after acrolein treatment (Figure 11A). The induction of mRNA levels of $c$-Jun and $c$-fos gene by acrolein observed in Hepa-1c1c7 cells was consistent with that observed in mouse liver (Figure 11A). We hypothesized that electrophile-induced expression of Aldh1a1 gene is mediated by AP-1 proteins. To further test our hypothesis, we assessed the protein levels and transcriptional activity of cJun in cellular extracts from WT mice exposed to BHA. Western blotting with antibodies against c-Jun and phospho-c-Jun was performed in extracts from the liver of WT mice exposed to control diet or diet containing BHA (Figure 10B). We observed significant increases in both protein levels and phosphorylation status of c-Jun in BHA-treated mice liver compare with control. To further test our hypothesis in Hepa-1c1c7, cells were exposed to increasing concentrations of acrolein for $4 \mathrm{~h}$ and the expression and activity of c-Jun were analyzed by immunoblotting (Figure 11B). Furthermore, Hepa-1c1c7 cells were treated with acrolein $(20 \mu \mathrm{M})$ at various time intervals and the activities of JNK and 

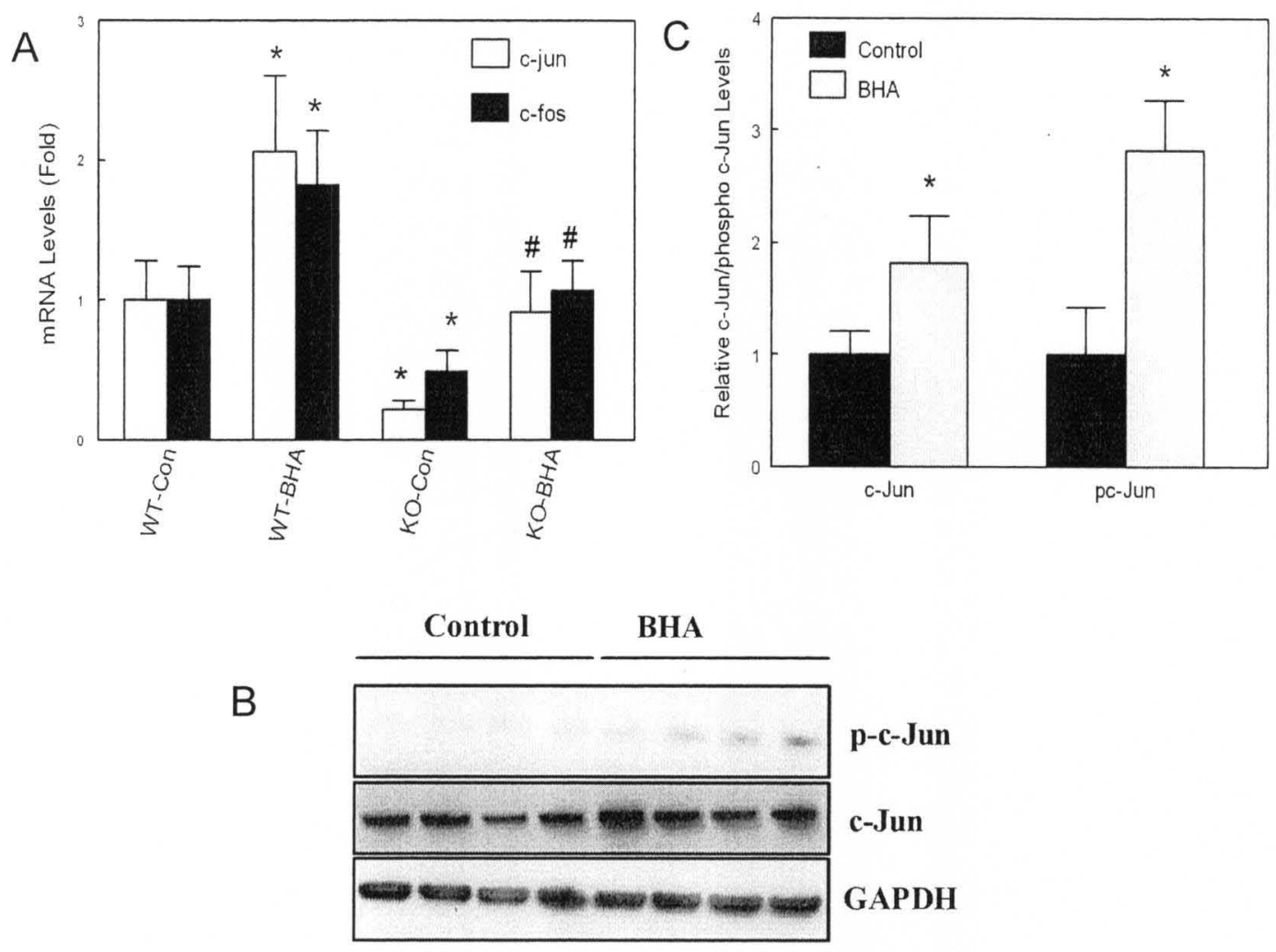

Figure 10. Effect of BHA on $c-j u n$ and $c$-fos gene expression in mouse liver. (A) $\mathrm{Nrf}^{+/+}$ or $\mathrm{Nrf2}^{-/}$mice on $\mathrm{C} 57 \mathrm{BL} / 6$ background were placed on $\mathrm{AIN}-76 \mathrm{~A}$ diet (control, $\mathrm{n}=4$ ) or diet supplemented with $0.45 \%$ BHA (treated, $\mathrm{n}=4$ ) for 7 days. QRT-PCR was used to examine mRNA levels of $c$-jun and $c$-fos. (B) C57BL/6 mice were fed either AIN-76A diet (control, $\mathrm{n}=4$ ) or diet supplemented with $0.45 \%$ BHA (treated, $\mathrm{n}=4$ ) for 7 days. Cell extracts were separated on SDS-PAGE gel and transferred onto nitrocellulose membranes. The membranes were incubated with antibodies against c-Jun (1:1000) and phospho-c-Jun (1:1000; Ser 63) overnight at $4^{\circ} \mathrm{C}$. (C) Densitometry analysis of c-Jun and c-Jun activation in response to BHA. Western blot was repeated three times and the figure is a representative blot. * and \# indicate statistical significant difference compared with $\mathrm{Nrf}^{+/+}$and $\mathrm{Nrf2}^{-/-}$controls, respectively $(\mathrm{p}<0.05 ; 2$-tailed $t$-test). 

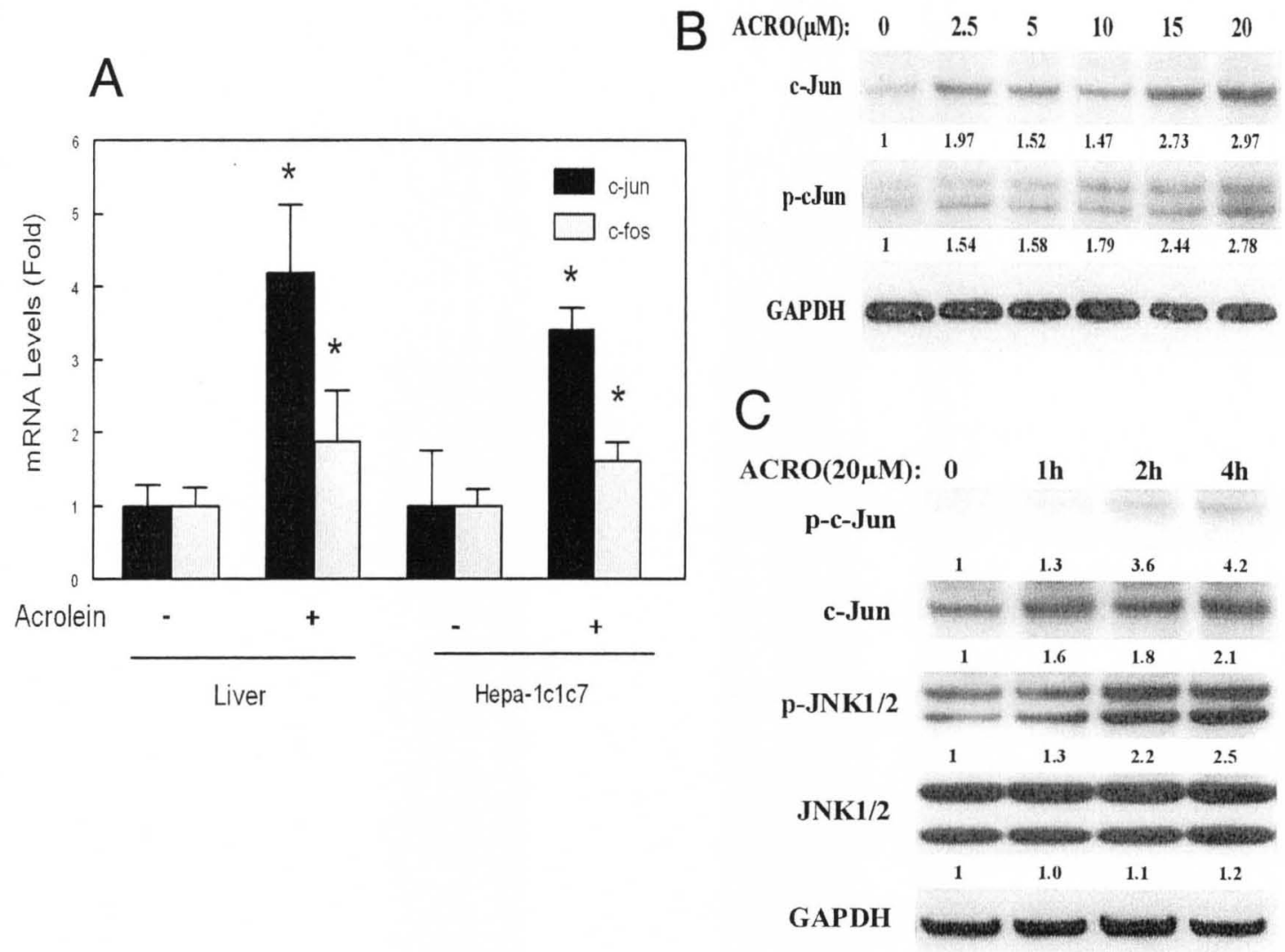

Figure 11. Effect of acrolein on $c-j u n$ and $c$-fos transcriptional activity in mouse liver and Hepa-1c1c7 cells. A. C57BL/6 mice were treated with water (control, $\mathrm{n}=5$ ) or $5 \mathrm{mg} / \mathrm{kg}$ acrolein (treated, $\mathrm{n}=5$ ) by gavage daily for 7 days. Hepa1c1c7 cells were exposed to 20 $\mu \mathrm{M}$ acrolein for $6 \mathrm{~h}$. The mRNA levels of c-jun and c-fos were assessed by qRT-PCR. B-C. Concentration- and time-dependent activation of c-Jun by acrolein. (B) Western blot of c-Jun protein level and activity. Hepa-1c1c7 cells were treated with varying doses of acrolein for $4 \mathrm{~h}$. (C) Cells were treated with $20 \mu \mathrm{M}$ acrolein at various time intervals. Membranes were incubated with antibodies against c-Jun phospho Ser63, c-Jun, JNK1/2 and p-JNK1/2. Western blot experiment was repeated three times and the figures shown are representative blot. Densitometry analysis is indicated by number below each blot. * Statistical difference compared to control ( $\mathrm{p}<0.05 ; 2$-tailed $\mathrm{t}$ test). 
c-Jun were analyzed by Western blot with antibodies against phospho-c-Jun and phospho-JNK (Figure 11C). Acrolein at a concentration of $2.5 \mu \mathrm{M}$ induced the protein levels and phosphorylation of c-Jun compared with control (Figure 11B). This induction was highest at an acrolein concentration of $20 \mu \mathrm{M}$. We also noticed increased phosphorylation of c-Jun, $2 \mathrm{~h}$ after treatment of cells with $20 \mu \mathrm{M}$ acrolein (Figure 11C). The kinetics of c-Jun phosphorylation is similar to that of JNK. Thus, the use of specific antibodies against phosphorylated $\mathrm{NK}$ implicates the $\mathrm{NK}$ signaling pathway in electrophile-induced expression of Aldh1a1 gene.

\section{Increased Aldh1a1 transcriptional activity by acrolein}

To further confirm that the mechanism of Aldh1a1 transcription by acrolein or BHA is mediated by AP-1, reporter gene assays using Aldh1a1 5'-flanking sequence (-1963 to +27 relative to the transcription start site) of Aldh1al gene was examined. This promoter region of Aldh1al gene contains putative AP-1 (-1516, -1069, -758 and -60$)$ and Nrf2 ($665,-1068$ and -1753 ) binding sites, whose sequence closely resembles the canonical TRE (TGACTCA) and ARE (TGACNNNGCA), respectively. We transfected HepG2 cells with Aldh1a1 luciferase construct (p2.0Aldh1a1) that contains the proximal promoter of Aldhlal gene cloned upstream of the luciferase gene. Twenty four hrs after transfection, cells were treated with $20 \mu \mathrm{M}$ acrolein for $6 \mathrm{~h}$ and luciferase activity was examined and normalized to $\beta$-galactosidase activity (Figure 12). As control for electrophile-induced transcription of ARE gene dependent on Nrf2, cells were transfected with pGSTYa-ARELuc construct that contains the ARE of the GST-Ya gene cloned upstream of a luciferase gene. We observed significant induction of Aldhlal promoter 
activity when cells were treated with acrolein $(\approx 1.8$ fold $)$ compared with untreated control (Figure 12). However, the induction seen with the luciferase assay was modest compared with the endogenous Aldh1a1 gene expression $(\approx 4$-fold $)$ in Hepa-1c1c7 cells observed by qRT-PCR (Figure 8). This might be due to the fact that acrolein treatment in the luciferase experiment was done in media containing low serum compared to serum free media in the qRT-PCR experiment. Furthermore, luciferase experiments were performed in HepG2 which might express higher endogenous levels of AP-1 compared to Hepa-1c1c7. HepG2 cells were chosen for the transient transfection and luciferase experiment due to high transfection efficiencies. However, as expected acrolein treatment resulted in $\approx 2.0$ fold induction in the luciferase activity of GSTYa-ARE (Figure 12). 


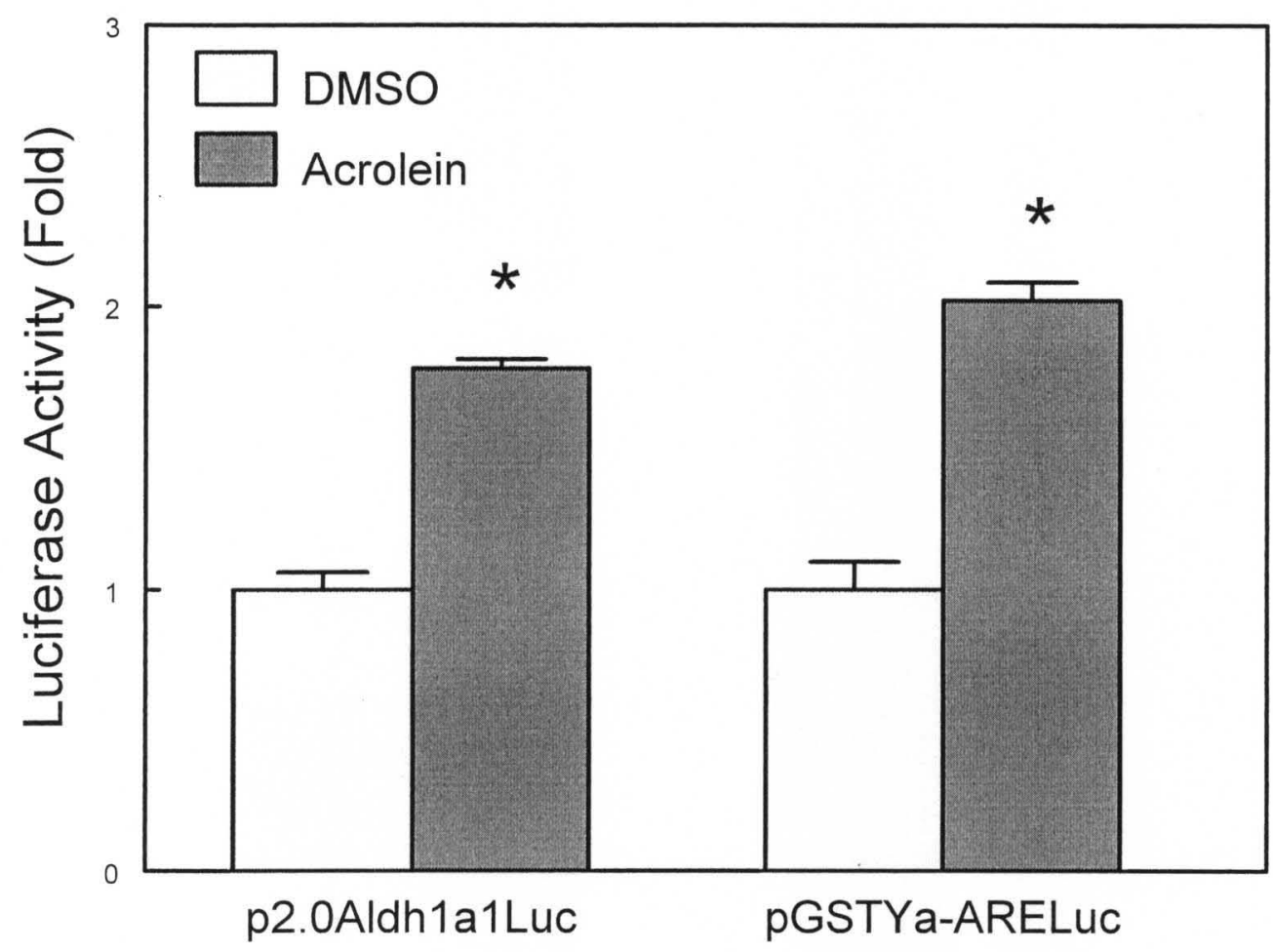

Figure 12. The Aldh1a1 transcriptional activity is significantly increased by acrolein. HepG2 cells were transfected with -1963/+27Aldh1a1 (p2.0Aldh1a1Luc) or GSTYaARE (positive control) luciferase plasmids for $24 \mathrm{~h}$. After transfection, cells were treated with either DMSO or $20 \mu \mathrm{M}$ acrolein for $6 \mathrm{~h}$ and luciferase activity was examined. Data are means $\pm \mathrm{SD}$ from at least three independent experiments. *Significant induction compared to vector transfected cells ( $\mathrm{p}<0.05 ; 2$ tailed $\mathrm{t}$ test). 


\section{Aldh1a1 transcriptional activity is not induced by Nrf2}

To examine whether the acrolein-mediated induction in Aldh1a1 promoter activity is dependent on Nrf2 transcription factor, we co-transfected cells with either p2.0Aldh1a1Luc or GSTYa-ARELuc constructs and increasing concentration of Nrf2 expression plasmids (Figure 14). We confirmed over-expression of c-Jun, JunD and Nrf2 in HepG2 cells by Western blot (Figure 13). We noticed a concentration-dependent increase in ARE-driven transcriptional activity with Nrf2 over-expression compared with vector control (Figure 14). However, the over-expression of Nrf2 failed to stimulate the promoter activity of Aldh1a1. The apparent lack of Nrf2-mediated Aldh1a1 transcriptional activity might be due to the absence of an electrophile or antioxidant responsive element in the cloned region of Aldh1a1 proximal promoter. Thus, we generated an Aldhlal luciferase construct containing $4.6 \mathrm{~kb}$ fragment of Aldhla1 promoter. However, there was still no significant difference in the Nrf2-mediated Aldh1al promoter luciferase activity compared to vector control when cells were transfected with p4.6Aldhlal construct (Figure 14). Thus, transient transfection experiments in HepG2 cells with Aldhlal 5'-flanking luciferase reporter constructs showed that co-transfection with Nrf2 expression plasmid alone or in the presence of $t$ BHQ had no effect (data not shown) of Aldh1a1 transcriptional activity. In addition to experiments with $\mathrm{Nrf}^{-/-}$mice and SFN treatment in WT mice, this result further confirmed that electrophile-induced expression of Aldh1a1 is independent of Nrf2. 


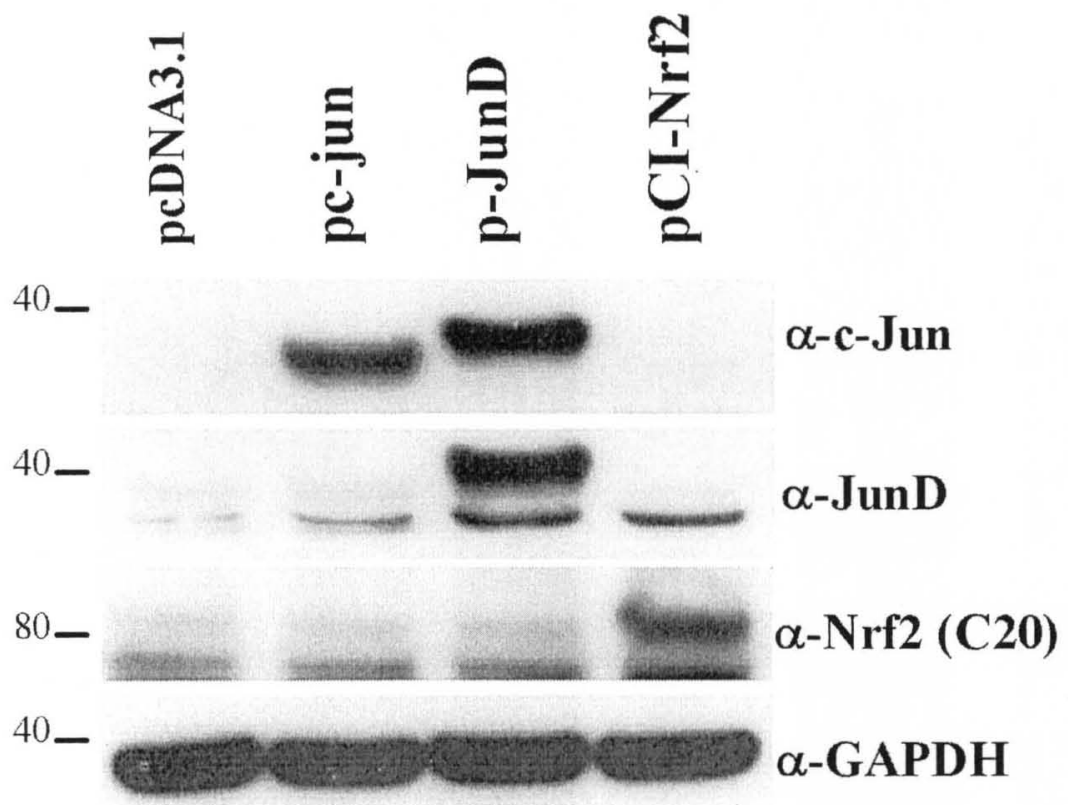

Figure 13. Western blots indicating over-expression of c-Jun, JunD and Nrf2 in HepG2 cells. HepG2 cells in 6 well plates were transfected with $2 \mu \mathrm{g}$ of pcDNA3, c-Jun, JunD or Nrf2 expression plasmid for $48 \mathrm{~h}$. After transfection, cell extracts were separated on SDS-PAGE gel and transferred onto nitrocellulose membranes. The membranes were probed with antibodies against c-Jun (1:1000; sc-45) and JunD (1:1000; sc-74) and Nrf2 $(1: 1000 ; \mathrm{C} 20, \mathrm{sc}-722)$. Western blot experiment was performed in triplicate and the figure is a representative blot. 


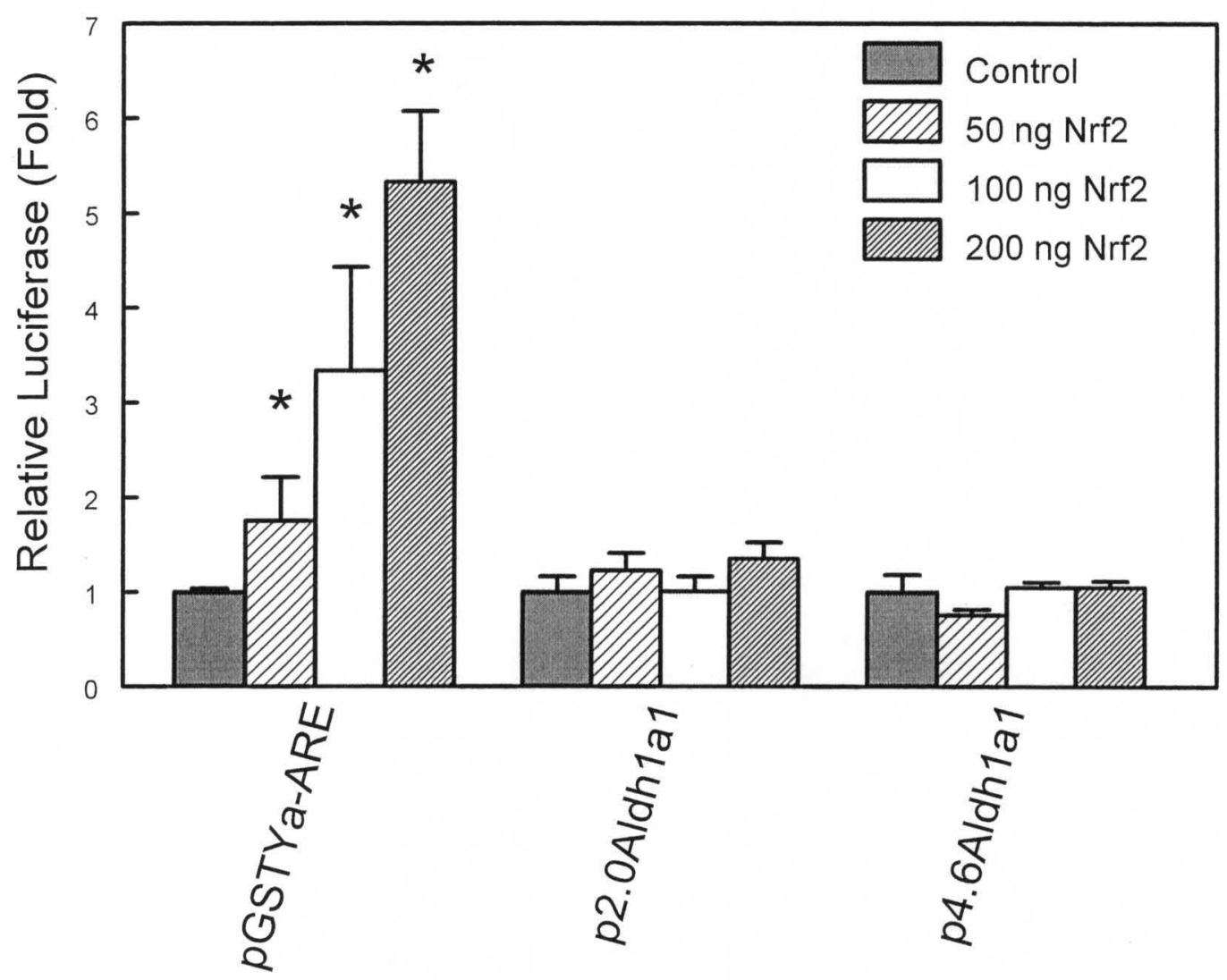

Figure 14. The Aldh1a1 promoter luciferase activity is not activated by Nrf2. HepG2 cells were transiently co-transfected with p0.164GSTYa-ARELuc (ARE control), p4.6Aldh1a1Luc or p2.0Aldh1a1-Luc luciferase construct and increasing concentrations of Nrf2 expression plasmid. Luciferase activity was normalized to $\beta$-galactosidase activity and corrected for the activities in cells that had been co-tranfected with empty plasmids. Data are means \pm SD from at least three independent experiments. * Significant induction compared to control vector-transfected cells ( $\mathrm{p}<0.05 ; 2$ tailed $\mathrm{t}$ test). 


\section{Increased Aldh1a1 transcriptional activity by AP-1}

We assessed the effect of AP-1 on Aldh1al transcriptional activity by transient transfection experiments in HepG2 cells with Aldh1a1 5'-flanking luciferase reporter construct. Cells were co-transfected with either p2.0Aldh1al or pColTRE and with c-Jun, c-fos or both c-Jun and c-fos expression plasmids. The pColTRE construct contains the TRE sequence from human collagenase gene upstream of a luciferase gene and has been used previously as a positive control for AP-1-dependent gene transcription. Overexpression of c-Jun resulted in $\approx 4$-fold induction in Aldh1a1 transcriptional activity (Figure 15). C-Jun over-expression was verified by Western blot (Figure 13). However, we noticed that the regulation of Aldh1a1 promoter activity by c-Jun and c-fos was different to that of the prototypic $\mathrm{AP}-1$ responsive gene, the human collagenase gene. As shown in Figure 15, both c-Jun and c-fos are required for induction of collagenase TRE luciferase activity. This synergism between c-Jun and c-fos for induction of collagenase TRE activity was absent in Aldh1al gene, suggesting that c-Jun/AP-1 might induce Aldh1al promoter activity as a homodimer and not as c-Jun/c-Fos heterodimer. To further test the hypothesis that electrophile-mediated transcription of Aldh1a1 is dependent on AP-1 and not the Nrf2 transcription factor, we assessed the role of c-Jun dominant negative protein (TAM67) on acrolein-mediated Aldhlal transcription (Figure 16). HepG2 cells were co-transfected with p2.0Aldh1a1 and with c-Jun, TAM67 or both c-Jun/TAM67 expression plasmids. Twenty four hrs after transfection, cells were treated for $6 \mathrm{~h}$ with $20 \mu \mathrm{M}$ acrolein. Acrolein-mediated increase in Aldh1al promoter activity was enhanced by over-expression of c-Jun. However, acrolein or c-Jun-induced 


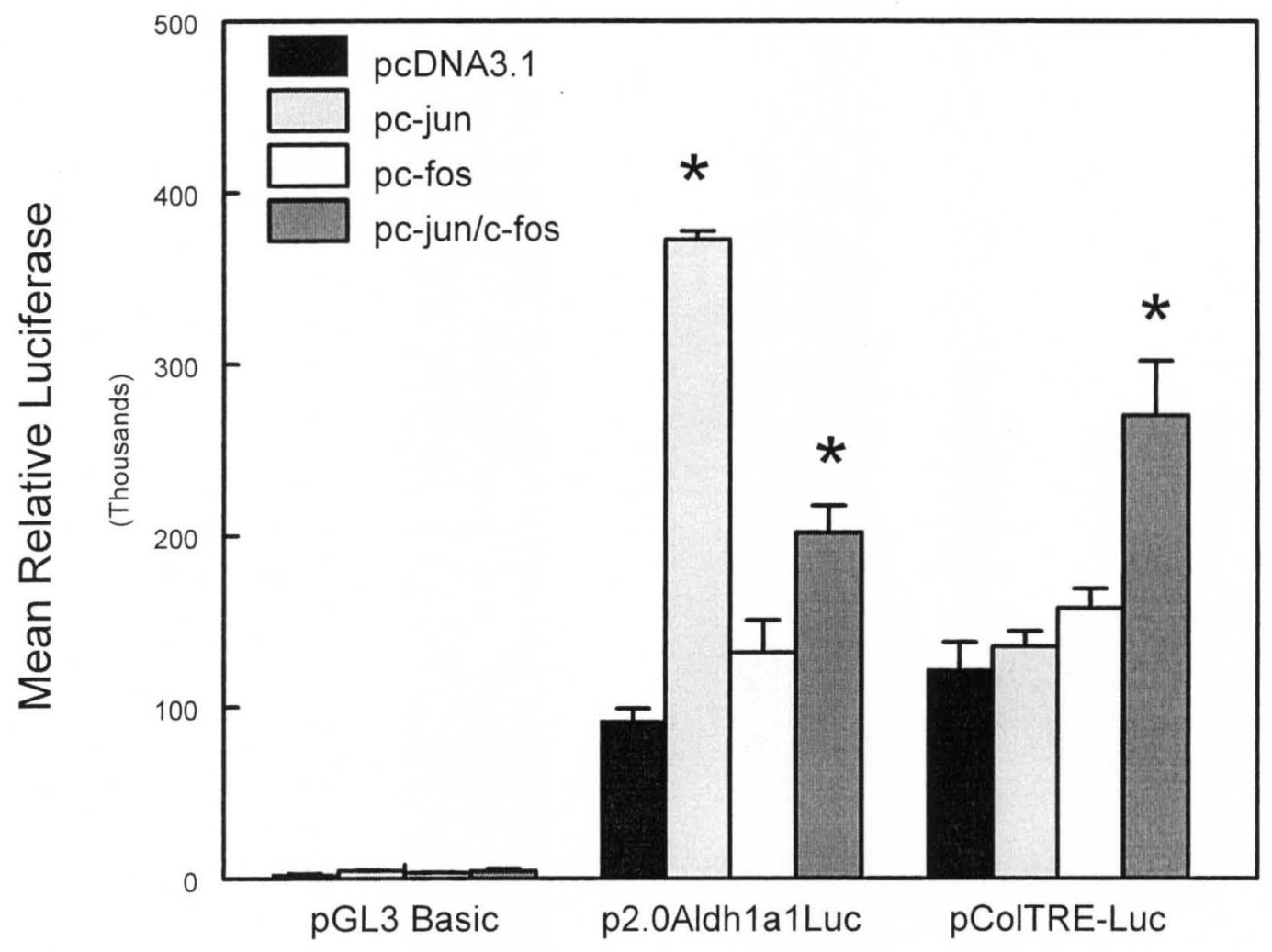

Figure 15. The Aldh1a1 promoter activity is transactivated by c-Jun and c-Fos. HepG2 cells were transiently co-transfected with -1963/+27Aldh1a1Luc (p2.0Aldh1a1Luc), pGL3 Basic or pColTRELuc luciferase construct with either c-Jun or c-Fos expression plasmid. Luciferase activity was normalized to $\beta$-galactosidase activity. Data are means \pm SD from at least three independent experiments. * Significant induction compared to vector transfected cells $(\mathrm{p}<0.05 ; 2$ tailed t test). 


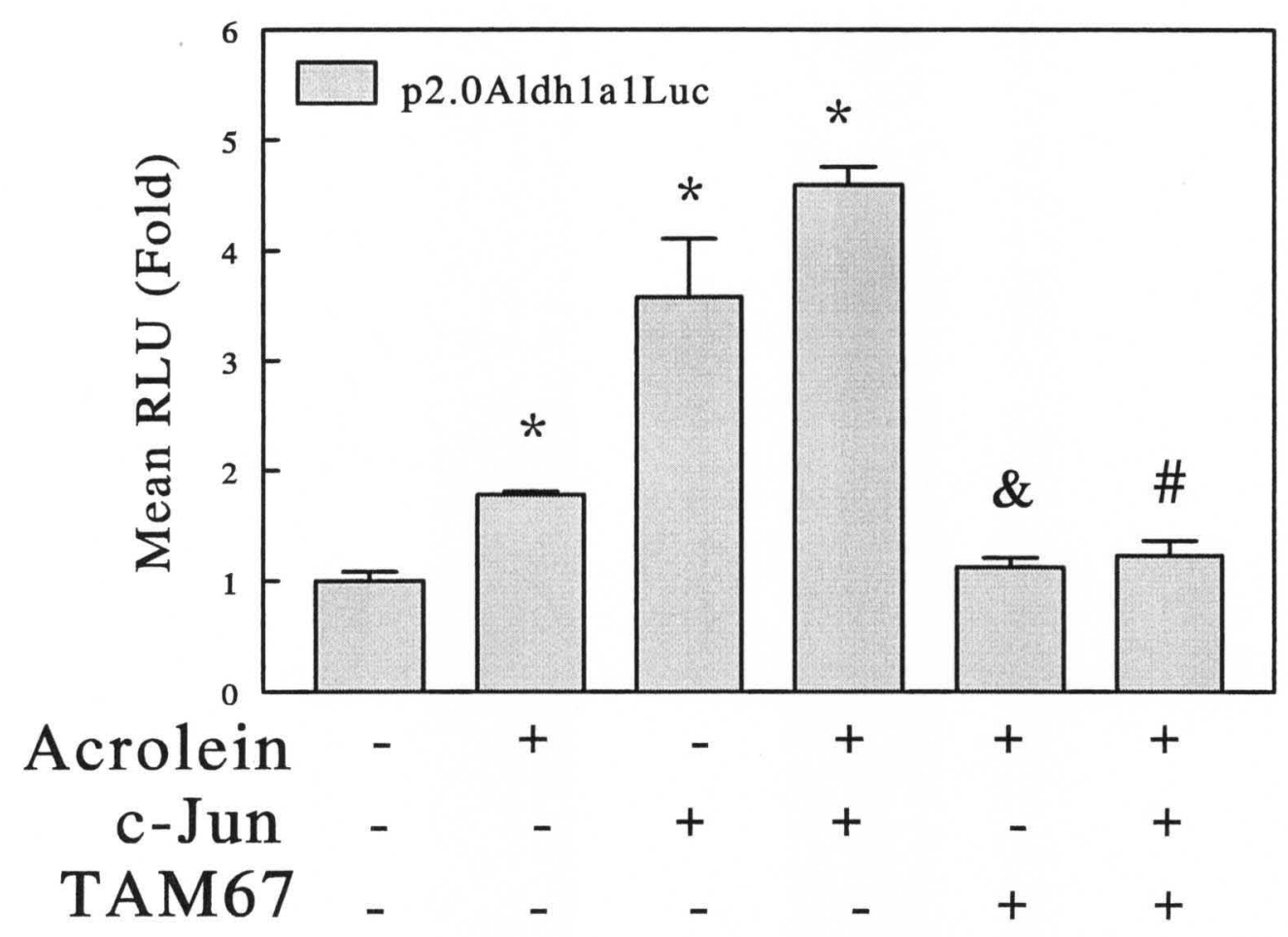

Figure 16. Inhibition of c-Jun mediated transactivation of Aldh1a1-luciferase activity by c-Jun dominant negative (TAM67) protein. HepG2 cells were transiently co-transfected with -1963/+27Aldh1a1Luc (p2.0Aldh1a1Luc) luciferase construct with c-Jun or TAM67 expression plasmid. $24 \mathrm{~h}$ after transfection, cells were treated for $6 \mathrm{~h}$ with DMSO or 20 $\mu \mathrm{M}$ acrolein. Luciferase activity was normalized to $\beta$-galactosidase activity. Data are means \pm SD from at least three independent experiments. *, significant difference compared to vector-transfected cells ( $\mathrm{p}<0.05 ; 2$ tailed $t$-test). \&, significant difference compared to acrolein-treated cells $(\mathrm{p}<0.05 ; 2$ tailed $\mathrm{t}$ test $)$ \# indicates significantly different from c-Jun transfected and acrolein treated cells. 
transcription of Aldh1a1 was abrogated by over-expression of TAM67, supporting the dependence on c-Jun/AP-1.

\section{The role of AP-1 family proteins on Aldh1a1 transcriptional activity}

We next examined the role of other AP-1 family members on Aldh1al gene transcription. Previous studies indicate that the jun family members (c-Jun, Jun D and Jun B) can induce the expression of genes as either homodimers or heterodimers with members of the fos (c-fos, Fos B, Fra-1 and Fra-2) family. We tested the effect of increasing concentrations of c-Jun, Jun D, Jun B or Fra-1 on Aldhlal transcriptional activity in HepG2 cells. As shown in Figure 17 , c-Jun $(\approx 3$ fold $)$, Jun D $(\approx 2.5$ fold $)$ or Jun $\mathrm{B}(\approx 4.5$ fold $)$ at $80 \mathrm{ng}$ concentration significantly transactivate Aldhlal promoter luciferase activity compared to control. However, at lower concentrations (20 ng), only cJun showed significant induction of Aldh1a1 luciferase activity, suggesting increased potency of the c-Jun transcription factor compared with the other Jun family members. We next transfected Hepa-1clc7 cells with either pcDNA3.1 (control) or jun family (cJun, JunD or JunB) expression plasmids to assess whether over-expression of these transcription factors can induce the protein levels of endogenous Aldhlal gene (Figure 18). Western blot analysis shows that over-expression of c-Jun, JunD or JunB in Hepa1clc7 cells increased Aldh1al protein compared to vector control transfected cells . These results further confirm that electrophile-mediated induction of Aldhlal is dependent on AP-1 genes.

We then assessed the role of the fos family (c-fos, Fra-1 and Fra-2) on Aldh1a1 transcriptional activity. The fos family are known to induce gene expression as 


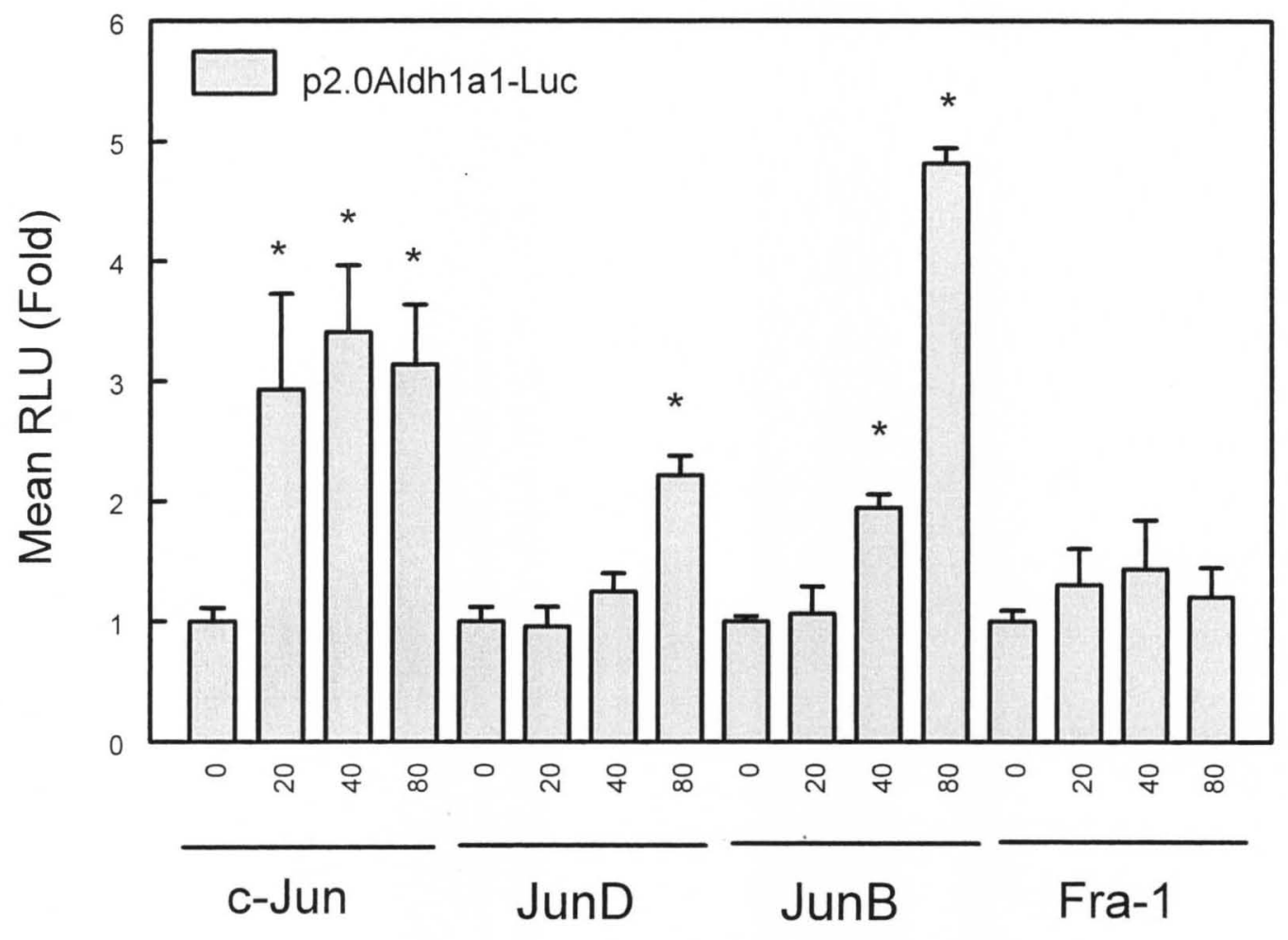

Figure 17. Effect of Jun family proteins on transactivation of Aldh1a1-luciferase activity. HepG2 cells were transiently co-transfected with -1963/+27Aldh1a1Luc (p2.0Aldh1a1Luc) luciferase construct with increasing concentration $(0,20,40$ and 80 ng) of c-Jun, Jun D, Jun B or Fra-1 expression plasmid. Luciferase activity was normalized to $\beta$-galactosidase activity. Data are means $\pm \mathrm{SD}$ from at least three independent experiments. ${ }^{*}$ Significant induction compared to vector transfected cells $(\mathrm{p}<0.05 ; 2$ tailed $\mathrm{t}$ test $)$. 


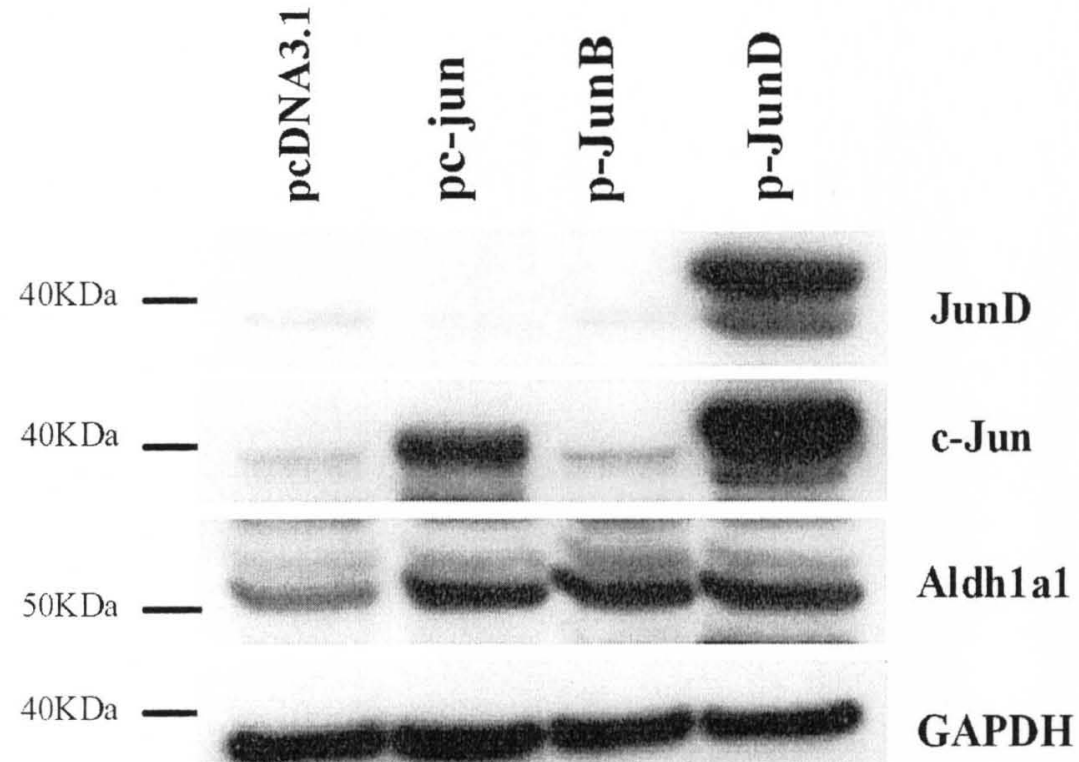

Figure 18. Over-expression of Jun family proteins in Hepa-1c1c7 increases the endogenous protein levels of Aldh1a1. Hepa-1c1c7 cells were transfected with $1 \mu \mathrm{g}$ of vector control (pcDNA3.1), c-Jun, JunB and JunD expression plasmid. $48 \mathrm{~h}$ after transfection, cell extracts were prepared with 1X RIPA buffer, separated on SDS-PAGE gel and transferred onto nitrocellulose membranes. The membranes were probed with antibodies against c-Jun (1:1000; sc-45) and Jun-D (1:1000; sc-74) and ALDH1 (1:1000). Western blot experiment was performed in triplicate and the figure is a representative blot. 


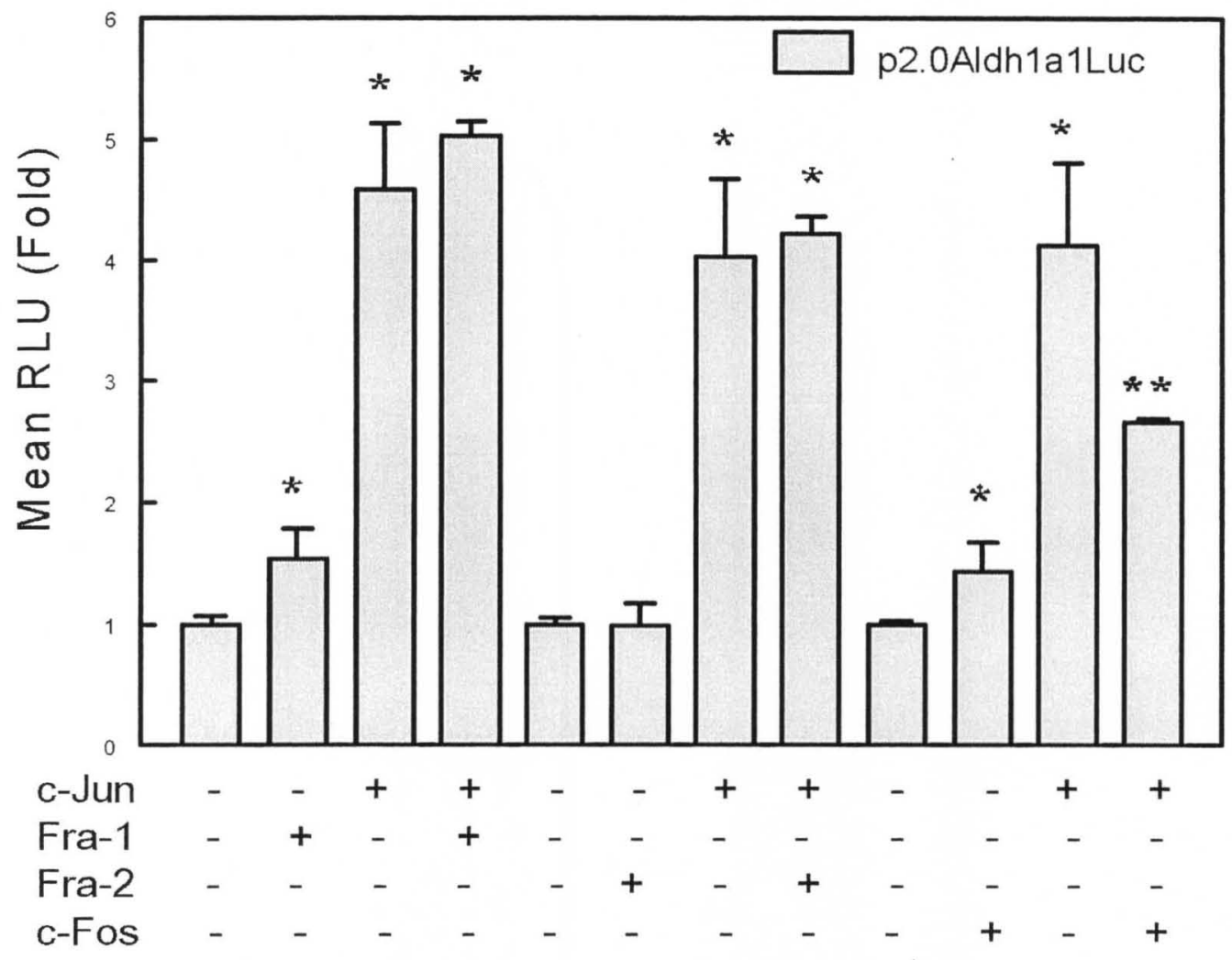

Figure 19. The effect of heterodimerization between c-jun and fos family proteins (c-fos, Fra-1 and Fra-2) on Aldh1a1 transcriptional activity. HepG2 cells were transiently cotransfected with -1963/+27Aldh1a1Luc (p2.0Aldh1a1Luc) luciferase construct with c-Jun and Fra1, Fra2 or c-fos expression plasmid. Luciferase activity was normalized to $\beta$ galactosidase activity. The results are presented as fold induction compared to vector control co-transfected cells. Data are means $\pm \mathrm{SD}$ from at least three independent experiments. * Significant induction compared to vector transfected cells $(\mathrm{p}<0.05 ; 2$ tailed $\mathrm{t}$ test). ${ }^{* *}$ Significantly different compared to $\mathrm{c}-J u n$ transfected cells $(\mathrm{p}<0.05 ; 2$ tailed $t$ test). 
heterodimer with Jun family. While over-expression of Fra-1 or Fra-2 had no effect on Aldh1al transcription either alone or with c-Jun, over-expression of c-fos inhibits c-Junmediated transactivation of Aldhla1 promoter activity (Figure 19).

To further test the effect of $\mathrm{c}-$ fos on Aldh1a1 promoter activity, we transiently cotransfected HepG2 cells with either p2.0Aldh1a1 or pColTRELuc and with constant amount of c-Jun or increasing concentrations of c-fos. While increasing concentrations of c-fos resulted in a concentration-dependent increase in the transcriptional activity of collagenase TRE, increasing concentrations of $\mathrm{c}$-fos caused a concentration-dependent decrease in Aldh1al promoter activity, which is suggestive of a dominant negative effect of c-Fos on c-Jun-mediated transactivation of Aldhlal promoter activity (Figure 20). These results indicated that transcriptional activation of Aldh1a1 promoter was mediated by c-Jun homodimer and not by c-Jun/c-Fos heterodimer. The preferred complex that binds to Aldh1a1 promoter during acrolein-dependent transactivation of Aldh1a1 gene appears to be c-Jun homodimer. Thus, over-expression of c-fos resulted in the formation c-Jun/c-fos heterodimer, thereby depleting the promoter of the active c-Jun homodimers. Previous studies showed that Nrf2 can induce the expression of cytoprotective genes by forming heterodimer with AP-1 proteins especially c-Jun (Lee and Murray, 2010). We examined whether there is cross-talk between c-Jun and Nrf2 in Aldh1a1 transcriptional activation. Cells were transfected with p2.0Aldh1a1 luciferase plasmids with varying concentrations of c-Jun and Nrf2 (Figure 21). Luciferase activity normalized to $\beta$-galactosidase activity was assessed in these cells $48 \mathrm{~h}$ after transfection. We observed that over-expression of Nrf2 had no effect on c-Jun-mediated transactivation of Aldh1al promoter luciferase activity (Figure 21). 


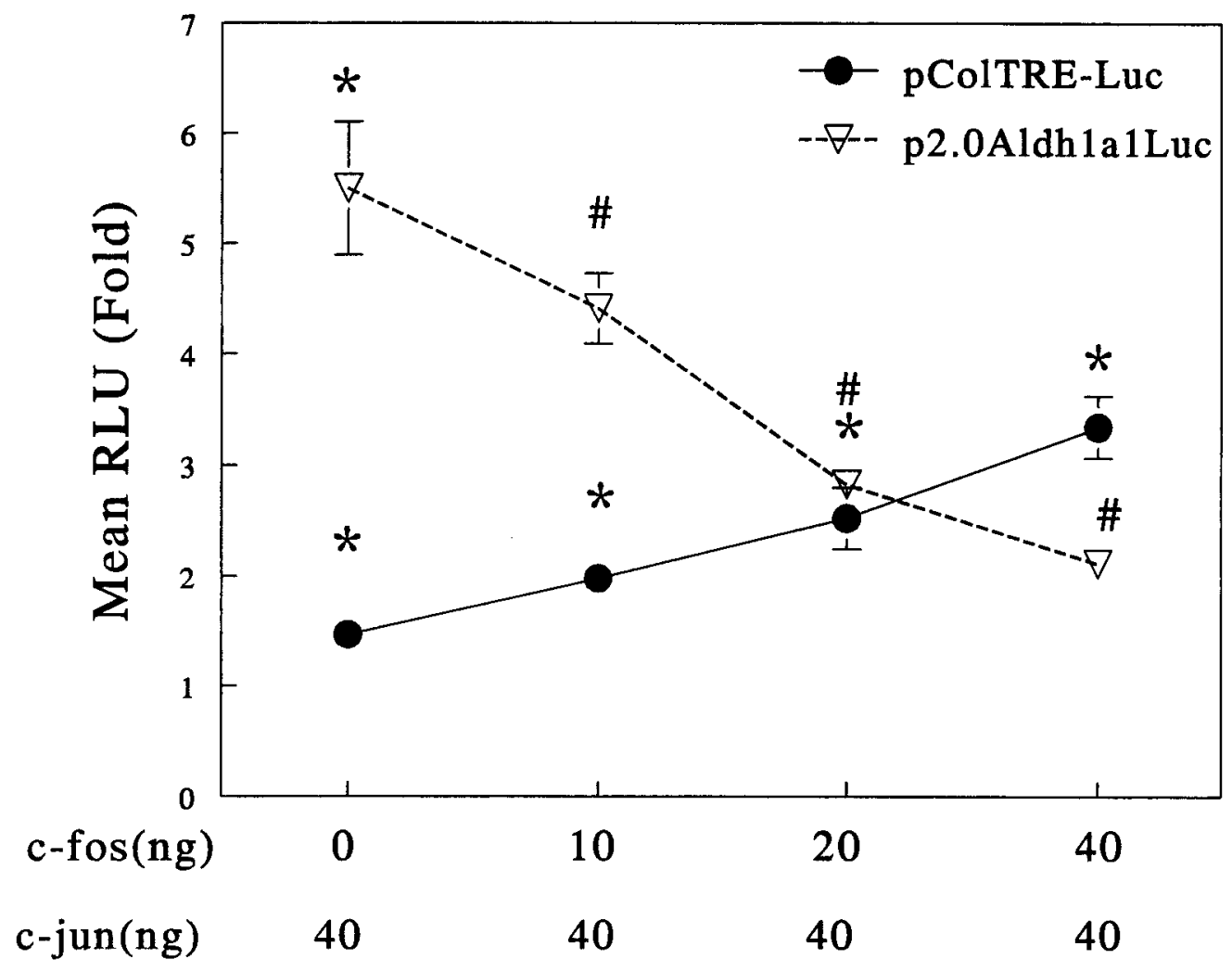

Figure 20. Induction of Aldh1a1 transcription is mediated by c-Jun homodimer and not by c-Jun/c-Fos heterodimer. HepG2 cells were transiently co-transfected with either pColTRE-Luc (TRE control) or -1963/+27Aldhla1 luciferase construct and increasing concentration of $c-j u n$ and $c$-fos expression plasmid. Luciferase activity was normalized to $\beta$-galactosidase activity. The results are presented as fold induction compared to vector control co-transfected cells. Data are means \pm SD from at least three independent experiments. * Significant induction compared to vector transfected cells $(\mathrm{p}<0.05 ; 2$ tailed $\mathrm{t}$ test). \# Significantly different from $\mathrm{c}$-Jun transfected cells $(\mathrm{p}<0.05 ; 2$ tailed $\mathrm{t}$ test). 


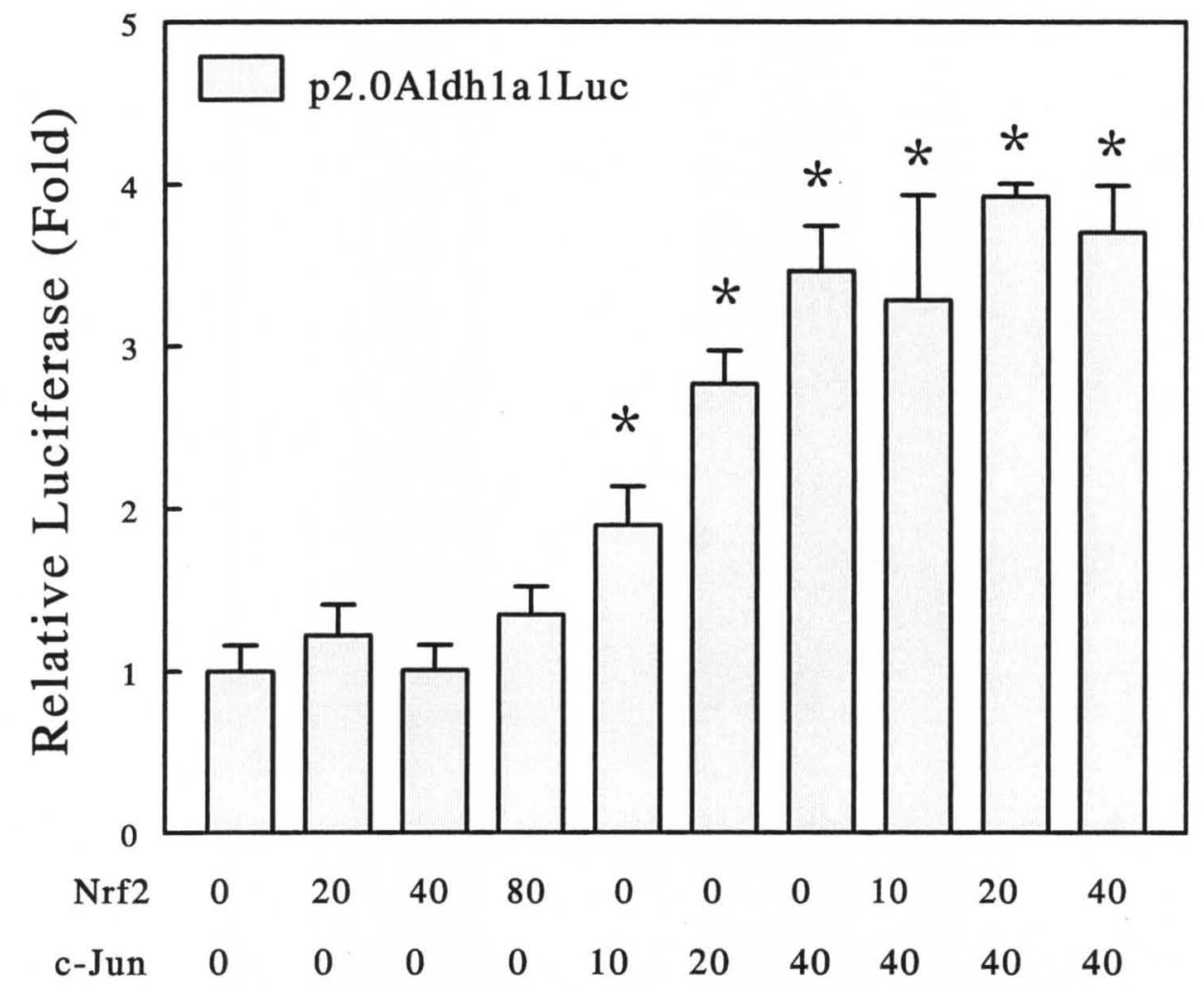

Figure 21. Lack of cross-talk between c-Jun and Nrf2 in transactivation of Aldh1a1 promoter activity. HepG2 cells were transiently co-transfected with -1963/+27Aldh1a1 luciferase construct with increasing concentrations of c-Jun and Nrf2 expression plasmid. Luciferase activity was normalized to $\beta$-galactosidase activity and corrected for the activities in cells co-tranfected with empty plasmids. Data are means \pm SD from at least three independent experiments. ${ }^{*}$ Significant induction compared to vector transfected cells ( $\mathrm{p}<0.05 ; 2$ tailed $\mathrm{t}$ test). 


\section{Aldh1a1 transcription is not induced by NF-кB activation in HepG2}

Previous studies indicate that electrophile-mediated transcription of cytoprotective genes is also dependent on NF- $\mathrm{BB}$ activation (Karin, 1999;Lamb et al., 2003; Yang et al., 2005). The NF-kB signaling pathway is highly sensitive to oxidative and electrophilic stress. Electrophiles can induce the phosphorylation and degradation of $\mathrm{I} \kappa \mathrm{B}$, resulting in nuclear accumulation of NF- $\kappa \mathrm{B}$. Moreover, the proximal promoter of Aldh1al ( -1963 to +27 relative to transcription start site) contains putative NF- $\mathrm{BB}$ binding sites at positions -638 and -1139 relative to the transcription start site. We assessed the effect of activation of NF- $\kappa$ B by TNF $\alpha$ on Aldh1al transcriptional activity.

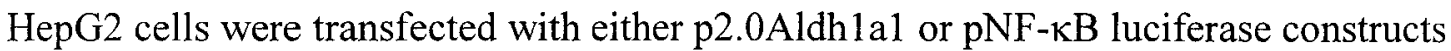
for $24 \mathrm{~h}$. After transfection, cells were treated with either $0.5 \mathrm{ng} / \mu \mathrm{l}$ or $1.0 \mathrm{ng} / \mu \mathrm{l} \mathrm{TNF} \alpha$ for $4 \mathrm{~h}$ (Figure 22a). As expected, we observed a concentration-dependent increase in NF- $\mathrm{kB}$ responsive luciferase activity by TNF $\alpha$. However, Aldh1al promoter activity was not responsive to TNF $\alpha$ even at a concentration $(1 \mathrm{ng} / \mu \mathrm{l})$ known to activate $\mathrm{NF}-\kappa \mathrm{B}$. This indicates that the regulation of Aldh1a1 transcription by electrophiles is not directly

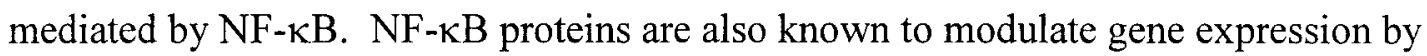
forming active heterodimers with AP-1 proteins (Rahmani et al., 2001). We further assessed whether there is cross-talk between the NF- $\mathrm{kB}$ signaling pathway and c-Jun/AP1 on regulation of Aldhlal gene transcription. Cells were co-transfected with Aldh1al luciferase plasmid and c-Jun expression plasmids. Cells were then treated with TNF $\alpha$ (1 $\mathrm{ng} / \mu \mathrm{l})$ for $4 \mathrm{~h}$. The c-Jun mediated induction of Aldhla1 promoter activity was significantly reduced when cells were treated with TNF $\alpha$. These results suggest that NF$\kappa \mathrm{B}$ had little or no direct effect on 

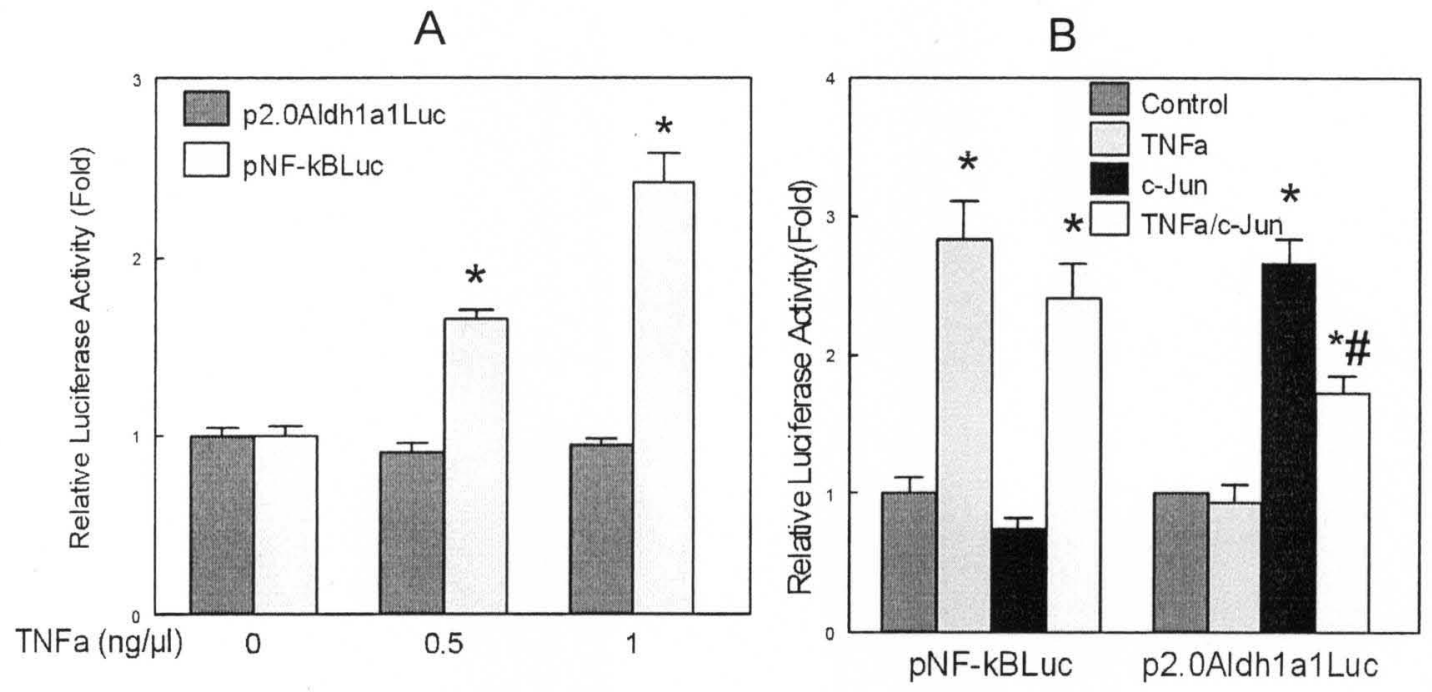

Figure 22. Aldhlal promoter activity is not responsive to activation of NF- $\kappa$ B signaling pathway by TNF $\alpha$. HepG2 cells were transfected with either $250 \mathrm{ng}$ p2.0Aldh1a1Luc or

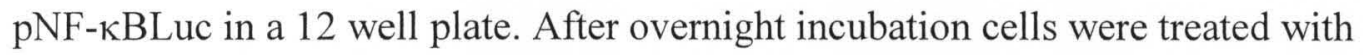
increasing concentrations of TNF $\alpha$ as indicated for $4 \mathrm{~h}$ (A) or with $1 \mathrm{ng} / \mu \mathrm{l} \mathrm{TNF} \alpha$ for $4 \mathrm{~h}$ (B). Data are means \pm SD from at least three independent experiments. * Significant induction compared to vector transfected cells ( $\mathrm{p}<0.05 ; 2$ tailed $\mathrm{t}$ test). \# indicates significantly different from $c$-jun transfected cells ( $p<0.05 ; 2$ tailed t test). 
Aldh1a1 promoter activity, but it apparently alters AP-1-dependent Aldh1a1 transcriptional activity in a yet to be defined manner.

\section{Role of MAPKs in electrophile-mediated transcription of Aldh1a1}

The activities of AP-1 proteins are regulated by MAPK, such as p38, ERK1/2 and JNK. The activation of JNK by oxidative stress or electrophile is known to phosphorylate its downstream target c-Jun, resulting in increased transcriptional activity of c-Jun. We examined the role of MAPK in electrophile-mediated transcription of Aldhlal gene. HepG2 cells were co-transfected with -1963/+27Aldh1a1 luciferase construct and a c-jun expression plasmid. Cells were pre-treated with $0.4 \%$ DMSO, $25 \mu \mathrm{M}$ SP600125 (JNK inhibitor), $50 \mu \mathrm{M}$ PD98059 (MEK inhibitor) or $40 \mathrm{nM}$ OA (protein phosphatase 2a inhibitor) for $17 \mathrm{~h}$. Cells were then treated with either $0.4 \% \mathrm{DMSO}$ or $20 \mu \mathrm{M}$ for $6 \mathrm{~h}$ (Figure 23). Acrolein-mediated increases in Aldhla1 transcriptional activity were diminished when cells were pretreated with JNK (SP600125) or MEK (PD98059) inhibitors. The inhibition of the JNK signaling pathway also abrogated the c-Junmediated transactivation of Aldh1a1 promoter activity. However, pretreatment of cells with the phosphatase $2 \mathrm{~A}$ inhibitor, OA had a small but insignificant effect on acrolein- or c-Jun-mediated transcriptional activation of Aldh1a1 gene. These results indicate that JNK signaling pathway play a critical role in electrophile-mediated transcriptional activation of Aldhlal gene. 


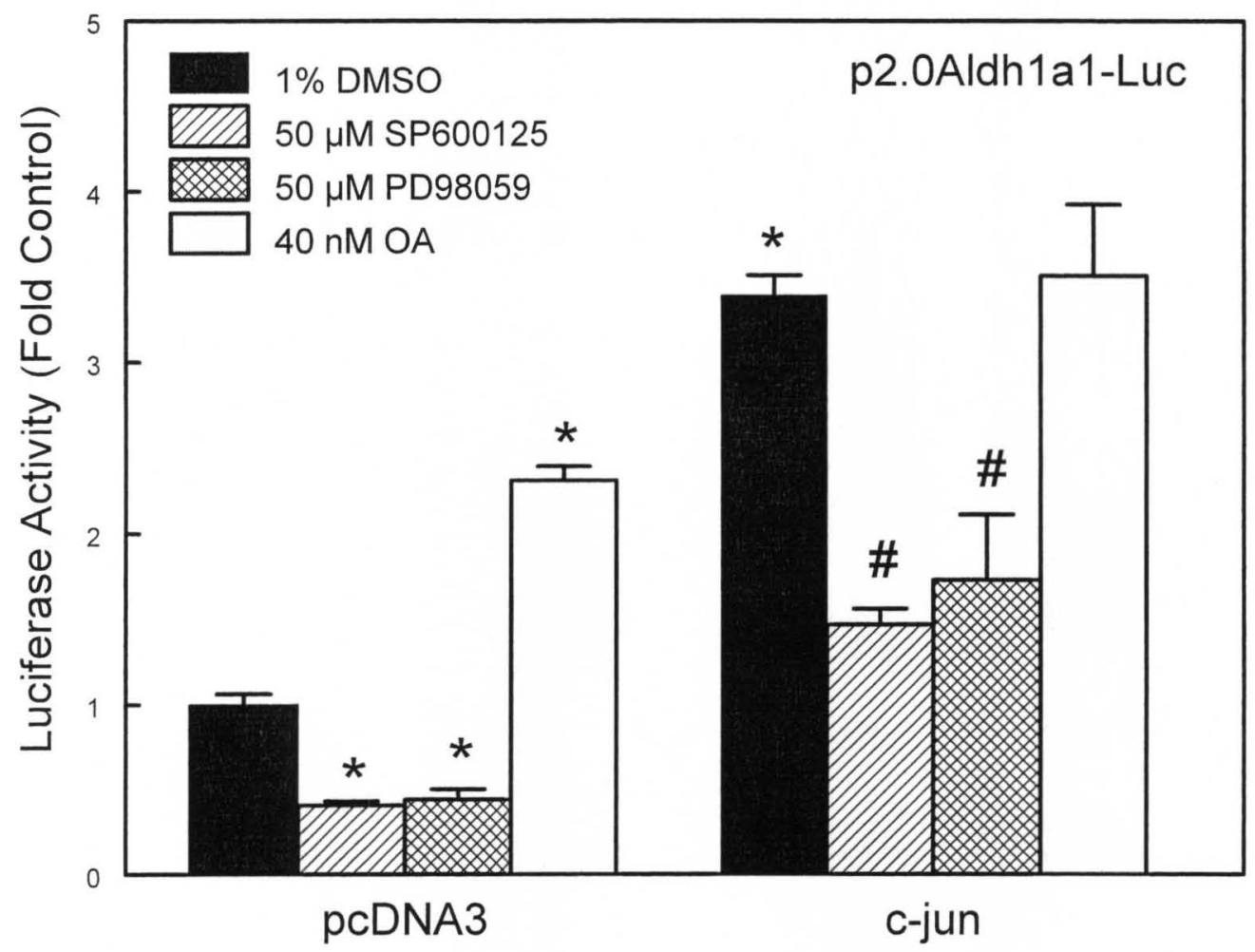

Figure 23. The role of MAPKs in electrophile-mediated transactivation of Aldh1a1 promoter activity. Aldh1a1 transcriptional activity is significantly reduced by inhibition of JNK (SP600125) and MEK (PD98059) kinase and increased by inhibition of protein phosphatase 2A (Okadaic acid) activity in HepG2 cells. HepG2 cells were co-transfected with p2.0Aldh1a1 (-1963/+27Aldh1a1) luciferase construct and a c-jun expression plasmid. Cells were treated with $0.4 \%$ DMSO, $50 \mu \mathrm{M}$ SP600125, $50 \mu \mathrm{M}$ PD98059 or 40 $\mathrm{nM}$ OA for $17 \mathrm{~h}$. Data are means \pm SD from at least three independent experiments. * Significant induction compared to vector transfected cells $(\mathrm{p}<0.05 ; 2$ tailed $\mathrm{t}$ test). \# indicates significantly different from $c$-jun transfected cells ( $\mathrm{p}<0.05 ; 2$ tailed $\mathrm{t}$ test). 


\section{DISCUSSION}

\section{The regulation of Aldh1a1 gene expression by BHA and acrolein}

Reactive oxygen species are readily generated in the liver during normal metabolism of foreign compounds and fatty acids, and even higher levels are produced in pathological conditions, such as steatosis. The ROS-mediated destruction of membrane lipids and free fatty acids results in the formation of lipid aldehydes by a chain AND autocatalytic reaction known as lipid peroxidation. The reactive lipid-derived aldehydes are most likely responsible for the pathophysiological effects of ROS and are implicated in the pathogenesis of several oxidative stress-associated pathologies, such as steatohepatitis, liver fibrosis, cancer and aging. Cells have developed elaborate systems to cope with these toxic electrophiles, thereby preventing aldehyde-induced oxidative damage. Our previous study to evaluate the sensitivity of cells with low expression of Aldh1a1 indicate that lipid aldehydes, such as acrolein and HNE are readily detoxified by Aldh1a1 (Makia et al., 2011). Moreover, high levels of Aldhlal gene expression in the liver and lens, two organs noted for generation of ROS and cytotoxic lipid aldehydes suggest the importance of Aldh1a1 in cellular protection against oxidative stress-induced damage. Thus, mechanisms to induce the expression of Aldhlal gene in mouse liver may be a useful strategy to prevent oxidative stress-induced diseases, such as the progression of hepatic steatosis to steatohepatitis.

Previous studies indicate that Aldhlal gene expression is induced by a variety of microsomal enzyme inducers, such as phenobarbital (PB) and BHA (Alnouti and Klaassen, 2008). Moreover, the expression of several genes encoding antioxidant and electrophile detoxification enzymes, including Aldh1a1 was specifically elevated in 
mouse liver by electrophiles such as BHA and acrolein (Amunom, Makia and Prough, unpublished report). The induction of Aldh1a1 and other electrophile detoxification genes, such as Nqo-1, Ho-1 and Gstm1 in mice liver by acrolein or BHA was confirmed by qRT-PCR (Figure 7). Consistent with the mRNA levels, the cytosolic Aldh activity was significantly induced in BHA or acrolein treated-mice liver, when $1 \mathrm{mM}$ propionaldehyde and $\mathrm{NAD}^{+}$was used as substrate and cofactor, respectively (Figure 9), to measure Aldhlal-specific enzyme activation. Enzyme kinetic studies with recombinant mouse Aldhs (Table 1) indicate that propionaldehyde is a good substrate for Aldh1al and that Aldh1a1 solely uses $\mathrm{NAD}^{+}$as cofactor. Furthermore, the expression of cytosolic Aldh3a1 is negligible, while Aldhlal is highly expressed in normal mice liver, suggesting that the predominant cytosolic isoform of Aldh induced in mice liver by BHA or acrolein is Aldh1a1. Thus, cytosolic Aldh1a1 activity is induced in mice liver by acrolein or BHA compared to control mice.

\section{b. The role of Nrf2 in electrophile-mediated activation of Aldh1a1 gene expression}

The signaling pathway involved in electrophile-mediated transactivation of Aldhlal gene in mice liver is not clearly understood. However, BHA and acrolein are known to stimulate the expression of cytoprotective genes by direct activation of redoxresponsive transcription factors or by indirect activation of protein kinase signaling pathway, such as MAPK. To assess the role of Nrf2 in electrophile-mediated transcription of Aldhlal gene, we fed C57BL6 WT or $\mathrm{Nrf2}^{-/}$mice diet containing BHA for 7 days and analyzed the mRNA expression of Aldh1al and Nrf2-target genes, Nqo1, Ho-1 and Gstml by qRT-PCR (Table 2). BHA-induced expression of Nqo1, Ho-1 and

Gstm1 genes in WT mice were significantly reduced in $\mathrm{Nrf}^{-/}$mice, implicating Nrf2 in 
BHA-induced expression of these genes. However, the fold-induction of Aldh1a1 mRNA expression by BHA in WT mice was not significantly different from $\mathrm{Nrf}^{-/-}$mice, indicating that BHA-mediated transcription of Aldh1a1 gene is not dependent on Nrf2. Furthermore, we demonstrated that the prototypic Nrf2 activator, SFN did not induce Aldh1al gene expression in mouse liver (Figures 7 and 9). These results were consistent with previous studies using keap1-knockdown (keap1-kd) mice with constitutively active Nrf2 in the liver (Reisman et al., 2009). The mRNA levels of Nqo1 and Gstm1 genes were significantly increased in keap1-kd mice compared to WT control, suggesting that over-expression of $\mathrm{Nrf} 2$ transcriptionally activates these genes. However, Aldh1a1 mRNA expression in the livers of keapl-kd mice was comparable to that of WT mice, supporting the hypothesis that the induction of Aldh1al gene expression by electrophiles is regulated by Nrf2-independent mechanism.

The molecular mechanism underlying transcriptional regulation of Aldhla1 gene by electrophiles was further examined by analysis of the $8.0 \mathrm{~kb}$ region of the $5^{\prime}$-flanking sequence of the mouse Aldhlal gene for AP-1-like (TRE) and Nrf2-like (ARE) binding sites using the Genomatrix MatInspector Professional consensus sequence identification program. The region between -1963 to $+27 \mathrm{bp}$ relative to the transcription start site containing AP-1-like (-1516, -1069, -758 and -60) and Nrf2-like (-665, -1068 and -1753) binding sites was cloned upstream of the luciferase reporter plasmid. Transient transfection experiments in HepG2 demonstrate that Aldh1a1-luciferase reporter containing these $c i s$-regulatory elements is responsive to acrolein (Figure 11). Acrolein induced an approximately 1.8-fold increase in Aldh1al-luciferase activity, demonstrating that -1963 to +27 region of Aldh1al promoter is responsive to electrophiles. 
Electrophiles promote nuclear accumulation of $\mathrm{Nrf} 2$ by causing its dissociation from keap1. Nrf2 then binds to an ARE in the promoter of target gene resulting in increased gene expression (Nguyen et al., 2004;Nguyen et al., 2009;Rushmore et al., 1991). While there was a concentration-dependent activation of GSTYa-ARE-luciferase activity by Nrf2, increasing concentrations of $\mathrm{Nrf} 2$ failed to stimulate the activity of Aldh1a1luciferase reporter (Figure 14). A possible explanation for the lack of responsiveness to Nrf2 of Aldh1a1-luciferase reporter could be due to the absence of a functional ARE in the region $(-1963$ to $+27 \mathrm{bp})$ of Aldhla1 promoter used for our experiments. In addition, Aldh1a1-luciferase construct containing $4.6 \mathrm{bp}$ region of Aldh1a1 promoter was also unresponsive to Nrf2, which further lends support to our hypothesis that electrophileinduced expression of Aldhla1 gene is independent of Nrf2.

\section{The role of AP-1 in BHA or acrolein-mediated activation of Aldh1al gene}

We hypothesized that electrophile-induced transcription of Aldhlal gene is mediated by activation of AP-1 transcription factor. The induction of antioxidant and electrophile detoxification genes by electrophiles is known to be mediated by stimulation of the expression and activity of AP-1 genes. For example, oxidative stress-induced expression of human ferritin $\mathrm{H}$ gene was dependent on activation of JunD (Tsuji, 2005). Expression $c$-jun and $c$-fos genes were significantly increased by BHA or acrolein in the liver of WT mice (Figure 10 and 11). While the mRNA expression of c-fos in the liver of WT mice by BHA or acrolein compared to control were similar $(\approx 2$-fold $)$, we observed a 4-fold increase in c-jun mRNA expression by acrolein and a 2-fold increase by BHA. The mRNA levels of c-jun and c-fos gene were also significantly increased by acrolein in 
Hepa-1c1c7 cells compared to untreated cells. The basal expression of c-jun or c-fos genes were significantly reduced in $\mathrm{Nrf}^{-/-}$mice compared to WT control. Moreover, the induction of c-jun and c-fos gene by BHA in WT mice was reduced in $\mathrm{Nrf}^{-/ \cdot}$ mice, suggesting that the Nrf2 modulates the basal and BHA-induced expression of the AP-1 genes. Previous studies in $\mathrm{Nrf}^{-/-}$mouse embryonic fibroblast (MEF) also reported the regulation of AP-1 gene expressions by Nrf2 (Yang et al., 2005). Thus, the low basal expression of c-Jun and c-fos possibly explained the reduced expression of Aldh1a1 gene observed in the liver of $\mathrm{Nrf2}^{-/}$mice not treated with BHA. The phenolic electrophilic compounds, such as BHA have been reported to augment the protein expression and activity of AP-1 genes (Choi and Moore, 1993;Li et al., 2005;Tsuji, 2005; Yang et al., 2002). The transactivation potential of c-Jun is enhanced by N-terminal phosphorylation through JNK (Eferl and Wagner, 2003). WT mice treated with BHA showed increased protein expression and phosphorylation of c-Jun compared to control (Figure 10). We also observed increased phosphorylation of c-Jun, $2 \mathrm{~h}$ after treatment of Hepalclc 7 cells with acrolein $(20 \mu \mathrm{M})$ (Figure 11). The phosphorylation of c-Jun, $2 \mathrm{~h}$ after treatment correlates with the activation of its upstream kinase JNK. These results suggest that electrophile-induced transcription of Aldh1al gene is mediated by enhanced expression and activity of AP-1 genes.

Co-transfection of HepG2 cells with collagenase TRE-luciferase reporter constructs and c-Jun, c-fos or c-Jun/c-fos expression plasmids demonstrate that both cJun and c-Fos were required to activate the collagenase TRE-luciferase activity (Figure 15 and 20). However, c-Jun strongly activated (4-fold) Aldh1al-luciferase reporter, while co-transfection with plasmids encoding c-Fos abrogated reporter activity, 
suggesting that c-Jun activates Aldhlal transcriptional activity as a homodimer and collagenase TRE as heterodimer with c-Fos. Transient transfection analysis with c-Jun dominant negative proteins (TAM67) further confirmed that activation of Aldh1a1luciferase reporter activity by acrolein is dependent on c-Jun (Figure 16).

Transient transfection experiments by co-transfection of Aldh1a1 5'-flanking luciferase constructs and c-Jun, JunD or JunB expression plasmids in HepG2 demonstrates that many members of the jun family proteins activate Aldh1a1-luciferase activity, with c-Jun serving as a more effective transcription factor (Figure 17). We also noticed that while Fra1 and Fra2 had no significant effect on c-Jun-mediated transactivation of Aldh1a1-luciferase activity, over-expression of c-Fos modestly inhibited activation of Aldhla1 reporter activity by c-Jun (Figure 19). Abrogation of cJun-dependent gene activation by c-Fos was previously reported by Marden et al, who demonstrated that whereas c-Jun homodimers strongly activated CYP $2 J 2$ expression, heterodimers formed between c-Fos and c-Jun were not active (Marden et al., 2003). Moreover, previous studies also demonstrated that the c-Jun-mediated activation of the human atrial natriuretic peptide (ANP) promoter was inhibited by overexpression of $c-$ Fos (Kovacic-Milivojevic and Gardner, 1992).

The effects of increasing concentrations of c-Fos on the activation of Aldhlal- or human collagenase TRE-luciferase activity by c-Jun was used to further assess the role of c-Fos on c-Jun-mediated transactivation of Aldhlal gene (Figure 19). A concentrationdependent activation and inhibition of collagenase TRE- and Aldh1a1-luciferase activity, respectively, by c-Fos was noted, indicating that the predominant $\mathrm{AP}-1$ complex that causes enhanced Aldh1a1 promoter luciferase activity is c-Jun homodimer and not c- 


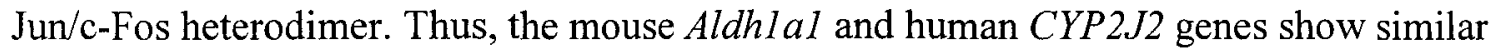
pattern of regulation by AP-1.

\section{The role of NF- $\mathrm{KB}$ signaling pathways in BHA or acrolein-mediated activation of} Aldh1a1 gene

Previous studies demonstrate that c-Jun can enhance transcription of target gene by functional interaction with other transcription factors, such as Nrf2 and NF- $\mathrm{kB}$. The up-regulation of human $C Y P 2 J 2$ gene by BHA was shown to be mediated by interaction of c-Jun with Nrf2 (Lee and Murray, 2010). Thus, Nrf2 apparently acts as a binding partner for c-Jun transcriptional activation of CYP2J2 gene. However, transient transfection experiment establishes that over-expression of $\mathrm{Nrf} 2$ had no effect on c-Junmediated activation of Aldh1a1-luciferase activity (Figure 21). Acrolein and other electrophiles have been reported to enhance the expression of cytoprotective genes by activation of nuclear factor $\mathrm{kB}(\mathrm{NF}-\mathrm{kB})$ transcription factor (Pinkus et al., 1996). Moreover, AP-1 interacts with NF- $\mathrm{kB}$ to activate gene transcription (Rahmani et al., 2001). TNF $\alpha$ is a pro-inflammatory cytokines activated by cytotoxic aldehydes and is a strong activator of the NF- $\mathrm{kB}$ signaling pathway. The concentration $(1 \mathrm{ng} / \mathrm{ul})$ of TNF $\alpha$ that normally stimulate the NF-kB responsive luciferase activity could not activate Aldh1a1-luciferase activity (Figure 22). However, TNF $\alpha$ inhibited c-Jun-mediated activation of Aldh1a1-luciferase activity, suggesting that NF- $\mathrm{kB}$ activation abrogates cJun induced activation Aldhlal activity. 


\section{Role of MAPKs in electrophile-mediated transcription of Aldh1a1}

Lipid aldehydes were previously shown to induce the expression of electrophile detoxifying genes by activation of MAPK such as JNK and ERK (Lamb et al., 2003;Wu et al., 2006). Hepa1c1c7 cells treated with acrolein $(20 \mu \mathrm{M})$ showed increase JNK phosphorylation $2 \mathrm{~h}$ after treatment compared to untreated control (Figure 10). Experiments using specific inhibitors of JNK, ERK or MEK1 revealed the functional importance of MAPK activation for Aldh1a1 gene expression. The treatment of HepG2 with JNK (SP600125) or ERK/MEK1 (PD98059) inhibitors blocks acrolein or c-Junmediated transactivation of Aldh1al promoter activity (Figure 22). However, the phosphatase 2A inhibitor (okadaic acid) had no significant effect on acrolein or c-Junmediated transactivation of Aldh1a1 luciferase activity. These results suggest that JNK plays a critical role in electrophile-induced transcription of Aldh 1 al and shows functional consequences of increased phosphorylation of c-Jun and JNK by acrolein.

In summary, we have provided evidence that Aldh1a1 gene expression can be induced by electrophiles such as acrolein and BHA and that increased expression of Aldh1al gene by electrophiles is dependent on c-Jun/AP-1 and not Nrf2 or NF-kB. Aldhlal gene expression was modulated by a c-Jun homodimer, but not by c-Jun/c-fos heterodimers. The MAPK especially JNK also plays a crucial role in electrophilemediated transactivation of Aldhlal gene. 


\section{CHAPTER IV}

\section{CHARACTERIZATION OF CIS-RESPONSIVE DNA ELEMENT INVOLVED IN REDOX-PROTECTIVE TRANSCRIPTION OF ALDH1A1 GENE}

\section{INTRODUCTION}

The comparison of the sequences of the proximal promoter region of mouse Aldh1a1 (m-AHD-2) revealed similarities with the rat RALDH1A1 and the human hALDH1A1 (Guimond et al., 2002;Hsu et al., 1999). Analysis of the 5'-flanking region of the mouse Aldh1a1 for putative regulatory elements revealed an Oct 1 binding site at $68 \mathrm{bp}$ and a CCAAT box at $-87 \mathrm{bp}$ relative to the transcription start site, which are well conserved in the rat RALDH1 and human ALDH1 genes. Transient transfection studies using rat RALDH1 promoter deletion luciferase reporter constructs demonstrated that CCAAT and Oct motifs were essential for basal promoter activity (Guimond et al., 2002). The conservation of these motifs in the mouse Aldh1al and human ALDH1A1 promoter suggests that these regulatory elements might also be critical for the basal promoter activity of mouse Aldh1al and human ALDH1A1. The analysis of nucleotide sequence of the mouse Aldhla1 promoter region, starting from -1963 to +25 relative to the transcription start site revealed a number of potential regulatory elements, such as GATA, E2F, AhR-Arnt, Stat5, Sox5, SP1 and NF- $\kappa B$ binding sites. Previous studies identified a putative retinoic acid response element (RARE) located at $-91 /-75$ bp adjacent to the CCAAT box of the hALDH1 gene (Elizondo et al., 2000;Elizondo et al., 2009). The 
RARE sequence was later identified at a similar position in mouse Aldh1 promoter and was reported to mediate retinoic acid (RA) down-regulation of hALDH1 and mouse Aldh1al gene expression through interaction of the RAR and C/EBP $\beta$ in human (HepG2) and mouse (Hepa1) hepatoma-derived cells (Elizondo et al., 2009), respectively. The down-regulation of ALDH1A1 by elevated hepatic RA is a feedback pathway to control RA biosynthesis since ALDH1A1 is a major enzyme involved in the biosynthesis of RA. Aldh1al is highly expressed in the liver and lens which are suggested to generate large amounts of ROS and cytotoxic lipid aldehydes, such as HNE and acrolein (Marchitti et al., 2008; Vasiliou et al., 2004). It is postulated that increased expression of Aldhlal in these organs is a mechanism to protect against aldehyde-induced oxidative damage and oxidative stress-induced pathologies. We also showed that Aldhlal efficiently metabolizes lipid-derived aldehydes and protects liver-derived cell lines from the toxic effect of lipid aldehydes. The induction of Aldhlal gene expression by high levels of acrolein might represent a mechanism to control the levels of acrolein in the body by enhanced metabolism and detoxification. Thus, an understanding of the factors controlling Aldh1al expression may assist efforts to control the progression of steatosis to steatohepatitis.

We demonstrated that electrophiles, such as BHA and acrolein induce the expression of Aldh1a1 gene by activation of c-jun/AP-1 proteins. However, it is still not clear how AP-1 modulate Aldhlal gene expression by electrophiles. The regulation of Aldhlal gene expression by AP-1 is similar to that of Cyp2J2 gene but different from the human collagenase gene. The AP-1 proteins are composed of either homodimers of jun family (c-Jun, JunD and JunB) or heterodimers with fos family (c-fos, Fos-B, Fral and 
Fra2). One of the well-characterized AP-1 target genes is the human collagenase gene, which contains a consensus TRE (TGAC/GTCA). Tumor-promoting phorbol $12-$ myristate 13-acetate (TPA) was shown to activate the transcription of human collagenase gene by promoting the association of c-Jun/c-Fos heterodimer to its cognate TRE. The AP-1 (c-jun/c-fos) transcription factors are also known to recognize the half site (underlined) of the ARE (A/GTGACNNNGC) and are activated during oxidative stress and by electrophiles. Hasselblatt et al demonstrate that c-Jun binds to the nos 2 promoter in vivo and is required for efficient expression of inducible nos gene during Con A- or LPS-mediated hepatitis (Hasselblatt et al., 2007). Moreover, a previous study in HepG2 cells demonstrates that the up-regulation of $C Y P 2 J 2$ gene transcription by BHA was dependent on the binding of c-Jun and Nrf2 to an atypical AP-1-like element in the proximal promoter of CYP2J2 (Lee and Murray, 2010). Ethanol was shown to induce the expression of the TFIIIB components, Brf1 and TATA-binding protein (TBP) by promoting a marked increase in the direct recruitment of c-Jun to TBP and Brf1 (Zhong et al., 2011). Therefore, we hypothesized that electrophiles induce the expression of Aldhlal gene by promoting the binding of c-Jun to its cognate cis-acting regulatory elements in the promoter of Aldhlal gene.

Analysis of the 5'-flanking region of the mouse Aldh1al gene using the Genomatrix MatInspector Professional consensus sequence identification program indicates the presence of four AP-1-like elements at positions -1516 (GCTGAATCA), 1069 (GCTCAGTCA), -758 (ATGATTCA) and -60 (ATGACCCT). Luciferase reporter constructs containing Aldh1a1 promoter region with these AP-1-like binding sites were shown to be responsive to c-Jun homodimers and not c-Jun/c-fos heterodimers. A 
number of AP-1-responsive genes contain atypical AP-1-like elements that accommodate alternate combination of bZIP factors and this can explain the difference in the modulation of Aldh1a1 and collagenase gene expression by AP-1 transcription factors.

In this chapter, we characterized the cis-regulatory DNA element involved in redox-protective transcription of Aldhlal gene. Furthermore, we assessed in vivo whether BHA exposure promotes the binding of c-Jun/AP-1 to the putative AP-1-like binding sites in the proximal promoter of mouse Aldhlal gene.

\section{EXPERIMENTAL DESIGN AND METHODS}

\section{Cloning of Aldh1a1 deletion plasmids.}

Mouse genomic DNA was purchased from Promega (Madison, WI) and used in PCR experiments to generate 2002 bp of mouse 5'-flanking sequence as described above in Chapter 3. This region contains $-1963 /+23$ bp relative to the transcription start site (TSS) of mouse Aldhlal gene. We generated three (3) deletion constructs; 1534 bp (1496/+27), 1043 bp (-1005/+27) and 518 bp $(-480 /+27)$ fragments of the mouse Aldh1a1 promoter by PCR using the $-1963 /+23$ bp as template. The downstream primer for synthesis of the three deletion constructs was similar to that of the $2002 \mathrm{bp}$ fragment (Chapter 3). The following upstream primers, which introduced a Nhe1 site, were used to generate the PCR products for the deletion constructs.

1534 bp: 5'-GCTAGCCATGGATCTGGCTGGATCTG-3.

1043 bp: 5'-GCTAGCGGAGGTGGCAATTTCACTAC-3'.

518 bp: 5'-GCTAGCGGTTTGCTGGTAGCCATGTT-3'. 
The PCR products were recovered into a pCR2.1 vector (Invitrogen, Carlsbad CA). The sequences of the PCR products were confirmed by sequence analysis in the University of Louisville, Center for Genetics and Molecular Medicine Nucleic Acid core facility. Nhe1 and HindIII were used to subclone the $1534 \mathrm{bp}, 1043 \mathrm{bp}$, and $518 \mathrm{bp}$ Aldhla1 promoter fragments into pGL3-Basic to generate -1496/+27Aldh1a1-Luc, -1005/+27Aldh1a1-Luc and $-480 /+27$ Aldh 1 al-Luc constructs, respectively. A far upstream region of the Aldh1a1 promoter (-4673/-3000 bp) that contains putative ARE/TRE site was also generated using the upstream primer; 5'-GGTACCACTCAAATGGCTGAGCCAATG3' and downstream primer, 5'-GCTAGCACTCTTCCCATGGCTGTCTTG-3'. This 1684 bp PCR fragment was cloned upstream of the -1496/+27Aldh1a1 or -518/+27Aldh1a1Luc to generate the heterologous mouse Aldhlal constructs pGL1.5-1684Aldh1al and pGL0.5-1684Aldh1a1, respectively.

HepG2 cells were transfected using $1 \mu \mathrm{g} / 2.5 \mu \mathrm{L}$ of Lipofectamine LTX and $1 \mu \mathrm{L}$ of Plus (Invitrogen, Carlsbad, CA) with $250 \mathrm{ng} /$ well of pGL0.5-1684Aldhla1, pGL1.51684Aldh1a1, -1963/+27Aldh1a1, -1496/+27Aldh1a1-Luc, -1005/+27Aldh1a1-Luc and 480/+27Aldh1al-Luc. The cells were co-transfected with or without pRSV-cjun (50 ng). After overnight incubation at $37^{\circ} \mathrm{C}$, the transfection media was replaced with $1 \mathrm{ml}$ media containing $10 \%$ FBS. The cells were harvested after overnight incubation $(\approx 17-24 \mathrm{~h})$ with $100 \mu \mathrm{L}$ of Luciferase cell lysis buffer (Promega, Madison, WI). The $\beta$-galactosidase and luciferase activities were determined as described by Falkner et al (1998). The data were expressed as luciferase activity relative to $\beta$-galactosidase activity to normalize for transfection efficiency. All transient transfection experiments were performed in triplicate and experiments were repeated at least twice for confirmation. Statistical comparisons 
among treatment groups were determined using a two-tailed test, with $\mathrm{p}<0.05$ as a criterion for significance.

\section{Site-Directed Mutagenesis}

Mutagenesis of the two putative AP-1 sites located at -1069 and -757 bp of Aldh1a1 was carried out using GeneTailor ${ }^{\mathrm{Tm}}$ Site-Directed Mutagenesis systems (Invitrogen, Carlsbad, CA) following standard protocol. Briefly, the $-1496 /+27$ Aldh1a1 construct was methylated using DNA methylase at $37^{\circ} \mathrm{C}$ for $1 \mathrm{~h}$. The template strand is methylated so that it is marked for degradation by the host $\mathrm{Mcr} B \mathrm{C}$ endonuclease. The methylated plasmid was then amplified by PCR with two overlapping primers, one of which contains the target mutation using Platinum Taq Polymerase High fidelity. The PCR conditions were as follows: $94^{\circ} \mathrm{C}$ for $2 \mathrm{~min} ; 20$ cycles of $94^{\circ} \mathrm{C}$ for $30 \mathrm{~s} ; 55^{\circ} \mathrm{C}$ for $30 \mathrm{~s} ; 68^{\circ} \mathrm{C}$ for $7 \mathrm{~min}(\approx 6.5 \mathrm{~kb}$ plasmid $)$; and $68^{\circ} \mathrm{C}$ for $10 \mathrm{~min}$. The primers for mutagenesis of AP-1 sites located at -1069 and -758 bp are shown below.

-758 bp: Sense, 5'-TCGACACTGCTTAGAGTAATaATaaACAAGTGCACGC-3' Antisense, 5' ATTACTCTAAGCAGTGTCGAAGGAAAGAAT-3'

-1069 bp: Sense, 5'-TATTTACAAATTGAGAAGCTaAaTaAAGGCAAAAAGA-3' Antisense, 5'-AGCTTCTCAATTTGTAAATACAGAGAGGAA-3'

The PCR product, linear and double-stranded was analyzed by a $1 \%$ agarose gel stained with ethidium bromide. The mutagenesis mixture was then transformed into One-Shot ${ }^{\mathrm{R}}$ MAX Efficiency DH5 $\alpha^{\mathrm{TM}}-\mathrm{T} 1^{\mathrm{R}} E$. coli. The host $E$. coli circularized the linear mutated DNA and McrBC endonuclease in the host cell then digested the methylated template DNA, leaving only the unmethylated and mutated product. The sequences of the recovered PCR products were analyzed for mutagenesis by sequence analysis in the 
University of Louisville, Center for Genetics and Molecular Medicine Nucleic Acid core facility.

\section{Preparation of Nuclear Extracts}

Nuclear extracts were prepared from mouse liver and HepG2 cells transfected with either pcDNA3.1 (vector control; $2 \mu \mathrm{g} /$ well) or pcDNA3.1-c-Jun (c-Jun expression plasmid; $2 \mu \mathrm{g} /$ well) for $48 \mathrm{~h}$ using NE-PER nuclear and cytoplasmic extraction reagents (Pierce, Rockford, IL) according to standard protocol. Briefly, HepG2 cells $\left(1 \times 10^{6}\right)$ in $35 \mathrm{~mm}$ dishes were washed twice with ice-cold PBS and then scraped from the dishes with $1 \mathrm{ml}$ of PBS and transferred to microcentrifuge tubes. Cells were then centrifuged at $1500 \mathrm{~g}$ for $5 \mathrm{~min}$. The supernatant was discarded and the cell pellet was allowed to swell after addition of ice-cold CERI with vigorous vortexing of the tube to suspend the cell pellet. After 10 min of incubation at $4^{\circ} \mathrm{C}$, ice-cold CERII was added and the tube was again vigorously vortexed and incubated for $1 \mathrm{~min}$. The tube was then centrifuged at $16000 \mathrm{xg}$ for $5 \mathrm{~min}$ and the cytoplasmic fraction was transferred to a clean tube. The insoluble nuclear pellet was suspended in ice-cold NER. The tube was then incubated in

ice for $40 \mathrm{~min}$ with $15 \mathrm{~s}$ vortexing every $10 \mathrm{~min}$. The tube was then centrifuged at $16000 \mathrm{~g}$ for $10 \mathrm{~min}$ and the nuclear extract was aliquoted and stored at $-80^{\circ} \mathrm{C}$ until used for EMSA. Protein concentration was determined by Pierce ${ }^{\circ}$ BCA Protein Assay (Pierce, Rockford, IL) using bovine serum albumin as standards.

\section{Electrophoretic Mobility Shift Assay (EMSA)}

A non-radioactive LightShift Chemiluminescent EMSA kit (Pierce, Rockford, IL) was used to examine whether c-Jun binds to the putative AP-1 binding sites on Aldh1a1 
proximal promoter. The oligonucleotides used as probes or competitors in gel shift assays were end-labeled at their $5^{\prime}$ with biotin and the sequences are shown below:

AP-1 Consensus: Sense, 5'-CTAGTGATGAGTCAGCCGGATC-3'

Antisense, 5'-GATCGATCCGGCTGACTCATCA-3'

AP-1 Site B: Sense, 5'- TGCTTAGAGTAATGATTCACAAGTGCACG-3'

Antisense, 5'-CGTGCACTTGTGAATCATTACTCTAAGCA-3'

AP-1 Site B Mut: Sense, 5'-TGCTTAGAGTAATaATaaACAAGTGCACG-3'

Antisense, 5'-CGTGCACTTGTttATtATTACTCTAAGCA-3'

AP-1 Site C: Sense, 5'-AATTGAGAAGCTCAGTCAAGGCAAAAAGA-3'

Antisense, 5'-TCTTTTTGCCTTGACTGAGCTTCTCAATT-3'

AP-1 Site C Mut: Sense, 5'-AATTGAGAAGCTaAaTaAAGGCAAAAAGA-3'

Antisense, 5'-TCTTTTTGCCTTtAtTtAGCTTCTCAATT-3'

The complementary oligonucleotides were annealed using a thermocycler (Applied Biosystems, GeneAmp PCR system 2400) program for annealing complementary oligonucleotides. EMSA reactions containing 20 fmoles of biotin-end labeled doublestranded probes and $5 \mu \mathrm{g}$ nuclear proteins (NP) were incubated for $20 \mathrm{~min}$ at room temperature in a $10 \mathrm{X}$ binding buffer with $5 \mathrm{mM} \mathrm{MgCl}_{2}, 2.5 \%$ glycerol, $1 \% \mathrm{NP}-40$ and $50 \mathrm{ng} / \mu \mathrm{l}$ poly $(\mathrm{dI}-\mathrm{dC})$. The specificity of binding was analyzed by excess of unlabeled double stranded probes included in the binding reactions. In the competition experiments, 25- to 100-fold excess of unlabeled double stranded EMSA probes was included in the binding reactions. To further confirm specificity of binding, a 100-fold excess of unlabeled double stranded probe with mutations at the AP-1 binding site was included in the binding reactions. For super shift analysis, nuclear extracts were incubated with 
rabbit polyclonal c-Jun (sc-44 X, Santa Cruz Biotechnology, CA), c-fos (sc-52 X) or Nrf2 (sc-722) antibodies for $30 \mathrm{~min}$ at $4^{\circ} \mathrm{C}$ before addition of the labeled probe. Loading buffer was added to the reactions and the protein-DNA complexes were resolved by electrophoresis on $6 \%$ precast DNA retardation gel (Invitrogen) in $0.5 \%$ TBE buffer $(90$ $\mathrm{mM}$ Tris- $\mathrm{HCl}, 90 \mathrm{mM}$ boric acid, $2.5 \mathrm{mM}$ EDTA) at $100 \mathrm{~V}$ for $1 \mathrm{~h}$ at $4^{\circ} \mathrm{C}$. Following electrophoresis, the binding reactions was then electrophoretically transferred onto a nylon membrane (Amersham Hybond- ${ }^{+}$, GE Healthcare) with $0.5 \%$ TBE for $1 \mathrm{~h}$ at $100 \mathrm{~V}$. At the end of transfer, the transferred DNA-protein complexes were then crosslinked onto membrane using a UV cross-linker that was set at $120 \mathrm{~mJ} / \mathrm{cm}^{2}$ for an exposure time of $2 \mathrm{~min}$. The biotin-labeled DNA was then detected using the Chemiluminescent Nucleic acid detection module (Pierce) following standard procedure for detection of immobilized nucleic acids.

\section{Chromatin Immunoprecipitation Assay (ChIP).}

C57BL/6 mice were placed on AIN76 diet (control) or diet containing $0.45 \%$ BHA for 7 days. Animals were sacrificed and intact nuclei from the livers of control or BHA-treated mice were purified by sucrose density gradient without disrupting the internal macromolecular interactions. The ChIP assay was performed using the MAGnify chromatin-immunoprecipitation system (Invitrogen, CA) according to manufacturer's protocol with minor modifications. The pure nuclei were suspended in PBS and fixed in $1 \%$ formaldehyde in PBS at room temperature to cross-link the DNA binding proteins to cognate $c i s$-acting elements. The nuclei were harvested after $30 \mathrm{~min}$, washed with PBS and solubilized in Buffer A containing $50 \mathrm{mM}$ Tris-Cl, pH 8.0, 1\% SDS, 5 mM EDTA, 5 mM EGTA, 0.5 mM PMSF and complete protease inhibitor mix (Roche Molecular 
Biochemicals). The homogenate was sonicated 6-7 times on ice at $40 \%$ setting (Branson sonicator) to shear the chromosomal DNA into fragments of $\approx 200$ to $500 \mathrm{bp}$ in size. The insoluble material was removed by centrifugation at $20,000 \mathrm{xg}$ at $4^{\circ} \mathrm{C}$ for $5 \mathrm{~min}$ and the soluble chromatin supernatant (chromatin extract) was stored at $-80^{\circ} \mathrm{C}$ until use. Before use, the chromatin extract was diluted 5-fold with dilution buffer containing $20 \mathrm{mM}$ Tris$\mathrm{Cl}, \mathrm{pH} 8.0,1 \%$ Triton X-100, $1.2 \mathrm{mM}$ EDTA and $150 \mathrm{mM} \mathrm{NaCl}$. The cross-linked protein-DNA fragments were immunoprecipitated by overnight incubation of diluted chromatin with antibodies against IgG (control), c-Jun (sc-44 X; $1 \mu \mathrm{g} / \mu \mathrm{l}$ ) or c-fos (sc-447 $\mathrm{X} ; 1 \mu \mathrm{g} / \mu \mathrm{l})$ conjugated to Dynabeads protein $\mathrm{A} / \mathrm{G}$. The immune complexes were washed sequentially with IP buffer 1 and 2, and the protein-DNA cross-links were reversed by heat treatment in reverse cross-linking buffer containing proteinase $\mathrm{K}$. The uncrosslinked DNA pulled down by the different antibodies was purified using the DNA purification magnetic beads. The purified DNA was analyzed by real-time PCR (ABI 7900HT Sequence Detector System, Applied Biosystems, Foster City, CA) with primers spanning the putative AP-1 sites B and C in the proximal promoter of Aldh1a1 gene. The sequences of the ChIP primers are shown below:

AP-1 Site B: Forward, $\quad$ 5'-GTT CCT TCC ATA TCT TGT GCT GGG-3'

Reverse, 5'-GAG GTG CGT GCA CTT GTG AAT CAT-3'

AP-1 Site C: Forward 5'-TCC TTC AAG GTC TGT GAC CAA AGC-3'

Reverse 5'-AAC AGG GAC CTG AGG AGT GTG TTT-3' 


\section{RESULTS}

\section{Identification of AP-1-like elements that mediate electrophile response in the}

proximal promoter of Aldhlal gene by deletion analysis

The analysis of the proximal promoter of Aldhlal by the Genomatrix MatInspector Professional software revealed four putative AP-1-like elements at positions $-1516,-1069,-758$ and -60 (Figure 24). The p2.0Aldh1a1 construct containing 1963 to +27 of Aldh1a1 proximal promoter was used as template for the generation of three deletion constructs: p1.5Aldh1a1 (-1496 to +27$)$, p1.0Aldh1a1 $(-1005$ to +27$)$ and p0.5Aldhla1 $(-480$ to +27$)$ with progressive loss of the AP-1-like elements. To identify the critical AP-1-responsive element required for transactivation of Aldh1a1 gene by cJun, HepG2 cells were co-transfected with the Aldh1a1 5'-flank luciferase reporter constructs containing progressive deletions of the AP-1-like elements and a plasmid encoding c-Jun protein. The deletion of the AP-1-like element at positions -1516 had no significant effect on c-Jun-mediated transactivation of Aldh1a1-luciferase activity (Figure 25). However, the deletion of the putative AP-1 sites at positions -1069 and -758 significantly decreased activation of Aldh1a1-luciferase activity by c-Jun. Thus, deletion analysis indicates that the region containing these two AP-1-like elements at positions $758(\mathrm{AP}-1$ site $\mathrm{B})$ and -1069 (AP-1 site $\mathrm{C})$ relative to Aldhla1 transcription start site is critically important for c-Jun-dependent transactivation of Aldh1a1 transcriptional activity. 


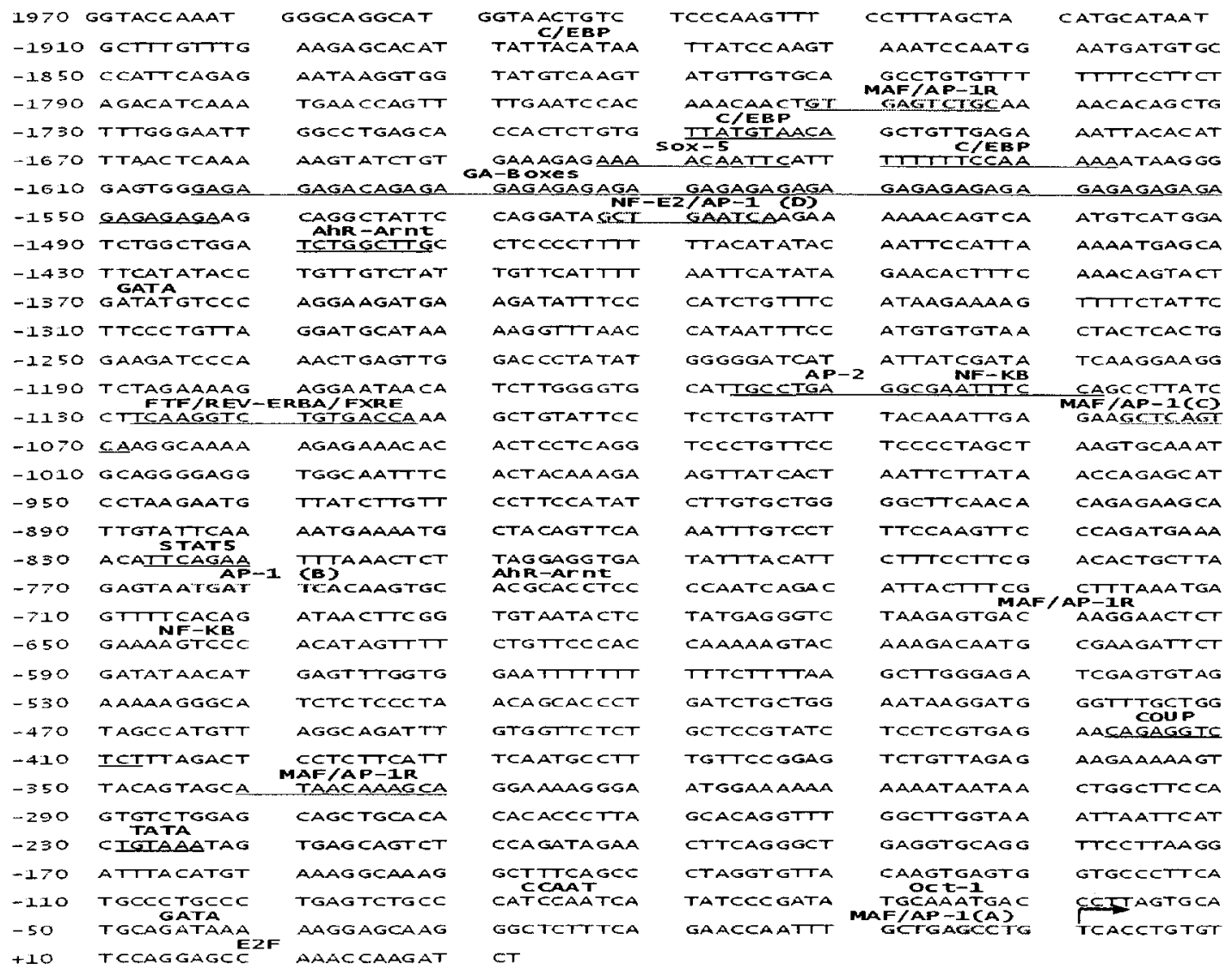

Figure 24. The nucleotide sequence of mouse Aldh1a1 promoter depicting potential AP-

1, Nrf2 (ARE) and NF- $\mathrm{kB}$ binding sites as well as other putative transcription factor (TF)

binding sites. The sequence shown is -1963 to +27 bp of mouse Aldhlal promoter. The underlined nucleotides represent putative TF binding sites identified by Genomatrix

MatInspector Professional consensus sequence identification software. Arrow indicates

the transcription start site $(+1)$. 

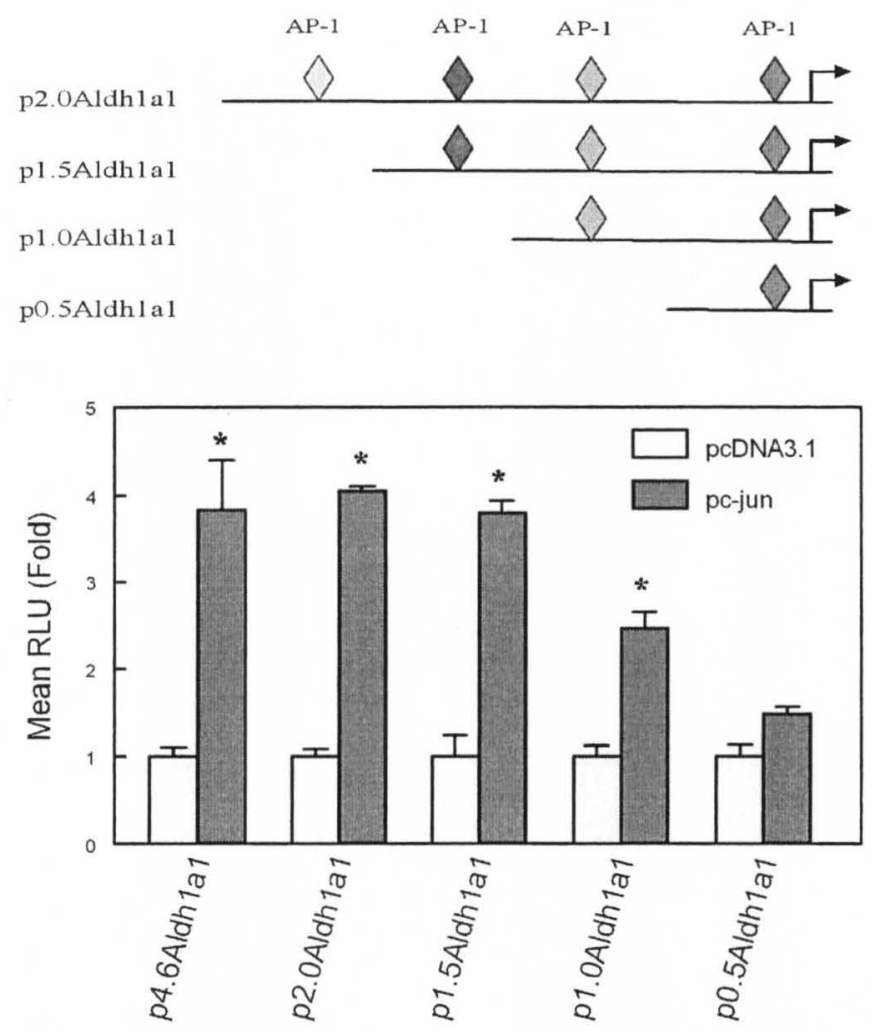

Figure 25. Deletion analysis to identify the AP-1-like responsive element in the proximal promoter Aldh1a1 gene. HepG2 cells were transiently co-transfected with 250 ng of 4673/+27Aldh1a1, -1963/+27Aldh1a1, -1496/+27Aldh1a1, -1005/+27Aldh1a1 or 480/+27Aldh1a1 luciferase construct and either pcDNA3.1 vector control or c-Jun (40 ng) expression plasmid. Luciferase activity was normalized to $\beta$-galactosidase activity and corrected for activities in cells co-transfected with empty plasmids. Data are means \pm SD from at least three independent experiments. * Significant induction compared to vector transfected cells $(\mathrm{p}<0.05 ; 2$ tailed $\mathrm{t}$ test). 


\section{Characterization of AP-1-like elements responsible for c-Jun-mediated transactivation of Aldh1a1 promoter activity by mutagenesis analysis}

To investigate the importance of the AP-1-like elements at -1069 and -758 in the activation of Aldh1a1-luciferase by c-Jun, these putative AP-1 elements were mutated by site directed mutagenesis. The functional consequences of the mutations were examined by transient transfection of the WT and mutant Aldh1a1-luciferase reporter constructs into HepG2 cells. The mutation of the proximal putative AP-1 element at position -758 (p1.5Aldh1a1MutB) resulted in $\approx 50 \%$ reduction in c-Jun-mediated activation of Aldh1a1 promoter activity compared to the p1.5Aldh1alWT (WT) constructs (Figure 26). However, mutation of the distal AP-1 sequence at position -1069 (p1.5Aldh1a1MutC) modestly decreased Aldhla1-luciferase activity compared to the WT plasmid. Double mutation of the putative AP-1 elements at sites B and C in the promoter of Aldh1al gene attenuated c-Jun responsiveness. These results indicate that both AP-1-like elements at sites B and C may be essential for c-Jun dependent transactivation of Aldh1a1 gene with the $\mathrm{AP}-1$ at site $\mathrm{B}$ playing a pivotal role. 


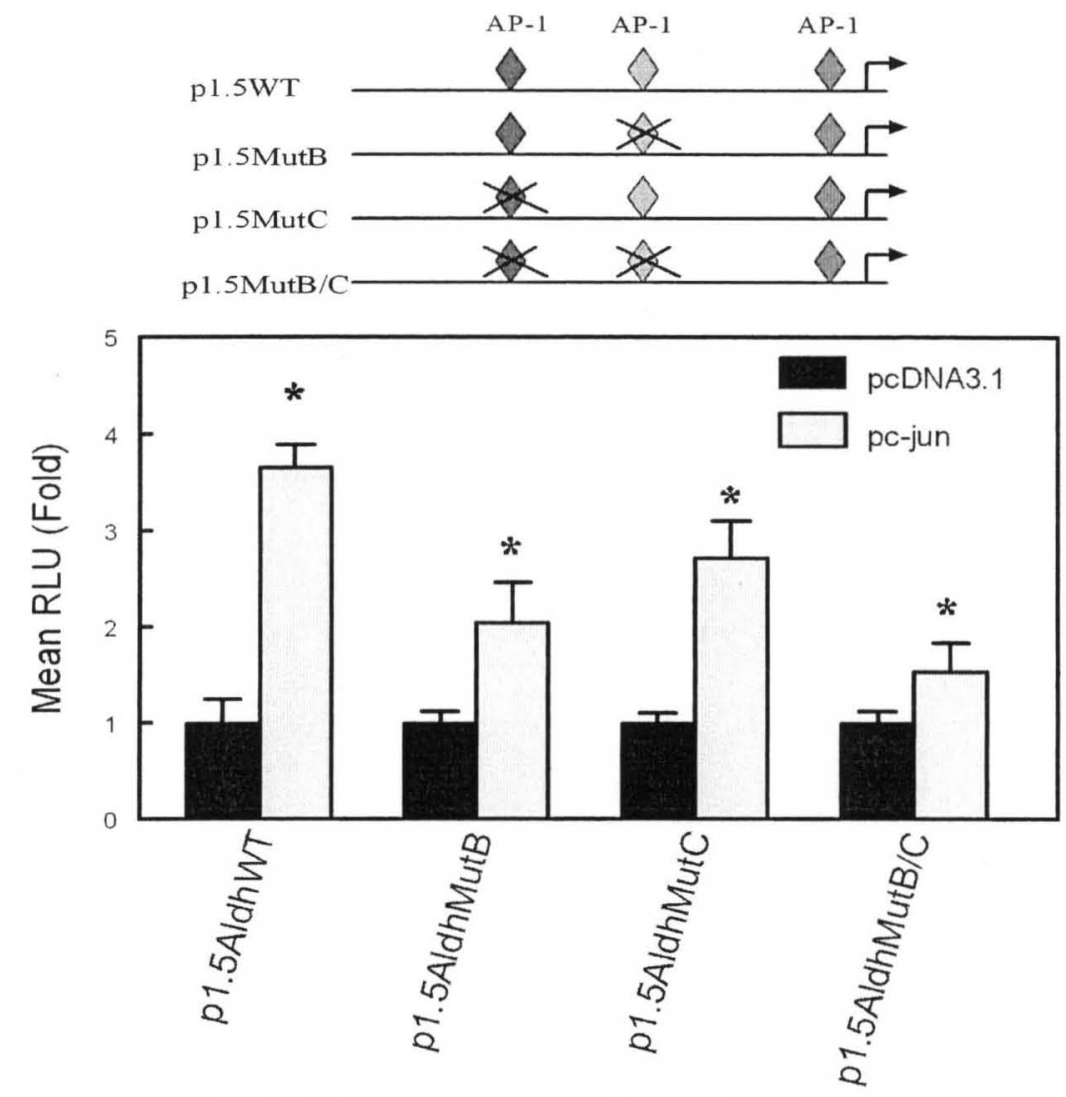

Figure 26. Mutagenesis of the putative AP-1-like elements located at -1069 bp (C site) and -758 bp (B site) on Aldh1a1 promoter activity. HepG2 cells were transiently cotransfected with $250 \mathrm{ng}$ of p1.5Aldh1a1WT (-1496/+27Aldh1a1), p1.5Aldh1a1MutB, p1.5Aldh1a1MutC or p1.5Aldh1a1MutB/C luciferase constructs and either pcDNA3.1 or c-Jun (40 ng) expression plasmids. Luciferase activity was normalized to $\beta$-galactosidase activity and corrected for activities in cells co-transfected with empty plasmids. Data are means \pm SD from at least three independent experiments. * Significant difference compared to c-Jun and p1.5AldhWT co-transfected cells ( $\mathrm{p}<0.05 ; 2$ tailed t test). 


\section{EMSA and super shift analysis to assess binding of AP-1 nuclear proteins to AP-1- like elements in the proximal promoter of Aldh1a1 gene}

We next wanted to establish whether AP-1 proteins bind to these putative AP-1like elements at sites B and C by EMSA. The nuclear extract isolated from HepG2 cells transfected with plasmid encoding c-Jun (Figure 27) was incubated with biotin-labeled double-stranded probes containing AP-1-like elements B and C. We observed formation of a DNA-protein complex when labeled probe spanning AP-1-like element B was incubated with nuclear extract but not with site C (Figures 28, 29 and 30). The specificity of nuclear proteins binding to the AP-1-like element B was examined by competition with cold unlabeled WT probe and unlabeled mutant probe containing mutations at the AP-1-like element at site B. The nuclear proteins present in HepG2 cells overexpressing c-Jun were allowed to form complexes with unlabeled WT (25- to 100-fold) or mutant probe (100-fold) prior to the incubation with biotin-labeled probes spanning AP-1-like element at site $\mathrm{B}$. The results demonstrate that 100 -fold excess concentration of unlabeled WT probe completely competed with the biotin-labeled probe containing site B for AP-1 binding proteins (Figure 28). Thus, excess unlabeled specific WT probe completely inhibited the formation of complexes with the biotin-labeled probe containing AP-1 element at site B. However, pre-incubation with unlabeled mutant probe only slightly competed with the biotin-labeled AP-1B site for protein binding (Figure 29). We then examined whether an increase in the concentration of nuclear extracts might result in protein binding to biotin-labeled probe generated from AP-1-like element at site C. 


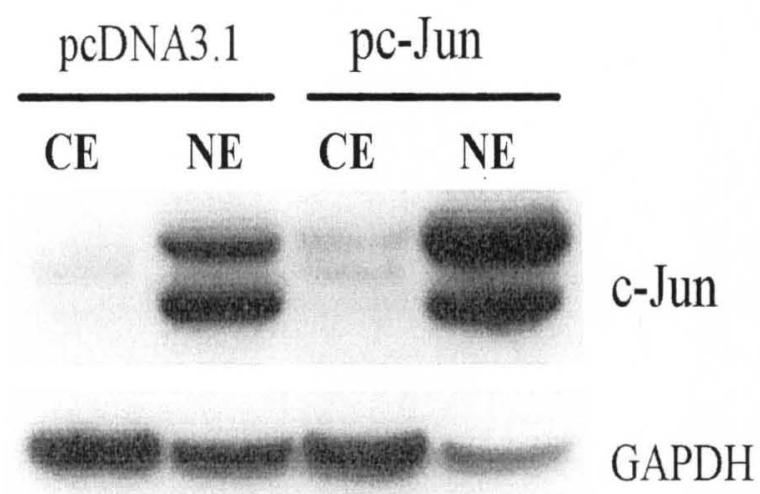

Figure 27. Western blots indicating over-expression of c-Jun in the nuclear extracts of HepG2 cells. Nuclear extracts were prepared from HepG2 cells transfected with either pcDNA3.1 (vector control; $2 \mu \mathrm{g} /$ well) or pcDNA3.1-c-Jun (c-Jun expression plasmid; 2 $\mu \mathrm{g} /$ well) for $48 \mathrm{~h}$ using NE-PER nuclear and cytoplasmic extraction reagents (Pierce, Rockford, IL) according to standard protocol. $48 \mathrm{~h}$ after transfection cell extracts were separated on SDS-PAGE gel and transferred onto nitrocellulose membranes. The membranes were probed with antibodies against c-Jun (1:1000; sc-45) and GAPDH (1:10000). Western blot experiment was performed in triplicate and the figure is a representative blot. 


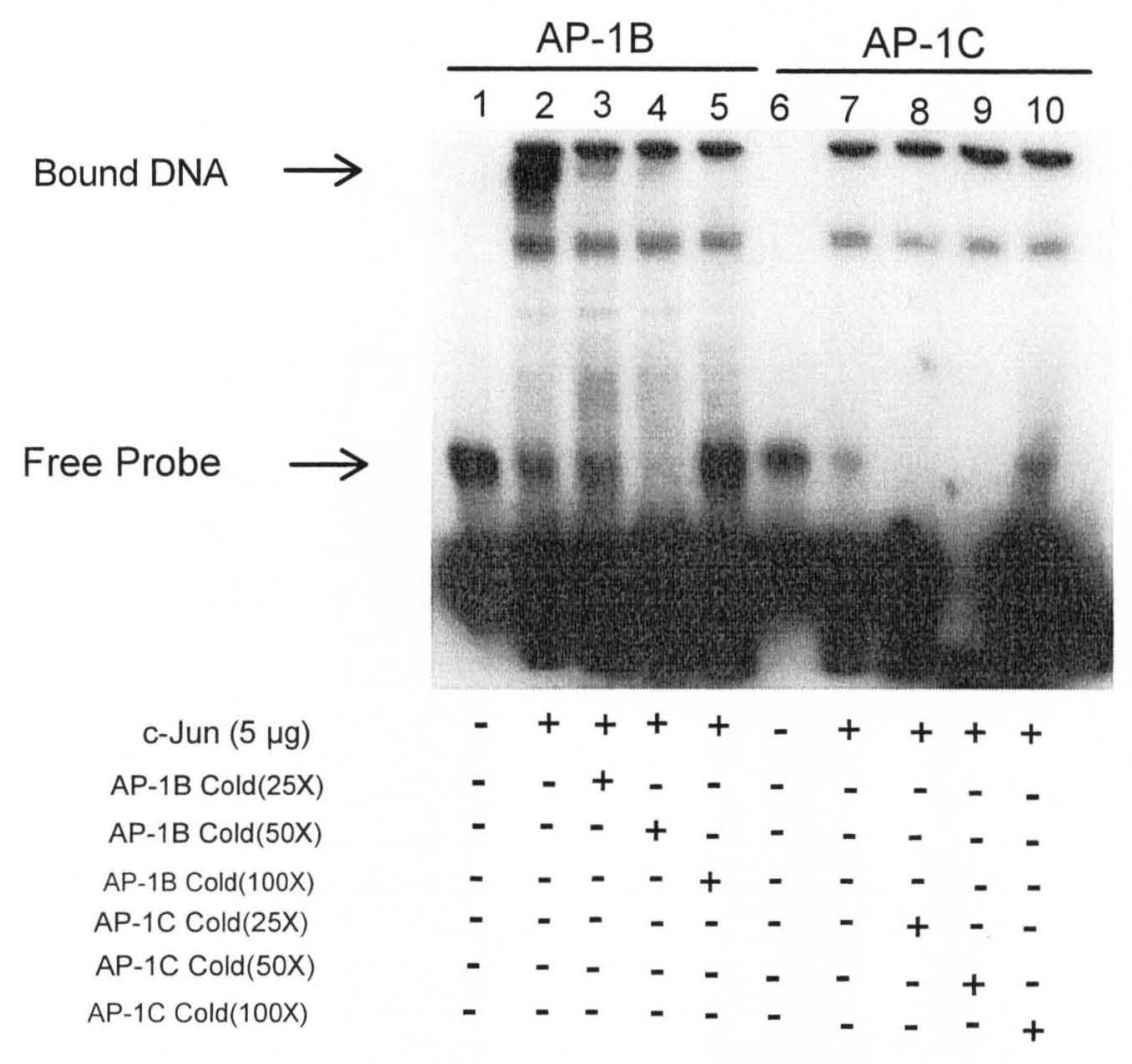

Figure 28. EMSA analysis indicates the formation of nuclear protein complex with the AP-1-like element at site B (-758) but not with the AP-1-like element at site C (-1069). Biotin-labeled double stranded probes were incubated with $5 \mu \mathrm{g}$ of nuclear extracts from HepG2 transfected with c-Jun expression plasmids for $20 \mathrm{~min}$. In the competition experiments, 25-100-fold excess of unlabeled double stranded probes was included in the binding reactions. The protein-DNA complexes were resolved by electrophoresis on $6 \%$ precast DNA retardation gel and the binding reactions were then electrophoretically transferred onto a nylon membrane. The immobilized biotin-labeled DNA was then detected using the Chemiluminescent Nucleic acid detection kit. The result shown in this figure was identical to that of three other independent experiments. 


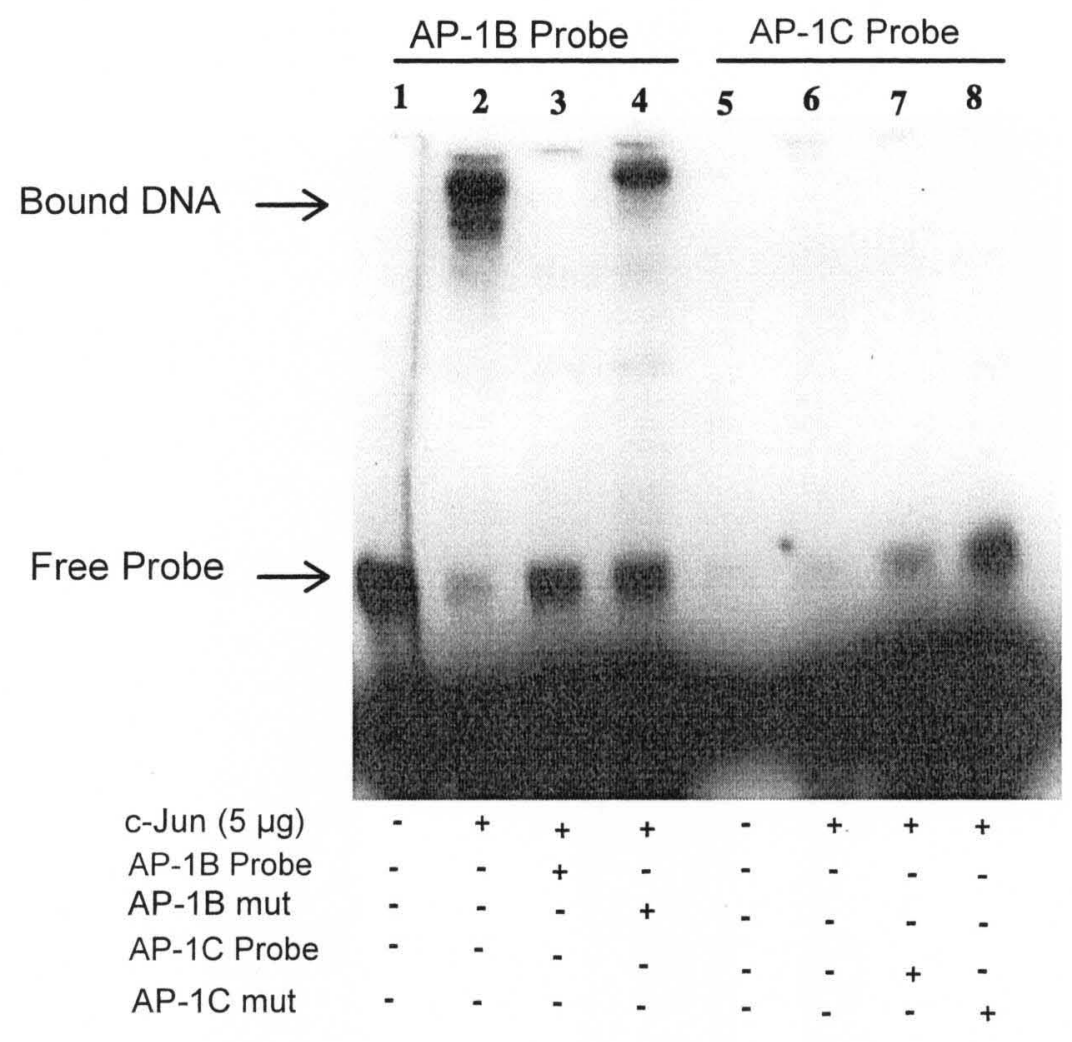

Figure 29. The nuclear protein complex with the AP-1-like site at B (-758) was competed by excess unlabeled wild type probe but not probe containing mutation in the AP-1-like element. The specificity of binding to the AP-1 site at B was further confirmed with 100fold excess of unlabeled double-stranded probe with mutations at the AP-1-like site at B. EMSA was performed with nuclear extracts from HepG2 cells over-expressing c-Jun as in Figure 28. The result shown in this figure was essentially identical to that of three other independent experiments. 
However, EMSA result shown in Figure 30 indicate the absence of nuclear protein complex at the AP-1-like element at site $\mathrm{C}$ even when incubated with high concentration of nuclear extracts $(10 \mu \mathrm{g})$ from c-Jun overexpressing HepG2 cells. These results indicate that the nuclear proteins do not form complexes with the AP-1-like element at site $\mathrm{C}$.

To identify the composition of the nuclear proteins that form complexes with the $\mathrm{AP}$-1-like element at site $\mathrm{B}$, super shift assays were performed by incubation of proteinDNA complex with antibodies against c-Jun, c-fos or $\mathrm{Nrf} 2$ at $4^{\circ} \mathrm{C}$ for 30 min (Figure 31). Incubation of nuclear protein-DNA complexes with antibodies against c-Jun but not c-fos or Nrf2 blocked the formation of the nuclear protein-DNA complex. The absence of nuclear protein complex with AP-1 site B instead of a super shift might be as a result of the $6 \%$ polyacrylamide gel used in our experiment. The antibody-nuclear protein-DNA complex may be too large to get into the gel. Future experiments will examine whether this problem can be resolved by using lower \% gels, such as $4-5 \%$ gel with a longer run time. Moreover, the absence of nucleoprotein complex at site B might also be due to cJun antibody binding to the DNA-binding domain of the c-Jun, thereby blocking the interaction between c-Jun and DNA. 


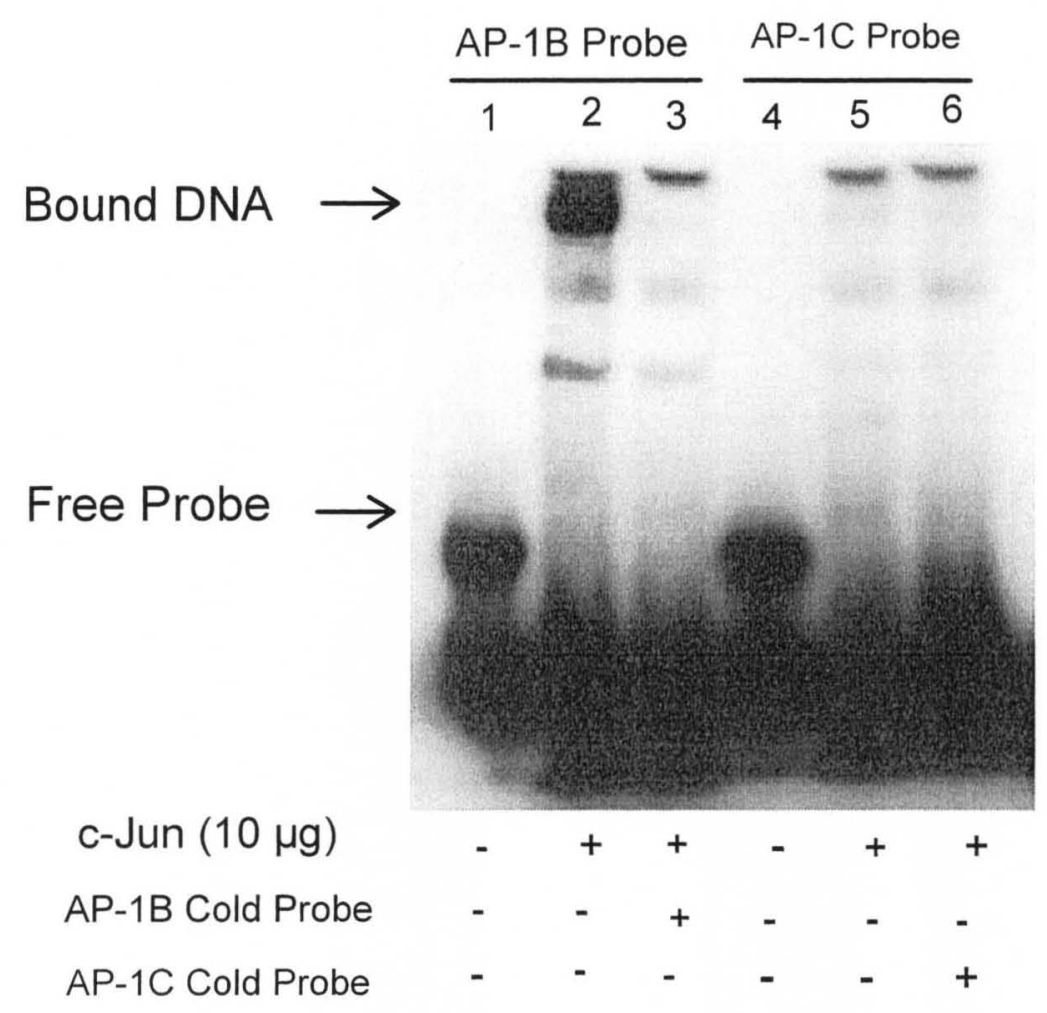

Figure 30. EMSA analysis indicates no protein binding to the AP-1 site C (-1069) at higher concentrations of nuclear extracts. The experiment was performed essentially as in Figure 28. However, biotin-labeled double stranded probes were incubated with $10 \mu \mathrm{g}$ of nuclear extracts for $30 \mathrm{~min}$. The figure is a representative of three other independent experiments. 


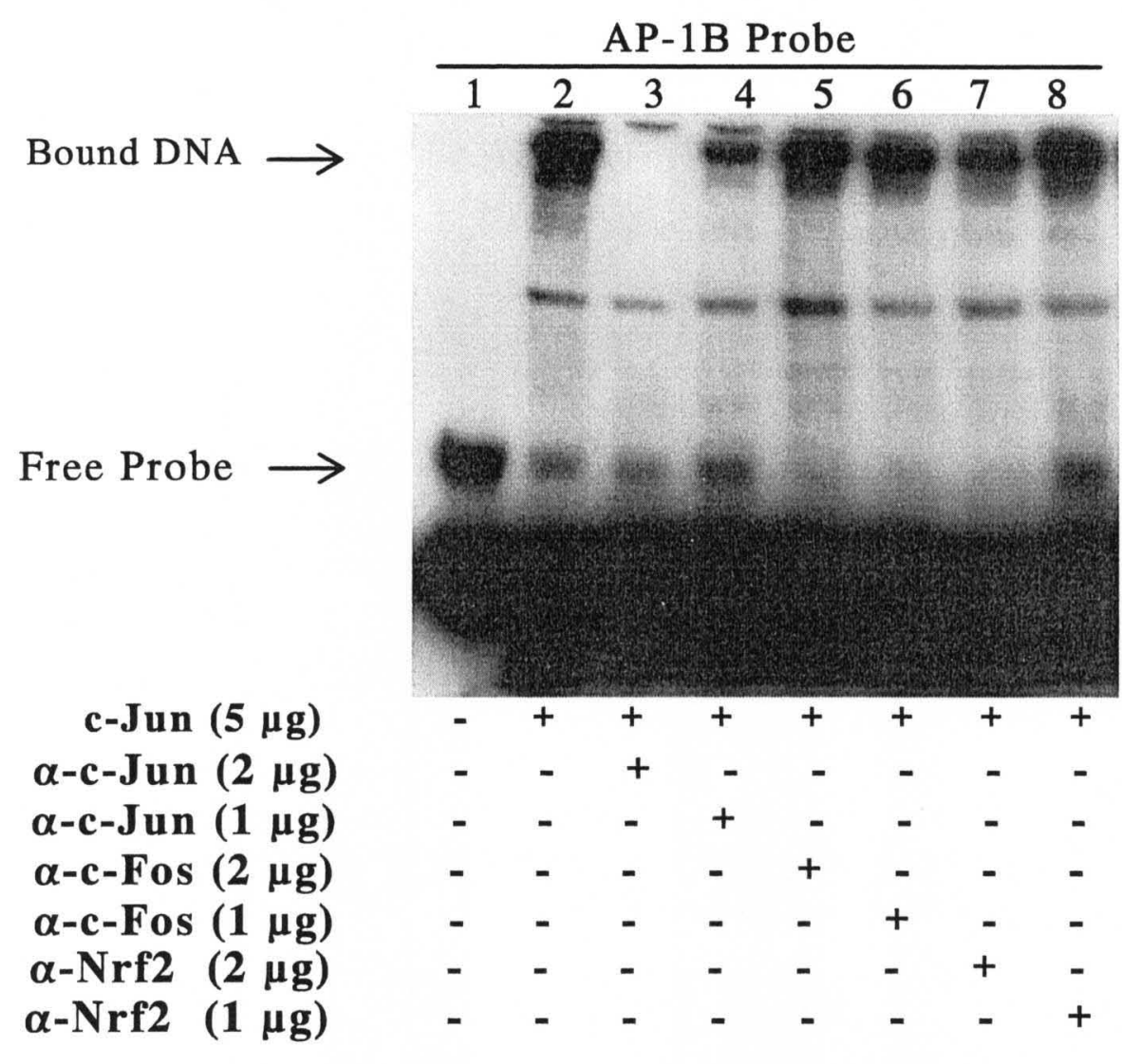

Figure 31. Super shift analysis confirms that a protein in the nuclear extract that binds to the AP-1 site B is c-Jun and not c-fos or Nrf2. EMSAs were performed by incubating nuclear extracts from HepG2 cells transfected with c-Jun encoding plasmids with biotin labeled probe containing AP-1B site of the mouse Aldh1a1 promoter. In super shift experiments, the nuclear extracts were incubated with rabbit polyclonal c-Jun, c-fos and Nrf2 antibodies for $30 \mathrm{~min}$ at $4^{\circ} \mathrm{C}$ prior to incubation with the labeled probe. The figure is a representative of three other independent experiments. 


\section{ChIP experiment to assess in vivo binding of nuclear proteins to putative AP-1 sites}

\section{in the proximal promoter of Aldh1a1 gene}

To investigate the functional importance of the AP-1-like sequences at sites $\mathrm{B}$ and $\mathrm{C}$ in the proximal promoter of Aldhlal gene, ChIP experiment was used to assess whether BHA promotes recruitment of c-Jun to these putative AP-1 binding sites at B and C. Using c-Jun or c-fos antibodies, the complexes containing cross-linked AP-1 proteins to the cognate AP-1 elements were pulled down from chromatin extracts prepared from the liver of control or BHA-treated mice. The DNA fragments were then analyzed by qRT-PCR with primers spanning the AP-1-like elements at B or $\mathrm{C}$ in the proximal promoter of Aldhlal gene. As shown in Figure 32, liver extracts from BHA-treated mice showed $\approx 10$-fold increase recruitment of c-Jun to AP-1-like element at site B compared to $\mathrm{IgG}$ control. The recruitment of $\mathrm{c}-\mathrm{fos}$ to the AP-1 element at site B in BHA-treated mice though higher than in control mice was comparable to that of $\operatorname{IgG}$ control. These results confirm that BHA promotes enhanced binding of c-Jun to the AP-1 B site in the proximal promoter of Aldhlal, which is associated with increased transcription. The recruitment of c-Jun to AP-1-like element at site $\mathrm{C}$ was similar to that of site $\mathrm{B}$. However, this result differs from our EMSA experiment, demonstrating the absence of nuclear protein complexes with the AP-1-like sequence at site $\mathrm{C}$. This disparity might be due to the close proximity of the AP-1-like elements at B and C, which are only $300 \mathrm{bp}$. The sonication method employed for DNA shearing in the ChIP experiment generated DNA fragments of $\approx 500 \mathrm{bp}$. Thus, inefficient shearing of the DNA could produce a single DNA fragment containing both AP-1-like elements. 
mALDH Site B

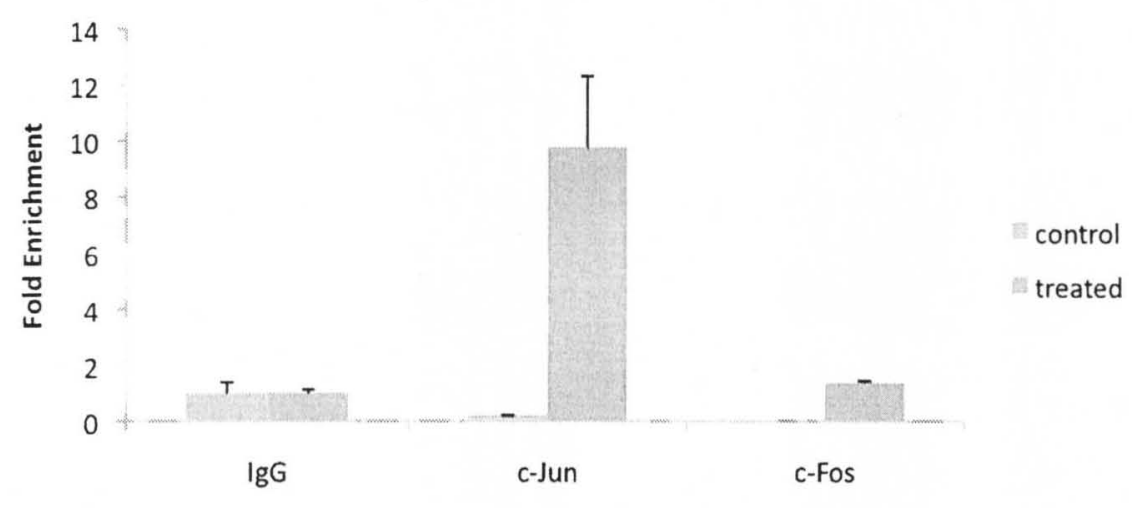

\section{mALDH - Site C}

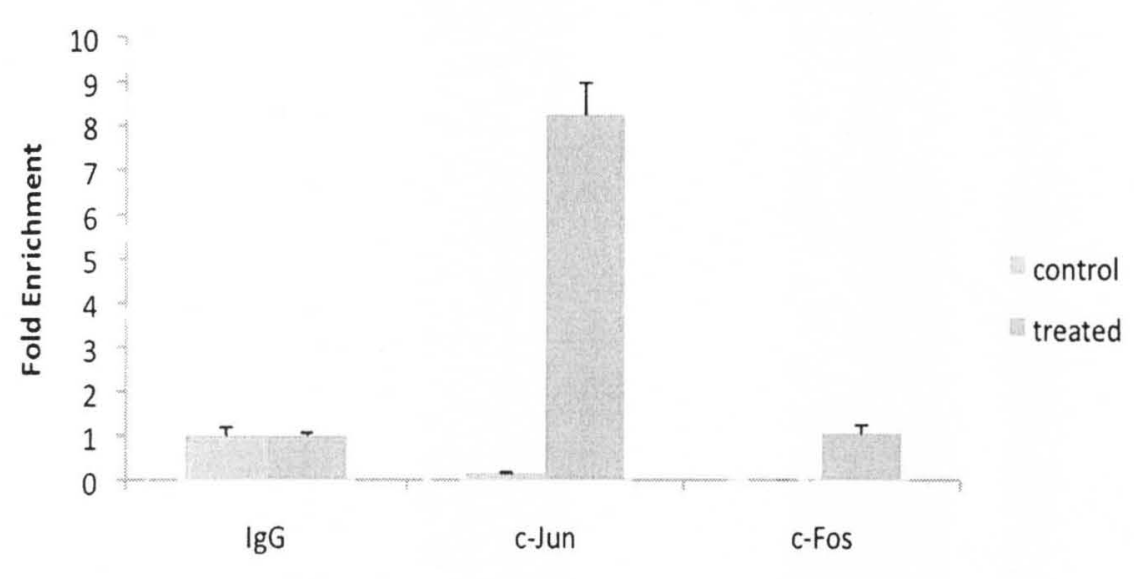

Figure 32. ChIP analysis to examine in vivo binding of c-Jun and c-fos to the AP-1 at sites B and C. Chromatin were prepared from the liver of control or BHA-treated mice. The complexes containing cross-linked DNA binding proteins to their cognate cis-element were pulled down with antibodies against IgG, c-Jun or c-fos conjugated to Dynabeads protein A/G. The purified DNA was analyzed by real-time PCR with primers spanning AP-1 sites B in the proximal promoter of Aldh1a1 gene. 


\section{DISCUSSION}

Due to the potential metabolism, electrophile detoxification and anti-apoptotic role of Aldh1a1 in mouse liver, an understanding of the molecular mechanism underlying Aldh1a1 regulation is of utmost importance, and could be of therapeutic importance in prevention of oxidative stress-induced pathologies. Aldh1a1 gene expression is modulated by electrophiles, such as acrolein and BHA, which are associated with enhanced expression of Aldhlal gene and transcriptional activation of c-Jun/AP-1. Transient transfection studies in HepG2 demonstrate that Aldh1a1 gene is activated by cJun homodimers, but not by c-Jun/c-fos heterodimers. The transcription factors, Nrf2 and $\mathrm{NF}-\mathrm{\kappa B}$ were not involved in electrophile-induced transcription of Aldhlal gene. Previous studies indicate that the up-regulation of cytoprotective genes by electrophiles are mediated by enhanced recruitment of redox-sensitive transcription factors to the cisresponsive element in the promoter of these genes. The up-regulation of $C Y P 2 J 2$ gene transcription by BHA is dependent on the binding of c-Jun and Nrf2 to an atypical AP-1like element in the proximal promoter of CYP2J2 (Lee and Murray, 2010). The induction of rat glutathione synthetase gene expression by tBHQ is mediated by a regulatory element composed of two adjacent AP-1-like binding sites and is transactivated by the AP-1 complex (Yang et al., 2002). In addition, JunD up-regulates the expression of the human ferritin gene by oxidative stress via an ARE enhancer containing two AP-1 motifs located at $-4.5 \mathrm{~kb}$ upstream region of the human ferritin $\mathrm{H}$ gene (Tsuji, 2005).

The deletion of the region between -1496 to $-480 \mathrm{bp}$ of the 5 'flank of Aldhlal gene relative to the transcription start site completely abolished transactivation by c-Jun. Analysis of this region by Genomatrix software indicates the presence of two AP-1-like 
elements at positions -1069 (site C) and -758 (site B). The sequence of the AP-1-like elements at position -758 (ATGATTCA) and -1069 (GCTCAGTCA) contains a single nucleotide mismatch with the AP-1 consensus TGAG/CTCA found in the human collagenase gene. The difference in the AP-1 motifs between Aldhlal and human collagenase gene could explain the differential regulation of these genes by AP-1 complexes. While the collagenase gene is regulated by c-Jun/c-fos heterodimer, Aldhla1 gene expression is mediated by c-Jun homodimer. The mutation of the AP-1 sequence at position -758 significantly impaired c-Jun-mediated transactivation of Aldh1al reporter activity. However, the activation of Aldh1a1 activity was modestly affected by mutagenesis of the AP-1-like sequence at positions -1069, while mutation of both AP-1 sites completely abolished c-Jun-dependent transactivation. Thus, transient transfection studies indicate that both AP-1-like elements are important for c-Jun-mediated activation of Aldh1a1 reporter activity with the AP-1-like motif at position -758 playing a more critical role.

EMSA and super shift analysis demonstrate the presence of c-Jun, but not c-fos or $\mathrm{Nrf} 2$ in the nuclear protein complex bound to the AP-1 like sequence at -758 , suggesting the importance of AP-1 sequence at -758 in c-Jun-mediated activation of Aldh1a1 gene. However, the AP-1 element at -1069 did not form protein complexes even when incubated with high concentrations of nuclear extracts from HepG2 cells transfected with c-Jun encoding plasmid. This observation is in contrast to the transient transfection studies that showed mutation of site -1069 modestly decreased the activity of Aldh1al promoter by c-Jun. The functional importance of the AP-1 sequence at -758 and -1069 in electrophile-mediated activation of Aldhla1 gene was tested by ChIP experiment. The 
binding of c-Jun to both AP-1 elements at -758 and -1069 in the promoter of Aldh1a1 gene was strongly increased by treatment of mice with BHA. The enhanced recruitment of c-Jun to the AP-1 element at -1069 by BHA is not consistent with the EMSA and transient transfection studies. However, due to the proximity of the two AP-1 sites, which are less than $500 \mathrm{bp}$ apart, it may be difficult to obtain DNA fragments with the separate AP-1 elements by sonication.

In summary, we describe an AP-1 sequence at positions -758 that plays a critical role in Aldhlal gene expression by electrophiles. The deletion and mutagenesis of this AP-1-like sequence significantly abrogate activation of Aldh1a1 reporter activity by cJun. The formation of nuclear protein complex with the AP-1like sequence -758 containing c-Jun was detected by EMSA. BHA promotes binding of c-Jun to the AP-1 responsive sequence at -758 within the Aldh1a1 proximal promoter that regulates gene expression. Transient transfection experiments also demonstrate that the AP-1 like sequence at -1069 plays a modest role in electrophile-induced expression of Aldh1a1 gene. 


\section{CHAPTER V}

\section{DISCUSSION AND CONCLUSIONS}

Aldehydes generated by the process of lipid peroxidation are implicated in the pathogenesis of several oxidative stress-associated pathologies, such as steatohepatitis, liver fibrosis, atherosclerosis, myocardial ischemia-reperfusion injury, cancer and cataractogenesis. Humans have elaborate systems to metabolize these toxic electrophiles and prevent oxidative damage, including the aldehyde dehydrogenases superfamily of enzymes. To date, the relative contribution of the different Aldh isozymes in cellular detoxification of lipid aldehydes especially in mouse liver is highly controversial. Moreover, all previous characterization studies were done using rat cytosol with limited or no studies in mice. Previous studies with human and rat ALDHs demonstrate that ALDH3A1 but not ALDH1A1 provides protection against lipid derived aldehydes (Townsend et al., 2001). However, recent studies with Aldh1a1 ${ }^{-1-}$ mice showed that ALDH1A1 has the potential to protect against these toxic species (Choudhary et al., 2005; Lassen et al., 2007). In chapter II, we presented ample evidence that Aldh1a1 plays a major role in cellular defense against oxidative damage induced by reactive lipid aldehydes in mouse liver. Aldh1a1 exhibits far higher affinity and catalytic efficiencies for lipid aldehydes and GS-conjugates compared to Aldh2 or Aldh3a1. The endogenous Aldhlal gene was highly expressed in mouse liver and liver-derived cell lines compared to Aldh2 and Aldh3a1. The knockdown of Aldh1a1 expression by siRNA caused 
Hepa-1c1c7 cells to be more sensitive to acrolein-induced cell death and resulted in increased accumulation of acrolein-protein adducts and caspase 3 activation. Moreover, previous studies demonstrate high levels of Aldhlal gene expression in the liver and lens, two organs noted for generation of ROS and cytotoxic lipid aldehydes, suggest the importance of Aldh1a1 in cellular protection against oxidative stress-induced damage. These results indicate that mechanisms to induce the expression of Aldhlal gene in mouse liver may be a useful strategy to prevent inflammatory diseases, such as the progression of hepatic steatosis to steatohepatitis.

Microarray analysis of gene expression profiling in mouse liver by electrophiles such as BHA or acrolein demonstrates the specific up-regulation of several antioxidant and electrophile detoxification genes, including Aldh1a1. QRT-PCR analysis confirmed the activation of Aldh1al and other electrophile detoxification genes such as Nqo-1, Ho1 and Gstm1 in mice liver by acrolein or BHA. These results are consistent with previous studies that indicate that Aldhlal gene expression is induced by a variety of microsomal enzyme inducers such as phenobarbital (PB) and BHA (Alnouti and Klaassen, 2008). Consistent with the mRNA expression, the cytosolic Aldh1a1 activity was significantly induced in mice liver treated with BHA or acrolein but not by SFN.

The signaling pathway involved in electrophile-mediated activation of Aldhlal gene in mouse liver is unknown. One mechanism underlying electrophile-mediated induction of Aldh1a1 gene expression can include electrophilic adduction to signaling proteins involved in Aldh1a1 gene regulation. Electrophiles binding to keap1 promote Nrf2 nuclear localization and transactivation of Nrf2-responsive genes. However, while BHA-induced expression of Nqo1, Ho-1 and Gstm1 genes in WT mice were significantly 
reduced in $\mathrm{Nrf}^{-/-}$mice, Aldhlal gene was still responsive to $\mathrm{BHA}$ in $\mathrm{Nrf}^{-/-}$mice liver indicating that BHA-mediated activation of Aldh1al gene is independent of $\mathrm{Nrf} 2$. This result is consistent with previous studies using Keap-1 knockdown (keap1-kd) mice with constitutively active Nrf2 (Reisman et al., 2009). The mRNA expression of Nqo1 and Gstm1 genes were significantly increased in keap1-kd mice compared to WT control, suggesting that the inducible expression of these genes is Nrf2 dependent. However, Aldh1a1 mRNA expression in keap1-kd mice liver was comparable to that of WT mice, confirming that electrophile-induced expression of Aldh1a1 gene is regulated by Nrf2independent mechanism. Even though the 5' flanking sequence of Aldh1a1 gene contains numerous Nrf2-like binding sites (ARE), Nrf2 failed to stimulate the activity of Aldh1a1luciferase reporter, which further supports our hypothesis that electrophile-induced expression of Aldh1a1 gene is independent of Nrf2.

We hypothesized that electrophile-induced transcription of Aldhlal gene is mediated by activation of AP-1 gene. Previous studies demonstrate that the phenolic electrophilic compounds and acrolein induce the expression of cytoprotective genes by stimulating the expression and activity of AP-1 proteins. The activation of c-Jun/AP-1 by oxidative stress during acute hepatitis served a hepatoprotective function by transcriptional up-regulation of Nos 2 gene (Hasselblatt et al., 2007). JunD was recently shown to regulate the human ferritin $\mathrm{H}$ gene expression involved in antioxidant defense and protection against oxidative stress-mediated cytotoxicity (Tsuji, 2005). We showed that the c-jun and c-fos mRNA expression were significantly increased by BHA or acrolein in the livers of WT mice and Hepalc1c7, which correlate with enhanced Aldh1a1 mRNA expression. Furthermore, over-expression of c-Jun, JunD or JunB 
induced endogenous Aldh1a1 protein expression in mouse liver-derived cell lines. The low basal expression of Aldhla1 gene expression in $\mathrm{Nrf}^{-/-}$mice could possibly be attributed to reduced expression of c-Jun and c-fos gene in these mice. The transactivation potential of c-Jun is enhanced by JNK mediated phosphorylation. BHA and acrolein augments the protein levels and activity of c-Jun compared to control. Moreover, the phosphorylation of c-Jun by acrolein correlates with the activation of its upstream kinase, JNK. These results established that electrophile-induced transcription of Aldh1a1 gene is mediated by enhanced expression and activity of AP-1 genes. Transient transfection analysis with c-Jun dominant negative proteins (TAM67) further confirmed that activation of Aldh1al-luciferase reporter activity by acrolein is dependent on cJun/AP-1.

Lipid aldehydes contribute to the up-regulation of electrophile detoxifying genes by modulating the activity of protein kinases, such as JNK, p38 and ERK (Wu et al., 2006;Zhang and Forman, 2008). Experiments using specific inhibitors of JNK, ERK or MEK1 revealed that the treatment of HepG2 with JNK (SP600125) or ERK/MEK1 (PD98059) inhibitors block acrolein or c-Jun-mediated transactivation of Aldh1a1 promoter activity. However, the phosphatase inhibitor (okadaic acid) significantly increased Aldh1a1 promoter activity but had no effect on c-Jun-induced Aldh1a1 luciferase activity. These results suggest that MAPK, such as JNK is essential for transactivation of Aldhlal gene in HepG2 cells.

The AP-1 proteins regulate gene expression as either homodimers of jun or heterodimers with fos family. Transient transfection studies indicate that all the jun family proteins (c-Jun, Jun D and Jun B) activate Aldh1a1-luciferase reporter activity. 
We also noticed that while Fral and Fral had no effect on c-Jun-mediated transactivation of Aldh1a1-luciferase activity, over-expression of c-Fos inhibited activation of Aldh1a1 reporter activity by c-Jun. Abrogation of c-Jun-dependent gene activation by c-Fos was previously reported by Marden et al., who demonstrated that whereas c-Jun homodimers strongly activated CYP2J2 expression, heterodimers formed between c-Fos and c-Jun were inactive (Marden et al., 2003). Thus, mouse Aldhlal and human CYP2J2 genes show similar pattern of regulation by AP-1 with the predominant AP- 1 complex that causes enhanced Aldhla1 promoter luciferase activity being c-Jun homodimer and not cJun/c-Fos heterodimer.

Previous studies demonstrate that Nrf2 acts as a binding partner for c-Jun in transcriptional activation of cytoprotective gene. For example, the activation of CYP2J2 gene expression by BHA dependent on functional interaction between c-Jun and Nrf2 (Lee and Murray, 2010). However, Nrf2 had no effect on c-Jun-mediated transactivation of Aldhlal-luciferase activity. Electrophiles, such as acrolein and HNE have been reported to enhance the expression of cytoprotective genes by activation of nuclear factor $\kappa \mathrm{B}(\mathrm{NF}-\kappa \mathrm{B})$ transcription factor. TNF $\alpha$ is a pro-inflammatory cytokines stimulated by cytotoxic aldehydes and a known activator of the NF- $\mathrm{kB}$ signaling pathway. While TNF $\alpha$ treatments did not activate Aldhlal-luciferase activity, it inhibited c-Jun-mediated activation of Aldhla1-luciferase activity, suggesting that NF- $\mathrm{kB}$ activation abrogates cJun-induced activation of Aldhlal activity. This is consistent with in vivo results in mouse liver which demonstrate that activators of NF- $\mathrm{kB}$ signaling pathway such as high fat diet causes significant reduction in Aldhlal gene expression. 
The mechanism by which AP-1 proteins modulate Aldh1a1 gene expression by electrophiles is still unknown. Previous studies demonstrate that the up-regulation of CYP2J2 gene transcription by BHA was dependent on the binding of c-Jun and Nrf2 to an atypical AP-1-like element in the proximal promoter of CYP2J2 (Lee and Murray, 2010). Analysis of the 5'-flanking sequence of Aldh1al gene indicates the presence of four AP-1-like elements. The promoter deletion and mutagenesis studies indicate that two AP-1-like elements between -1496 to -480 bp of the 5'-flank of Aldh1a1 gene relative to the transcription start site are important for c-Jun-mediated activation of Aldh1a1 reporter activity with the AP-1-like motif at position -758 playing a more critical role.

EMSA and super shift analysis demonstrate the presence of $\mathrm{c}$-Jun, but not $\mathrm{c}$-fos or Nrf2 in the nuclear protein complex bound to the AP-1 like sequence at -758, suggesting the importance of AP-1 sequence at -758 in c-Jun-mediated activation of Aldh1al gene. The AP-1 element at -1069 apparently did not form protein complexes with extracts from HepG2. This observation contrasts our mutagenesis studies which demonstrate that the 1069 site modestly affect c-Jun-mediated transactivation of Aldhlal promoter. The in vivo binding of c-Jun to both AP-1 sequences at -758 and -1069 was strongly increased by treatment of mice with BHA detected by ChIP experiment. However, the enhanced recruitment of c-Jun to the -1069 site by BHA might be due to close proximity ( $<500 \mathrm{bp})$ between this site and the -758 site, it may be difficult to obtain DNA fragments with the separate AP-1 elements by sonication. Future ChIP experiment where the chromatin is fragmented by enzymatic digestion using micrococcal nuclease will allow one to examine whether BHA promotes binding of c-Jun to both AP-1 sites. 
In conclusion, we showed that Aldh1a1 has both electrophile detoxification and antiapoptotic roles in mouse liver and liver-derived cell lines. We also have provided evidence that Aldh1a1 gene can be induced by electrophiles such as acrolein and BHA and that increased expression of Aldhlal gene by electrophiles was dependent on cJun/AP-1 and not Nrf2 or NF-kB. Aldh1al gene expression was modulated by c-Jun homodimer and not c-Jun/c-fos heterodimer. The MAPK especially JNK also plays a crucial role in electrophile mediated activation of Aldh1a1 gene. The deletion and mutagenesis of two AP-1-like sequences at positions -758 and -1069 significantly abrogate activation of Aldhlal reporter activity by c-Jun. The formation of nuclear protein complex with the AP-1like sequence -758 containing c-Jun was detected by EMSA. BHA promotes binding of c-Jun to the AP-1 responsive sequence at -758 within the Aldh1a1 proximal promoter that regulates gene expression. Due to the lipid aldehyde detoxification and anti-apoptotic role of Aldh1al in mice liver, an understanding of the mechanism to activate Aldhlal gene expression might provide a useful rationale for therapeutic protection and amelioration of oxidative stress-induced pathologies. The proposed model for the activation of Aldh1a1 transcription by electrophiles is illustrated in Figure 33. 


\section{BHA, Acrolein}

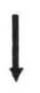

\section{Oxidative stress}

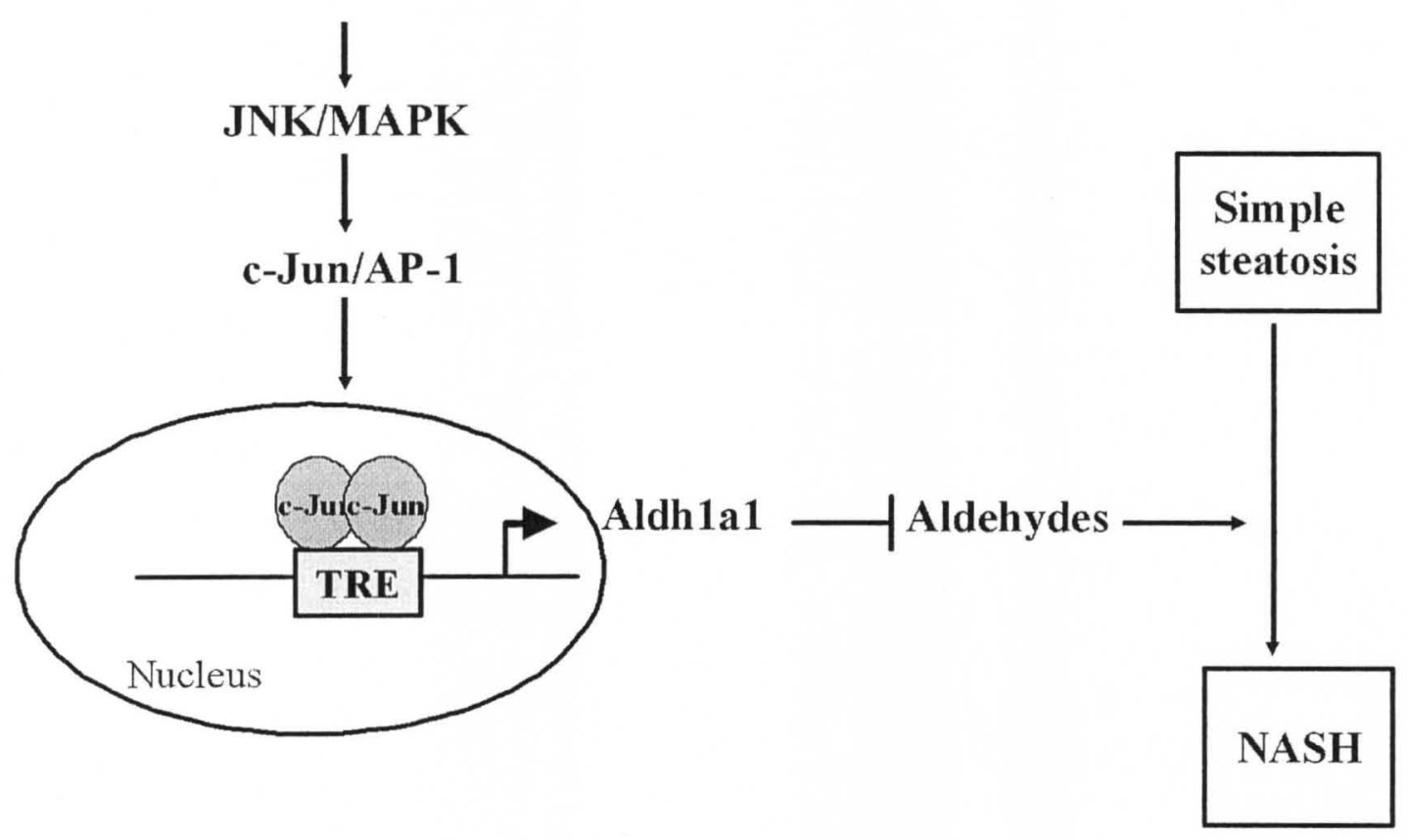

Figure 33. Proposed schematic model for the activation of Aldh1a1 gene by electrophiles.

Electrophiles such as BHA and acrolein are known to induce oxidative stress in cells by redox cycling and depletion of cellular reduced GSH, respectively. In response to oxidative and electrophilic stress, stress-activated protein kinase such as JNK phosphorylates c-Jun/AP-1 and enhances its transactivating potential. There is enhanced recruitment of c-Jun homodimer to AP-1-like element in the proximal promoter of Aldh1a1 gene resulting in transcriptional activation of Aldh1a1 gene. Increased expression of Aldh1a1 leads to enhanced metabolism and detoxification of acrolein and HNE, thereby inhibiting the progression of fatty liver to steatohepatitis (NASH). 


\section{FUTURE STUDIES.}

Non-alcoholic fatty liver disease (NAFLD) is a spectrum of pathologies ranging from simple triglyceride accumulation in hepatocytes (simple steatosis) to steatosis with inflammation (steatohepatitis, NASH), fibrosis, cirrhosis and hepatocellular carcinoma (Browning and Horton, 2004). Hepatic steatosis is benign and reversible. However, formation of NASH may progress to fibrosis and other end-stage liver diseases. The prevalence of NAFLD in the general population is estimated to be between $14-24 \%$ with $\approx 30$ million Americans affected with the disease. Cirrhosis of the liver accounts for $14 \%$ of liver transplants in the US. The "two hit model" was proposed to explain the etiology of NAFLD (Day and James, 1998). The "first hit" is attributed to insulin resistance, which leads to simple steatosis. The "second hit" is due to oxidative stress, which might be a consequence of increased production of ROS in the liver, relative to antioxidants, generated from activation of alternative pathways for fatty acid oxidation such as the peroxisomal $\beta$ oxidation and microsomal $\omega$ oxidation catalyzed primarily by acyl-CoA oxidase and cytochrome P450 4A, respectively. ROS are relatively short-lived and exert local effects. However, they initiate lipid peroxidation in cells by attacking PUFA, which results in formation of aldehyde products such as acrolein, HNE and MDA (Esterbauer et al., 1991). These aldehydes have longer half-lives than ROS and can diffuse to distant sites of their production, thereby amplifying the effects of oxidative ROS. It is proposed that the deleterious effect of ROS is mediated by these aldehydes (Esterbauer et al., 1991;Esterbauer, 1993).

Reactive aldehydes are implicated in the initiation and perpetuation of inflammation in NASH. The accumulation of reactive aldehydes in the liver can promote 
the influx of inflammatory cells by stimulating the production of the proinflammatory cytokines, TNF $\alpha$, thereby amplifying inflammatory response (Browning and Horton, 2004; Esterbauer et al., 1991). In addition, reactive aldehydes are also known to activate hepatic stellate cells leading to collagen deposition (Browning and Horton, 2004). Thus, lipid aldehydes can promote progression from steatosis to NASH by directly inducing hepatocyte death and necrosis, inflammation and liver fibrosis. We showed that Aldhla1 is a major enzyme in mouse liver involved in oxidative metabolism and detoxification of lipid aldehydes. Thus, we hypothesize that activation of Aldh1a1 gene expression in the liver is a therapeutic strategy to prevent progression to NASH. Future experiment should examine whether Aldh1 $11^{-/ .}$mice are more susceptible to NASH than WT mice on long term high fat diet (HFD), a standard nutritional method for stimulating NAFLD and NASH in rodents (Kim et al., 2004). Male C57BL/6 (WT) mice and Aldhla1 ${ }^{-1-}$ mice on C57BL/6 background could be placed on low fat diet (LFD) or HFD for 12 weeks. Subsequently, the liver can be examined for lipid accumulations using $\mathrm{H}$ and $\mathrm{E}$ staining. Markers of oxidative stress can be examined, such as total, reduced (GSH) and oxidized (GSSG) glutathione, and aldehyde accumulations in the liver. Finally, the levels of proinflammatory cytokines, such as TNF $\alpha$, IL- $1 \beta$ and Cox 2 can be compared in both genotypes. Aldhla1 gene expression is induced in mice liver by phenobarbital (PB) (Alnouti and Klaassen, 2008). Future studies could also assess whether activation of Aldh1a1 gene expression in the liver by $\mathrm{PB}$ can prevent NASH. C57BL/6 mice can be administered either saline or $100 \mathrm{mg} / \mathrm{kg}$ PB (i.p) in saline daily for 7 days. The markers of oxidative stress and aldehyde accumulation, pro-inflammatory cytokines and lipid accumulations can be assessed in control and treated group of mice. 


\section{REFERENCES}

Abiko Y, Miura T, Phuc B H, Shinkai Y and Kumagai Y (2011) Participation of Covalent Modification of Keap1 in the Activation of Nrf2 by Tert-Butylbenzoquinone, an Electrophilic Metabolite of Butylated Hydroxyanisole. Toxicol Appl Pharmacol.

Alnouti Y and Klaassen C D (2008) Tissue Distribution, Ontogeny, and Regulation of Aldehyde Dehydrogenase (Aldh) Enzymes MRNA by Prototypical Microsomal Enzyme Inducers in Mice. Toxicol Sci 101:51-64.

Amunom I, Stephens L J, Tamasi V, Cai J, Pierce W M, Jr., Conklin D J, Bhatnagar A, Srivastava S, Martin M V, Guengerich F P and Prough R A (2007) Cytochromes P450 Catalyze Oxidation of Alpha,Beta-Unsaturated Aldehydes. Arch Biochem Biophys 464:187-196.

Andreola F, Fernandez-Salguero P M, Chiantore M V, Petkovich M P, Gonzalez F J and De Luca L M (1997) Aryl Hydrocarbon Receptor Knockout Mice (AHR-/-) Exhibit Liver Retinoid Accumulation and Reduced Retinoic Acid Metabolism. Cancer Res 57:28352838 .

Beauchamp RO, Jr., Andjelkovich D A, Kligerman A D, Morgan K T and Heck H D (1985) A Critical Review of the Literature on Acrolein Toxicity. Crit Rev Toxicol 14:309-380.

Beretta M, Gruber K, Kollau A, Russwurm M, Koesling D, Goessler W, Keung W M, Schmidt K and Mayer B (2008) Bioactivation of Nitroglycerin by Purified Mitochondrial and Cytosolic Aldehyde Dehydrogenases. J Biol Chem 283:17873-17880.

Berhane K and Mannervik B (1990) Inactivation of the Genotoxic Aldehyde Acrolein by Human Glutathione Transferases of Classes Alpha, Mu, and Pi. Mol Pharmacol 37:251254.

Browning JD and Horton J D (2004) Molecular Mediators of Hepatic Steatosis and Liver Injury. $J$ Clin Invest 114:147-152.

Burcham PC and Fontaine F (2001) Extensive Protein Carbonylation Precedes AcroleinMediated Cell Death in Mouse Hepatocytes. J Biochem Mol Toxicol 15:309-316.

Chen CH, Budas G R, Churchill E N, Disatnik M H, Hurley T D and Mochly-Rosen D (2008) Activation of Aldehyde Dehydrogenase-2 Reduces Ischemic Damage to the Heart. Science 321:1493-1495. 
Chen J, Henderson G I and Freeman G L (2001) Role of 4-Hydroxynonenal in Modification of Cytochrome c Oxidase in Ischemia/Reperfused Rat Heart. J Mol Cell Cardiol 33:1919-1927.

Chen JJ, Schenker S and Henderson G I (1997) 4-Hydroxynonenal Levels Are Enhanced in Fetal Liver Mitochondria by in Utero Ethanol Exposure. Hepatology 25:142-147.

Cheng JZ, Singhal S S, Sharma A, Saini M, Yang Y, Awasthi S, Zimniak P and Awasthi Y C (2001) Transfection of MGSTA4 in HL-60 Cells Protects Against 4Hydroxynonenal-Induced Apoptosis by Inhibiting JNK-Mediated Signaling. Arch Biochem Biophys 392:197-207.

Choi HS and Moore D D (1993) Induction of C-Fos and C-Jun Gene Expression by Phenolic Antioxidants. Mol Endocrinol 7:1596-1602.

Choudhary S, Srivastava S, Xiao T, Andley U P, Srivastava S K and Ansari N H (2003) Metabolism of Lipid Derived Aldehyde, 4-Hydroxynonenal in Human Lens Epithelial Cells and Rat Lens. Invest Ophthalmol Vis Sci 44:2675-2682.

Choudhary S, Xiao T, Vergara L A, Srivastava S, Nees D, Piatigorsky J and Ansari N H (2005) Role of Aldehyde Dehydrogenase Isozymes in the Defense of Rat Lens and Human Lens Epithelial Cells Against Oxidative Stress. Invest Ophthalmol Vis Sci 46:259-267.

Choudhary S, Zhang W, Zhou F, Campbell G A, Chan L L, Thompson E B and Ansari N H (2002) Cellular Lipid Peroxidation End-Products Induce Apoptosis in Human Lens Epithelial Cells. Free Radic Biol Med 32:360-369.

Chute JP, Muramoto G G, Whitesides J, Colvin M, Safi R, Chao N J and McDonnell D P (2006) Inhibition of Aldehyde Dehydrogenase and Retinoid Signaling Induces the Expansion of Human Hematopoietic Stem Cells. Proc Natl Acad Sci US A 103:1170711712.

Collard F, Vertommen D, Fortpied J, Duester G and Van S E (2007) Identification of 3Deoxyglucosone Dehydrogenase As Aldehyde Dehydrogenase 1A1 (Retinaldehyde Dehydrogenase 1). Biochimie 89:369-373.

Conklin D, Prough R and Bhatanagar A (2007) Aldehyde Metabolism in the Cardiovascular System. Mol Biosyst 3:136-150.

Conklin DJ, Barski O A, Lesgards J F, Juvan P, Rezen T, Rozman D, Prough R A, Vladykovskaya E, Liu S, Srivastava S and Bhatnagar A (2010) Acrolein Consumption Induces Systemic Dyslipidemia and Lipoprotein Modification. Toxicol Appl Pharmacol 243:1-12.

Day CP and James O F (1998) Steatohepatitis: a Tale of Two "Hits"? Gastroenterology 114:842-845. 
Deitrich RA, Bludeau P, Stock T and Roper M (1977) Induction of Different Rat Liver Supernatant Aldehyde Dehydrogenases by Phenobarbital and Tetrachlorodibenzo-pDioxin. J Biol Chem 252:6169-6176.

Dwivedi S, Sharma R, Sharma A, Zimniak P, Ceci J D, Awasthi Y C and Boor P J (2006) The Course of CCl4 Induced Hepatotoxicity Is Altered in MGSTA4-4 Null (-/-) Mice. Toxicology 218:58-66.

Eaton P, Li J M, Hearse D J and Shattock M J (1999) Formation of 4-Hydroxy-2Nonenal-Modified Proteins in Ischemic Rat Heart. Am J Physiol 276:H935-H943.

Eferl R and Wagner E F (2003) AP-1: a Double-Edged Sword in Tumorigenesis. Nat Rev Cancer 3:859-868.

Elizondo G, Corchero J, Sterneck E and Gonzalez F J (2000) Feedback Inhibition of the Retinaldehyde Dehydrogenase Gene ALDH1 by Retinoic Acid Through Retinoic Acid Receptor Alpha and CCAAT/Enhancer-Binding Protein Beta. J Biol Chem 275:3974739753.

Elizondo G, Medina-Diaz I M, Cruz R, Gonzalez F J and Vega L (2009) Retinoic Acid Modulates Retinaldehyde Dehydrogenase 1 Gene Expression Through the Induction of GADD153-C/EBPbeta Interaction. Biochem Pharmacol 77:248-257.

Ellis EM (2007) Reactive Carbonyls and Oxidative Stress: Potential for Therapeutic Intervention. Pharmacol Ther 115:13-24.

Esterbauer H (1993) Cytotoxicity and Genotoxicity of Lipid-Oxidation Products. Am J Clin Nutr 57:779S-785S.

Esterbauer H, Schaur R J and Zollner H (1991) Chemistry and Biochemistry of 4Hydroxynonenal, Malonaldehyde and Related Aldehydes. Free Radic Biol Med 11:81128.

Feron VJ, Til H P, de V F, Woutersen R A, Cassee F R and van Bladeren P J (1991) Aldehydes: Occurrence, Carcinogenic Potential, Mechanism of Action and Risk Assessment. Mutat Res 259:363-385.

Goedde HW, Agarwal D P, Fritze G, Meier-Tackmann D, Singh S, Beckmann G, Bhatia K, Chen L Z, Fang B, Lisker R and . (1992) Distribution of ADH2 and ALDH2 Genotypes in Different Populations. Hum Genet 88:344-346.

Guimond J, Devost D, Brodeur H, Mader S and Bhat P V (2002) Characterization of the Rat RALDH1 Promoter. A Functional CCAAT and Octamer Motif Are Critical for Basal Promoter Activity. Biochim Biophys Acta 1579:81-91.

Gyamfi MA, Kocsis M G, He L, Dai G, Mendy A J and Wan Y J (2006) The Role of Retinoid X Receptor Alpha in Regulating Alcohol Metabolism. J Pharmacol Exp Ther 319:360-368. 
Hammer A, Ferro M, Tillian H M, Tatzber F, Zollner H, Schauenstein E and Schaur R J (1997) Effect of Oxidative Stress by Iron on 4-Hydroxynonenal Formation and Proliferative Activity in Hepatomas of Different Degrees of Differentiation. Free Radic Biol Med 23:26-33.

Hartley DP, Ruth J A and Petersen D R (1995) The Hepatocellular Metabolism of 4Hydroxynonenal by Alcohol Dehydrogenase, Aldehyde Dehydrogenase, and Glutathione S-Transferase. Arch Biochem Biophys 316:197-205.

Hasselblatt P, Rath M, Komnenovic V, Zatloukal K and Wagner E F (2007) Hepatocyte Survival in Acute Hepatitis Is Due to C-Jun/AP-1-Dependent Expression of Inducible Nitric Oxide Synthase. Proc Natl Acad Sci US A 104:17105-17110.

Hsu LC, Chang W C, Hoffmann I and Duester G (1999) Molecular Analysis of Two Closely Related Mouse Aldehyde Dehydrogenase Genes: Identification of a Role for Aldh1, but Not Aldh-Pb, in the Biosynthesis of Retinoic Acid. Biochem J 339 ( Pt 2):387-395.

Hsu LC, Chang W C and Yoshida A (2000) Mouse Type-2 Retinaldehyde Dehydrogenase (RALDH2): Genomic Organization, Tissue-Dependent Expression, Chromosome Assignment and Comparison to Other Types. Biochim Biophys Acta 1492:289-293.

Hu R, Xu C, Shen G, Jain M R, Khor T O, Gopalkrishnan A, Lin W, Reddy B, Chan J Y and Kong A N (2006) Identification of Nrf2-Regulated Genes Induced by Chemopreventive Isothiocyanate PEITC by Oligonucleotide Microarray. Life Sci 79:1944-1955.

Huang HC, Nguyen T and Pickett C B (2000) Regulation of the Antioxidant Response Element by Protein Kinase C-Mediated Phosphorylation of NF-E2-Related Factor 2. Proc Natl Acad Sci US A 97:12475-12480.

Huang HC, Nguyen T and Pickett C B (2002) Phosphorylation of Nrf2 at Ser-40 by Protein Kinase C Regulates Antioxidant Response Element-Mediated Transcription. $J$ Biol Chem 277:42769-42774.

Iles KE and Liu R M (2005) Mechanisms of Glutamate Cysteine Ligase (GCL) Induction by 4-Hydroxynonenal. Free Radic Biol Med 38:547-556.

Karin M (1999) The Beginning of the End: IkappaB Kinase (IKK) and NF-KappaB Activation. J Biol Chem 274:27339-27342.

Kim S, Sohn I, Ahn J I, Lee K H, Lee Y S and Lee Y S (2004) Hepatic Gene Expression Profiles in a Long-Term High-Fat Diet-Induced Obesity Mouse Model. Gene 340:99-109.

Klyosov AA, Rashkovetsky L G, Tahir M K and Keung W M (1996) Possible Role of Liver Cytosolic and Mitochondrial Aldehyde Dehydrogenases in Acetaldehyde Metabolism. Biochemistry 35:4445-4456. 
Kovacic-Milivojevic B and Gardner D G (1992) Divergent Regulation of the Human Atrial Natriuretic Peptide Gene by C-Jun and C-Fos. Mol Cell Biol 12:292-301.

Kuo CL, Vaz A D and Coon M J (1997) Metabolic Activation of Trans-4-Hydroxy-2Nonenal, a Toxic Product of Membrane Lipid Peroxidation and Inhibitor of P450 Cytochromes. J Biol Chem 272:22611-22616.

Kwak MK, Kensler T W and Casero R A, Jr. (2003) Induction of Phase 2 Enzymes by Serum Oxidized Polyamines Through Activation of Nrf2: Effect of the Polyamine Metabolite Acrolein. Biochem Biophys Res Commun 305:662-670.

Lamb JA, Ventura J J, Hess P, Flavell R A and Davis R J (2003) JunD Mediates Survival Signaling by the JNK Signal Transduction Pathway. Mol Cell 11:1479-1489.

Lamle J, Marhenke S, Borlak J, von W R, Eriksson C J, Geffers R, Manns M P, Yamamoto M and Vogel A (2008) Nuclear Factor-Eythroid 2-Related Factor 2 Prevents Alcohol-Induced Fulminant Liver Injury. Gastroenterology 134:1159-1168.

Lassen N, Bateman J B, Estey T, Kuszak J R, Nees D W, Piatigorsky J, Duester G, Day B J, Huang J, Hines L M and Vasiliou V (2007) Multiple and Additive Functions of ALDH3A1 and ALDH1A1: Cataract Phenotype and Ocular Oxidative Damage in Aldh3a1(-/-)/Aldh1a1(-/-) Knock-Out Mice. J Biol Chem 282:25668-25676.

Lee AC and Murray M (2010) Up-Regulation of Human CYP2J2 in HepG2 Cells by Butylated Hydroxyanisole Is Mediated by C-Jun and Nrf2. Mol Pharmacol 77:987-994.

Lee JM, Calkins M J, Chan K, Kan Y W and Johnson J A (2003) Identification of the NF-E2-Related Factor-2-Dependent Genes Conferring Protection Against Oxidative Stress in Primary Cortical Astrocytes Using Oligonucleotide Microarray Analysis. J Biol Chem 278:12029-12038.

Leonard MO, Kieran N E, Howell K, Burne M J, Varadarajan R, Dhakshinamoorthy S, Porter A G, O'Farrelly C, Rabb H and Taylor C T (2006) Reoxygenation-Specific Activation of the Antioxidant Transcription Factor Nrf2 Mediates Cytoprotective Gene Expression in Ischemia-Reperfusion Injury. FASEB $J$ 20:2624-2626.

Li J, Yao S and Zhang Y (2005) The Role of C-Jun in the AP-1 Activation Induced by Naturally Occurring Isothiocyanates. Food Chem Toxicol 43:1373-1380.

Lindahl R and Petersen D R (1991) Lipid Aldehyde Oxidation As a Physiological Role for Class 3 Aldehyde Dehydrogenases. Biochem Pharmacol 41:1583-1587.

Liu XY, Yang Z H, Pan X J, Zhu M X and Xie J P (2010) Gene Expression Profile and Cytotoxicity of Human Bronchial Epithelial Cells Exposed to Crotonaldehyde. Toxicol Lett 197:113-122. 
Makia NL, Bojang P, Falkner K C, Conklin D J and Prough R A (2011) Murine Hepatic Aldehyde Dehydrogenase 1a1 Is a Major Contributor to Oxidation of Aldehydes Formed by Lipid Peroxidation. Chem Biol Interact 191:278-287.

Marchitti SA, Brocker C, Stagos D and Vasiliou V (2008) Non-P450 Aldehyde Oxidizing Enzymes: the Aldehyde Dehydrogenase Superfamily. Expert Opin Drug Metab Toxicol 4:697-720.

Marden JJ, Zhang Y, Oakley F D, Zhou W, Luo M, Jia H P, McCray P B, Jr., Yaniv M, Weitzman J B and Engelhardt J F (2008) JunD Protects the Liver From Ischemia/Reperfusion Injury by Dampening AP-1 Transcriptional Activation. $J$ Biol Chem 283:6687-6695.

Marden NY, Fiala-Beer E, Xiang S H and Murray M (2003) Role of Activator Protein-1 in the Down-Regulation of the Human CYP2J2 Gene in Hypoxia. Biochem J 373:669680.

Moreb JS, Mohuczy D, Ostmark B and Zucali J R (2007) RNAi-Mediated Knockdown of Aldehyde Dehydrogenase Class-1A1 and Class-3A1 Is Specific and Reveals That Each Contributes Equally to the Resistance Against 4-Hydroperoxycyclophosphamide. Cancer Chemother Pharmacol 59:127-136.

Nakamura Y, Kumagai T, Yoshida C, Naito Y, Miyamoto M, Ohigashi H, Osawa T and Uchida K (2003) Pivotal Role of Electrophilicity in Glutathione S-Transferase Induction by Tert-Butylhydroquinone. Biochemistry 42:4300-4309.

Nanji AA, Zhao S, Sadrzadeh S M, Dannenberg A J, Tahan S R and Waxman D J (1994) Markedly Enhanced Cytochrome P450 2E1 Induction and Lipid Peroxidation Is Associated With Severe Liver Injury in Fish Oil-Ethanol-Fed Rats. Alcohol Clin Exp Res 18:1280-1285.

Nguyen T, Nioi P and Pickett C B (2009) The Nrf2-Antioxidant Response Element Signaling Pathway and Its Activation by Oxidative Stress. J Biol Chem 284:1329113295.

Nguyen T, Sherratt P J and Pickett C B (2003) Regulatory Mechanisms Controlling Gene Expression Mediated by the Antioxidant Response Element. Annu Rev Pharmacol Toxicol 43:233-260.

Nguyen T, Yang C S and Pickett C B (2004) The Pathways and Molecular Mechanisms Regulating Nrf2 Activation in Response to Chemical Stress. Free Radic Biol Med 37:433-441.

Pappas P, Stephanou P, Karamanakos P, Vasiliou V and Marselos M (2001) Phenobarbital Inducibility and Differences in Protein Expression of an Animal Model. Chem Biol Interact 130-132:275-283. 
Park YS, Misonou Y, Fujiwara N, Takahashi M, Miyamoto Y, Koh Y H, Suzuki K and Taniguchi N (2005) Induction of Thioredoxin Reductase As an Adaptive Response to Acrolein in Human Umbilical Vein Endothelial Cells. Biochem Biophys Res Commun 327:1058-1065.

Pinkus R, Weiner L M and Daniel V (1996) Role of Oxidants and Antioxidants in the Induction of AP-1, NF-KappaB, and Glutathione S-Transferase Gene Expression. $J$ Biol Chem 271:13422-13429.

Pugazhenthi S, Phansalkar K, Audesirk G, West A and Cabell L (2006) Differential Regulation of C-Jun and CREB by Acrolein and 4-Hydroxynonenal. Free Radic Biol Med 40:21-34.

Rahmani M, Peron P, Weitzman J, Bakiri L, Lardeux B and Bernuau D (2001) Functional Cooperation Between JunD and NF-KappaB in Rat Hepatocytes. Oncogene 20:51325142.

Ramu K, Fraiser L H, Mamiya B, Ahmed T and Kehrer J P (1995) Acrolein Mercapturates: Synthesis, Characterization, and Assessment of Their Role in the Bladder Toxicity of Cyclophosphamide. Chem Res Toxicol 8:515-524.

Ranganna K, Yousefipour Z, Nasif R, Yatsu F M, Milton S G and Hayes B E (2002) Acrolein Activates Mitogen-Activated Protein Kinase Signal Transduction Pathways in Rat Vascular Smooth Muscle Cells. Mol Cell Biochem 240:83-98.

Reddy NM, Kleeberger S R, Yamamoto M, Kensler T W, Scollick C, Biswal S and Reddy S P (2007) Genetic Dissection of the Nrf2-Dependent Redox Signaling-Regulated Transcriptional Programs of Cell Proliferation and Cytoprotection. Physiol Genomics 32:74-81.

Reisman SA, Yeager R L, Yamamoto M and Klaassen C D (2009) Increased Nrf2 Activation in Livers From Keap1-Knockdown Mice Increases Expression of Cytoprotective Genes That Detoxify Electrophiles More Than Those That Detoxify Reactive Oxygen Species. Toxicol Sci 108:35-47.

Remmer H, Schenkman J, Estabrook R W, Sasame H, Gillette J, Narasimhulu S, Cooper D Y and Rosenthal O (1966) Drug Interaction With Hepatic Microsomal Cytochrome. Mol Pharmacol 2:187-190.

Rushmore TH, King R G, Paulson K E and Pickett C B (1990) Regulation of Glutathione S-Transferase Ya Subunit Gene Expression: Identification of a Unique XenobioticResponsive Element Controlling Inducible Expression by Planar Aromatic Compounds. Proc Natl Acad Sci US A 87:3826-3830.

Rushmore TH, Morton M R and Pickett C B (1991) The Antioxidant Responsive Element. Activation by Oxidative Stress and Identification of the DNA Consensus Sequence Required for Functional Activity. J Biol Chem 266:11632-11639. 
Rushmore TH and Pickett C B (1991) Xenobiotic Responsive Elements Controlling Inducible Expression by Planar Aromatic Compounds and Phenolic Antioxidants. Methods Enzymol 206:409-420.

Sampey BP, Stewart B J and Petersen D R (2007) Ethanol-Induced Modulation of Hepatocellular Extracellular Signal-Regulated Kinase-1/2 Activity Via 4Hydroxynonenal. J Biol Chem 282:1925-1937.

Sayre LM, Zelasko D A, Harris P L, Perry G, Salomon R G and Smith M A (1997) 4Hydroxynonenal-Derived Advanced Lipid Peroxidation End Products Are Increased in Alzheimer's Disease. J Neurochem 68:2092-2097.

Silva JM and O'Brien P J (1989) Allyl Alcohol- and Acrolein-Induced Toxicity in Isolated Rat Hepatocytes. Arch Biochem Biophys 275:551-558.

Sophos NA and Vasiliou V (2003) Aldehyde Dehydrogenase Gene Superfamily: the 2002 Update. Chem Biol Interact 143-144:5-22.

Srivastata SK, Awasthi S, Wang L, Bhatnagar A, Awasthi Y C and Ansari N H (1996) Attenuation of 4-Hydroxynonenal-Induced Cataractogenesis in Rat Lens by Butylated Hydroxytoluene. Curr Eye Res 15:749-754.

Srivastava S, Chandra A, Wang L F, Seifert W E, Jr., DaGue B B, Ansari N H, Srivastava S K and Bhatnagar A (1998) Metabolism of the Lipid Peroxidation Product, 4Hydroxy-Trans-2-Nonenal, in Isolated Perfused Rat Heart. J Biol Chem 273:1089310900.

Srivastava S, Liu S Q, Conklin D J, Zacarias A, Srivastava S K and Bhatnagar A (2001) Involvement of Aldose Reductase in the Metabolism of Atherogenic Aldehydes. Chem Biol Interact 130-132:563-571.

Stagos D, Chen Y, Brocker C, Donald E, Jackson B C, Orlicky D J, Thompson D C and Vasiliou V (2010) Aldehyde Dehydrogenase 1B1 (ALDH1B1): Molecular Cloning and Characterization of a Novel Mitochondrial Acetaldehyde Metabolizing Enzyme. Drug Metab Dispos.

Stevens JF and Maier C S (2008) Acrolein: Sources, Metabolism, and Biomolecular Interactions Relevant to Human Health and Disease. Mol Nutr Food Res 52:7-25.

Szweda LI, Uchida K, Tsai L and Stadtman E R (1993) Inactivation of Glucose-6Phosphate Dehydrogenase by 4-Hydroxy-2-Nonenal. Selective Modification of an Active-Site Lysine. J Biol Chem 268:3342-3347.

Tanel A and Averill-Bates D A (2007) Inhibition of Acrolein-Induced Apoptosis by the Antioxidant N-Acetylcysteine. J Pharmacol Exp Ther 321:73-83. 
Thimmulappa RK, Mai K H, Srisuma S, Kensler T W, Yamamoto M and Biswal S (2002) Identification of Nrf2-Regulated Genes Induced by the Chemopreventive Agent Sulforaphane by Oligonucleotide Microarray. Cancer Res 62:5196-5203.

Thornberry NA and Lazebnik Y (1998) Caspases: Enemies Within. Science 281:13121316.

Thweatt WD, Harward C N, Sr. and Parrish M E (2007) Measurement of Acrolein and 1,3-Butadiene in a Single Puff of Cigarette Smoke Using Lead-Salt Tunable Diode Laser Infrared Spectroscopy. Spectrochim Acta A Mol Biomol Spectrosc 67:16-24.

Tirumalai R, Rajesh K T, Mai K H and Biswal S (2002) Acrolein Causes Transcriptional Induction of Phase II Genes by Activation of Nrf2 in Human Lung Type II Epithelial (A549) Cells. Toxicol Lett 132:27-36.

Townsend AJ, Leone-Kabler S, Haynes R L, Wu Y, Szweda L and Bunting K D (2001) Selective Protection by Stably Transfected Human ALDH3A1 (but Not Human ALDH1 A1) Against Toxicity of Aliphatic Aldehydes in V79 Cells. Chem Biol Interact 130-132:261-273.

Toyokuni S, Yamada S, Kashima M, Ihara Y, Yamada Y, Tanaka T, Hiai H, Seino Y and Uchida K (2000) Serum 4-Hydroxy-2-Nonenal-Modified Albumin Is Elevated in Patients With Type 2 Diabetes Mellitus. Antioxid Redox Signal 2:681-685.

Tsuji Y (2005) JunD Activates Transcription of the Human Ferritin H Gene Through an Antioxidant Response Element During Oxidative Stress. Oncogene 24:7567-7578.

Tweedie DJ, Fernandez D, Spearman M E, Feldhoff R C and Prough R A (1991) Metabolism of Azoxy Derivatives of Procarbazine by Aldehyde Dehydrogenase and Xanthine Oxidase. Drug Metab Dispos 19:793-803.

Uchida K, Kanematsu M, Morimitsu Y, Osawa T, Noguchi N and Niki E (1998) Acrolein Is a Product of Lipid Peroxidation Reaction. Formation of Free Acrolein and Its Conjugate With Lysine Residues in Oxidized Low Density Lipoproteins. J Biol Chem 273:16058-16066.

Uchida K (2000) Role of Reactive Aldehyde in Cardiovascular Diseases. Free Radic Biol Med 28:1685-1696.

Uchida K, Kanematsu M, Morimitsu Y, Osawa T, Noguchi N and Niki E (1998) Acrolein Is a Product of Lipid Peroxidation Reaction. Formation of Free Acrolein and Its Conjugate With Lysine Residues in Oxidized Low Density Lipoproteins. J Biol Chem 273:16058-16066.

Uchida K and Stadtman E R (1993) Covalent Attachment of 4-Hydroxynonenal to Glyceraldehyde-3-Phosphate Dehydrogenase. A Possible Involvement of Intra- and Intermolecular Cross-Linking Reaction. J Biol Chem 268:6388-6393. 
Uchida K, Toyokuni S, Nishikawa K, Kawakishi S, Oda H, Hiai H and Stadtman E R (1994) Michael Addition-Type 4-Hydroxy-2-Nonenal Adducts in Modified Low-Density Lipoproteins: Markers for Atherosclerosis. Biochemistry 33:12487-12494.

Vasiliou V, Pappa A and Estey T (2004) Role of Human Aldehyde Dehydrogenases in Endobiotic and Xenobiotic Metabolism. Drug Metab Rev 36:279-299.

Wang GW, Guo Y, Vondriska T M, Zhang J, Zhang S, Tsai L L, Zong N C, Bolli R, Bhatnagar A and Prabhu S D (2008) Acrolein Consumption Exacerbates Myocardial Ischemic Injury and Blocks Nitric Oxide-Induced PKCepsilon Signaling and Cardioprotection. J Mol Cell Cardiol 44:1016-1022.

Weitzman JB, Fiette L, Matsuo K and Yaniv M (2000) JunD Protects Cells From P53Dependent Senescence and Apoptosis. Mol Cell 6:1109-1119.

Wu CC, Hsieh C W, Lai P H, Lin J B, Liu Y C and Wung B S (2006) Upregulation of Endothelial Heme Oxygenase-1 Expression Through the Activation of the JNK Pathway by Sublethal Concentrations of Acrolein. Toxicol Appl Pharmacol 214:244-252.

Xiao T, Choudhary S, Zhang W, Ansari N H and Salahudeen A (2003) Possible Involvement of Oxidative Stress in Cisplatin-Induced Apoptosis in LLC-PK1 Cells. $J$ Toxicol Environ Health A 66:469-479.

Xiao T, Shoeb M, Siddiqui M S, Zhang M, Ramana K V, Srivastava S K, Vasiliou V and Ansari N H (2009) Molecular Cloning and Oxidative Modification of Human Lens ALDH1A1: Implication in Impaired Detoxification of Lipid Aldehydes. $J$ Toxicol Environ Health A 72:577-584.

Yang H, Magilnick N, Lee C, Kalmaz D, Ou X, Chan J Y and Lu S C (2005) Nrf1 and Nrf2 Regulate Rat Glutamate-Cysteine Ligase Catalytic Subunit Transcription Indirectly Via NF-KappaB and AP-1. Mol Cell Biol 25:5933-5946.

Yang H, Zeng Y, Lee T D, Yang Y, Ou X, Chen L, Haque M, Rippe R and Lu S C (2002) Role of AP-1 in the Coordinate Induction of Rat Glutamate-Cysteine Ligase and Glutathione Synthetase by Tert-Butylhydroquinone. J Biol Chem 277:35232-35239.

Yu R, Tan T H and Kong A N (1997) Butylated Hydroxyanisole and Its Metabolite TertButylhydroquinone Differentially Regulate Mitogen-Activated Protein Kinases. The Role of Oxidative Stress in the Activation of Mitogen-Activated Protein Kinases by Phenolic Antioxidants. J Biol Chem 272:28962-28970.

Yuan X, Xu C, Pan Z, Keum Y S, Kim J H, Shen G, Yu S, Oo K T, Ma J and Kong A N (2006) Butylated Hydroxyanisole Regulates ARE-Mediated Gene Expression Via Nrf2 Coupled With ERK and JNK Signaling Pathway in HepG2 Cells. Mol Carcinog 45:841850 . 
Zhang $\mathrm{H}$ and Forman $\mathrm{H} \mathrm{J}$ (2008) Acrolein Induces Heme Oxygenase-1 Through PKCDelta and PI3K in Human Bronchial Epithelial Cells. Am J Respir Cell Mol Biol 38:483490.

Zhang M, Shoeb M, Goswamy J, Liu P, Xiao T L, Hogan D, Campbell G A and Ansari N H (2010) Overexpression of Aldehyde Dehydrogenase 1A1 Reduces Oxidation-Induced Toxicity in SH-SY5Y Neuroblastoma Cells. J Neurosci Res 88:686-694.

Zhong S, Machida K, Tsukamoto H and Johnson D L (2011) Alcohol Induces RNA Polymerase III-Dependent Transcription Through C-Jun by Co-Regulating TATABinding Protein (TBP) and Brfl Expression. J Biol Chem 286:2393-2401. 


\section{APPENDIX}

\section{LIST OF ABBREVIATIONS}

4-HNE: 4-hydroxy-2-nonenal

Acrolein: propen-2 al

AP-1: Activator protein 1

Aldh: aldehyde dehydrogenases

LPO: Lipid peroxidation

MDA: malondialdehyde

WT: wild type

ORF: open reading frame

CYP: Cytochrome P450

ROS: reactive oxygen species

MTT: 3-(4,5-dimethylthiazol-2-yl)-2, 5-diphenyltetrazolium bromide

SDS-PAGE: sodium dodecyl sulfate-polyacrylamide gel electrophoresis

AKR: aldoketoreductase

Nrf2: Nuclear factor-E2 related factor 2

ARE: Antioxidant response element

BHA: butylated hydroxyanisole

tBHQ: tert-butylhydroquinone

CAR: Constitutive androstane receptor

PB: Phenobabitals 
SFN: Sulforaphane

GSH: Glutathione

GST: Glutathione-S transferase

ADH: Alcohol dehydrogenase

GS-HNE: glutathione conjugate of 4-HNE

Keap-1: Ketch-like ECH-associated protein 1

$\mathrm{NAD}(\mathrm{P})+$ : Nicotine adenine dinucleotide phosphate, oxidized form

NF-kB: nuclear factor kappa B

PCR: Polymerase chain reaction

QRT-PCR: Quantitative real time PCR

RXR: retinoid X receptor

RAR: retinoic acid receptor

TPA: 12-O-tetradecanoyl-phorbol-13 acetate

TRE: TPA response element 


\section{CURRICULUM VITAE}

\section{Ngome L Makia, B.Sc., M.S.}

\section{OFFICE ADDRESS}

Department of Biochemistry and Molecular Biology

University of Louisville School of Medicine

580 S. Preston St, Louisville

Delia Baxter Bldg, Room 107

Louisville, KY 40202

Work: (502) 8527947

nlmaki01@gwise.louisville.edu

\section{HOME ADDRESS}

6401 Fern Valley Ct, Apt 4 Louisville, KY 40219

Cell: (502) 6447179

\section{EDUCATION}

07/2006 - Present University of Louisville, Louisville, KY

Ph.D candidate, anticipated graduation date: August 2011

Discipline of study: Biochemistry \& Molecular Biology

09/2003 - 12/2004 University of Nottingham, Nottingham, United Kingdom

M.Sc (Distinction), December 2004

Discipline of study: Biomolecular Technology

09/1997 - 12/2000 University of Buea, Buea, Cameroon

B.Sc (First Class Honors; GPA 3.71), December 2000

Major: Biochemistry, Minor: Medical Laboratory Technology

\section{SCIENTIFIC/PROFESSIONAL EXPERIENCE}

2006-Present

Graduate Research Assistant, Department of Biochemistry \& Molecular Biology, University of Louisville, Louisville, KY. Dissertation project title: Cytotoxicity and cellular response to $\alpha$, $\beta$-unsaturated aldehydes. The role of aldehyde dehydrogenases Principal Investigator: Russell A Prough, Ph.D.

\section{Research experience:}

- Design and perform experiments for my research project, interpret data and trouble shoot experiments.

- Use C57BL6 and Nrf2 ${ }^{-/ 2}$ mice fed AIN-76 diet or diet containing BHA for enzyme assay, western blot analysis, RNA isolation, quantitative real-time PCR, isolation of cytoplasmic and nuclear protein, gene expression studies. 
- Maintain culture of the human (HepG2) and mouse (Hepa-1c1c7) hepatoma cell lines for transient transfection experiments with Aldh1a1 promoter luciferase constructs.

- $\quad$ Luciferase and $\beta$-galactosidase activity assay, Electrophoretic Mobility Shift Assay and Chromatin Immunoprecipitation Assay

- Clone and express mouse Aldh1a1, Aldh2 and Aldh3a1 in E coli with N-terminal his-tag; purify enzymes using Ni-NTA resin and kinetic characterization of Aldhs

- Gene cloning technique, site directed mutagenesis and agarose gel electrophoresis

- Participate and present in weekly laboratory meetings.

- Grant and manuscript writing.

01/2005-07/2006 Research Assistant, School of Biosciences, University of Nottingham, United Kingdom

Principal Investigator: Stephen E. Harding, Ph.D.

\section{Research experience:}

- Macromolecular characterization techniques: AUC, SEC-MALLS, DLS, viscometry, SDS-PAGE, IEF, ELISA and ESI-MS/MS.

- Computer software for sedimentation velocity and equilibrium analysis: SVEDBERG, DCDT, SEDNTERP, PERKINS, ELLIPSI, NON-LIN and MSTAR.

- $\quad$ Experience in 3-D molecular imaging with Rasmol

06/2004 -09/2004 Industrial Placement in Partial Fulfillment of an M.Sc in Biomolecular Technology at Cambridge Antibody Technology, Product Characterisation Unit, Franklin Building, Granta Park, Cambridge, CB1 6GH, England.

Thesis Title: Physicochemical characterization of Human IgG4 expressed using different mammalian cell lines (CHO, NSO, HEK293) by ESI-MS/MS, IEF and SDS-PAGE:_Principal Investigators: Sandrine Mulot, Ph.D.

\section{Research experience:}

- Isolate human IgG4 antibodies from three mammalian cell lines, NS0, CHO and HEK293. Analysis after DTT treatment and alkylation with Iodoacetamide, followed by tryptic digestion and ESI-MS/MS

- Compare heterogeneity in the antibodies isolated from various cell lines using IEF and SDS-PAGE

- Daily laboratory maintenance.

- Participate and present in weekly Journal club meetings.

- Thesis writing.

07/2001-09/2002

Research Assistant: Diagnosis of HIV using screening and confirmatory tests (ELISA). Virus Immunlogy Unit, Faculty of Medicine and Biomedical Sciences, University of Yaounde 1-Cameroon. 


\section{SUMMARY OF Ph.D DISSERTATION RESEARCH}

Reactive lipid aldehydes are implicated in the pathogenesis of various oxidative stress-mediated diseases, including the transition from hepatic steatosis to non-alcoholic steatohepatitis (NASH). The role of Aldehyde dehydrogenase (Aldh) isozymes in detoxification of lipid aldehydes, such as acrolein and HNE in mice liver is highly controversial. The first part of my thesis was to define which hepatic Aldh isoforms plays a major role in detoxification of lipid-derived aldehydes in mice liver by enzyme kinetics and in vitro knockdown experiment. Kinetic studies revealed that Aldhlal exhibits higher affinity for acrolein and HNE compared to Aldh2 and Aldh3a1. We also demonstrated that knockdown of Aldhlal expression by siRNA caused lipid-derived cells to be more sensitive to acrolein-induced cell death, and resulted in increased accumulation of acrolein-protein adducts and activation of caspase 3 . These results indicate that Aldhlal plays a major role in cellular defense against oxidative damage induced by reactive lipid aldehydes in mouse liver. We also noted that hepatic Aldh1al mRNA levels were significantly increased $(\approx 3$-fold) in acrolein-fed mice compared to control. In addition, hepatic cytosolic ALDH activity was induced by acrolein when 1 mM NAD ${ }^{+}$was used as cofactor, suggesting an Aldhla1-protective mechanism against acrolein toxicity in mice liver. Thus, mechanisms to induce Aldhlal gene expression may provide a useful rationale for therapeutic protection against oxidative stress-induced pathologies.

The second part of my thesis was to decipher the mechanisms of electrophileinduced transcription of Aldh1a1. We analyzed the mRNA levels of hepatic Aldhlal in C57BL6 (WT) and Nrf2 ${ }^{-/-}$mice exposed to butylated hydroxyanisole (BHA) by qRT$\mathrm{PCR}$. In vivo, BHA is demethylated to tert-butylhydroquinone (tBHQ), which is known to undergo redox cycling to tert-butylquinone (tBQ) generating ROS. BHA exposure resulted in $\approx 2$-fold increase in mRNA levels of Aldhlal in WT and $\mathrm{Nrf}^{-/-}$mice compared to control of the same genotype, while the induction of Nrf2 target genes, such as Nqo-1, Ho- 1 and Gstm1 upon BHA administration was significantly reduced in $\mathrm{Nrf}^{-/-}$ mice. The mRNA and protein levels of the AP-1 genes (c-Jun and c-fos) were significantly induced by BHA treatment in WT mice. Transient transfection experiments were conducted in HepG2 cells with Aldhlal 5'-flanking luciferase reporter vectors. While co-transfection with $\mathrm{Nrf} 2$ expression plasmid had no effect, over-expression of cJun resulted in $\approx 4$-fold induction in Aldh1a1 transcriptional activity. We proposed that electrophile-induced transcription of hepatic Aldh1al is mediated by c-Jun/AP-1. Overexpression of a dominant negative c-Jun (TAM67) protein in HepG2, treatment with 50 $\mu \mathrm{M}$ JNK (SP600125) or MEK1 (PD98059) inhibitors significantly abrogate the electrophile-mediated transcription of Aldh1al. We further showed that c-Jun induces Aldhlal promoter activity as a homodimer and not as c-Jun/c-Fos heterodimer. Deletion and mutagenesis studies established that two AP-1 sites at position -758 and -1069 relative to Aldh1a1 transcription start site mediate c-Jun-induced transcription of Aldh1a1. We further confirmed that electrophiles promote the binding of c-Jun to the proximal AP-1 site by electrophoretic mobility shift assay (EMSA) and ChIP assay. Taken together, these data suggest that Aldhlal may be a novel therapeutic target for preventing the transition from fatty liver to NASH. 


\section{PUBLICATIONS}

1. Makia, N.L., Bojang, P., Falkner, K.C., Conklin, D.J., Prough, R.A. Murine hepatic aldehyde dehydrogenase lal is a major contributor to oxidation of aldehydes formed by lipid peroxidation. Chemico-Biological Interactions, May 2011, Vol. 191, No, 1-3.

2. I. Amunom, N. Makia, D.J. Conklin, A. Bhatnagar, S. Suripureddi, J.A. Goldstein and R.A Prough. AP-1 Regulation of Cyp2c29 in liver and vascular tissues of the mouse". Molecular Pharmacology, In Revision (2011).

3. Makia, N.L., Amunom, I., Falkner, K.C., Conklin, D.J., Prough, R.A. Role of AP-1 transcription factor in the induction of Aldhlal gene by electrophiles. Molecular Pharmacology, Manuscript in Preparation (2011).

\section{ABSTRACTS}

1. N.L. Makia, K.C. Falkner, D.J. Conklin, A. Bhatnagar, and R.A. Prough. Gene Expression of Aldehyde Dehydrogenases Involves NF-E2-related factor-2 (Nrf2). Drug Metabolism Reviews, Vol. 40, No. s3: 234 (2008).

2. N.L. Makia, K.C. Falkner, D.J. Conklin, A. Bhatnagar, and R.A. Prough. Role of c-Jun/AP-1 in oxidative stress-mediated induction of murine Aldhlal gene. Drug Metabolism Reviews, Vol. 41, No. s3: 105 (2009)

3. N. Makia, I. Amunom, D.J. Conklin, A. Bhatnagar, and R.A. Prough. Adaptive response to reactive aldehydes in mouse liver: Role of aldehyde dehydrogenases. Research Louisville, 2009

4. N. Makia, I. Amunom, D.J. Conklin, A. Bhatnagar, and R.A. Prough. Adaptive response to reactive aldehydes in mouse liver: Role of aldehyde dehydrogenases. 15th International Meeting; Enzymology and Molecular Biology of Carbonyl metabolism, Lexington, KY, July 6-11, 2010.

5. N.L. Makia, K.C. Falkner, D.J. Conklin, and R.A. Prough. Molecular regulation of aldehyde dehydrogenase 1al by electrophiles: Role of AP-1 transcription factors. 17th North American Meeting of the International Society for the Study of Xenobiotics, Atlanta, GA, October 16-20, 2011 , Abstract 25781.

\section{FUTURE AREA OF RESEARCH INTEREST}

Hepatology research, liver toxicity by environmental aldehydes, mechanisms of cytotoxicity by reactive lipid aldehydes, redox-sensitive transcription factors, such as $\mathrm{Nrf} 2, \mathrm{AP}-1$ and NF-kB, drug-metabolizing enzymes, transporters and their regulation.

\section{TRAINING/MENTORING EXPERIENCE}

Summer 2007 Supervised and trained Zack Simpson (2nd year Medical student) on the cloning and expression of Aldh1a1, Aldh2 and Aldh3a1 in E coli, and their purification and characterization. Assess the purity of protein by SDS-page gel electrophoresis.

Summer 2009 Supervised and trained Luz E Guevara (Undergraduate student, Montclair State University) on the cloning, expression, purification and characterization of Aldh1a7. Assess the purity of protein by SDS-page gel electrophoresis.

Summer 2010 Supervised and trained Sara Heintzman (2nd year Medical student) on the molecular cloning and kinetic characterization of ALDH2; the effect of knockdown of 
Aldh2 by siRNA in Hepa- $1 \mathrm{c} 1 \mathrm{c} 7$ on lipid aldehyde-induced toxicity. SDS-PAGE electrophoresis and preparation of protein lysates for western blot analysis.

\section{LEADERSHIP SKILLS}

$2009-2010$ Vice-President Biochemistry student body. Department of Biochemistry and Molecular Biology, University of Louisville, KY.

\section{PROFESSIONAL SOCIETIES}

- International Society for the Study of Xenobiotics (ISSX)

- Society of Carbonyl Enzymology and Molecular Biology of Carbonyl

- Golden Key International Honor Society

\section{HONORS \& AWARDS}

2008

2008

2006-2007

$12 / 2004$

2003-2004

2000

$1997-2000$
CGEMM Travel Award to attend the 15th North American Meeting of the International Society for the Study of Xenobiotics, San Diego, CA, School of Medicine Travel Award IPIBS Fellowship, University of Louisville, School of Medicine Graduation with Distinction, University of Nottingham, United Kingdom Commonwealth Scholarship award to study for a masters degree in Biomolecular Technology, University of Nottingham, UK University of Buea Biochemistry Student Association (BUBSA) prizes for outstanding Biochemistry students (2000), Buea, Cameroon Dean's list. Faculty of Science-University of Buea, Buea, Cameroon

\section{REFERENCES}

\section{Russell A. Prough, Ph.D}

Vice Dean for Research

Preston Pope Joyes Endowed Chair in Biochemical Research

Department of Biochemistry and Molecular Biology

School of Medicine, University of Louisville

Room 102B, Delia Baxter Bldg

580 S. Preston St

Louisville, KY 40202-1756

Phone Number: (502)852-7249

FAX : (502) 852-2552

Email: russ.prough@louisville.edu

\section{Kenneth S. Ramos, Ph.D}

Director, NIEHS Center for Environmental Genomics and Integrative Biology

Professor of Biochemistry and Molecular Biology

University of Louisville

580 S. Preston St., Baxter II, Room 221

Louisville, KY 40202 
Phone Number: (502)852-7207

Email: ksramo01@1ouisville.edu

Daniel J. Conklin, Ph.D.

Associate Professor of Molecular Cardiology

Division of Cardiovascular Medicine

Department of Medicine

Delia Baxter, 421C

University of Louisville

Louisville, KY 40202

Phone Number: (502)852-5836

Email: $\underline{\text { d0conk01@,louisville.edu }}$ 